\title{
Structure Formation and Collective Behavior of Dipolar Active Particles
}

\author{
Dissertation \\ for the award of the degree \\ "Doctor rerum naturalium" \\ of the Georg-August-Universität Göttingen \\ within the doctoral program \\ Physics of Biological and Complex Systems \\ of the Georg-August University School of Science (GAUSS) \\ submitted by \\ Vitali Telezki \\ from Shambyl (Kazakhstan)
}

Göttingen 2021 


\section{Thesis Committee}

\section{Prof. Dr. Stefan Klumpp}

Institute for the Dynamics of Complex Systems

University of Göttingen

Prof. Dr. Marcus Müller

Institute for Theoretical Physics

University of Göttingen

Prof. Dr. Karen Alim

Biological Physics and Morphogenesis

Technical University of Munich

\section{Members of the Examination BoARD}

Referee: Prof. Dr. Stefan Klumpp

Institute for the Dynamics of Complex Systems University of Göttingen

$2^{\text {nd }}$ Referee: Prof. Dr. Marcus Müller

Institute for Theoretical Physics

University of Göttingen

Prof. Dr. Karen Alim

Biological Physics and Morphogenesis

Technical University of Munich

Prof. Dr. Jörg Enderlein

Third Institute of Physics - Biophysics

University of Göttingen

Dr. Aljaž Godec

Mathematical bioPhysics Group

Max Planck Institute for Biophysical Chemistry

Prof. Dr. Peter Sollich

Institute for Theoretical Physics

University of Göttingen

Date of oral examination: 08.12 .2021 


\section{Zusammenfassung}

Dipolare aktive Teilchen gehören zu einer Klasse von selbst angetriebenen Teilchen biologischen oder künstlichen Ursprungs. Diese Teilchen verfügen über ein internes, typischerweise magnetisches Dipolmoment. Die daraus entstehenden dipolaren Teilchen-Teilchen Wechselwirkungen führen in Kombination mit dem aktiven Antrieb zu komplexem kollektivem Verhalten in Systemen solcher Teilchen.

In der vorliegenden Arbeit verwenden wir Brownsche Dynamik-Simulationen, um die Vielfalt der dynamischen Strukturbildung in solchen System zu untersuchen. Dabei ist diese Arbeit in vier Hauptforschungsthemen gegliedert. Zunächst untersuchen wir Strukturbildung in kleinen Systemen von dipolaren aktiven Teilchen. Hier beobachten wir, dass sich vor allem Ringe und Ketten bilden. Diese Strukturen sind hauptsächlich abhängig von der Aktivität und der Magnetisierung der Teilchen. Zudem beeinflussen auch Wechselwirkungen mit Wänden im System die Strukturbildung. Im nächsten Schritt gehen wir zu großen Systemen dipolarer aktiver Teilchen über. Wir zeigen, dass Aktivität eine entscheidende Rolle bei dem emergenten Verhalten spielt. Wir klassifizieren das beobachtete kollektive Verhalten und fassen die Ergebnisse in Zustandsdiagrammen zusammen. Als drittes untersuchen wir den Einfluss eines konstanten homogenen externen Magnetfeldes auf die Strukturbildung. Dabei beobachten wir, dass Bildung von Bändern durch starke externe Magnetfelder unterdrückt wird und sich Teilchen stattdessen in säulenartige Strukturen organisieren. Außerdem stoßen wir auf einen bisher nicht charakterisierten vorübergehenden Zustand von oszillierenden Ketten aktiver dipolarer Teilchen. Wir gehen davon aus, dass diese Oszillationen durch BucklingInstabilitäten verursacht werden. Zuletzt führen wir ein zeitabhängiges externes Magnetfeld ein und untersuchen die dadurch angetriebene Dynamik der Strukturbildung.

Diese Arbeit zeigt, wie dipolare Wechselwirkungen, der Einfluss externer magnetischer Felder oder Wechselwirkungen mit Wänden des Systems, das bereits bekannte, vielfältige komplexe kollektive Verhalten in Systemen aktiver Teilchen erweitern und bereichern können.

Stichwörter: aktive Brownsche Teilchen, Brownsche Dynamik-Simulationen, dipolare Teilchen, Emergenz, externes Magnetfeld, kollektives Verhalten, Nichtgleichgewichtssystem, Simulationen, Strukturbildung, theoretische Physik, Wandwechselwirkungen, zeitabhängiges Magnetfeld, Zustandsdiagramm. 


\section{Abstract}

Dipolar active particles describe a class of self-propelled, biological or artificial particles. These particles are equipped with an internal, typically magnetic, dipole moment. The combination of dipolar particle-particle interactions and activity leads to emerging complex collective behavior in systems of such particles.

In this thesis, we use Brownian dynamics simulations to explore and characterize the plethora of structural dynamics and pattern formation in systems of dipolar active particles. This study can be divided into four parts. First, we focus on structure formation in small systems. Here, we mainly observe chain and ring formation and characterize how activity and spatial confinement affects these structures. Second, we move to large systems of dipolar active particles, classify the collective patterns we observed and summarize our results in diagrams of states. We show that activity plays a crucial role in the emergent collective behavior in systems of dipolar active particles. Third, we investigate the effect a constant homogeneous external magnetic field has on collective dynamics of the system. We observe that band formation is suppressed by strong external magnetic fields and columnar structures form. In addition, we notice a previously not characterized transient state of oscillating chains of active dipolar particles. We hypothesize that these oscillations are caused by buckling instabilities. Finally, we introduce a time dependent external magnetic field and study the dynamics of structure formation driven by that field.

This thesis demonstrates how dipolar interactions, external magnetic fields, or confinement, can further add to the rich complex collective behavior in systems of active particles.

Keywords: active Brownian particles, Brownian dynamics simulations, confinement, collective behavior, diagram of states, dipolar particles, external magnetic field, non-equilibrium physics, numerical simulations, order parameter, pattern formation, structure formation, theoretical physics, time dependent external magnetic field. 


\section{Contents}

\begin{tabular}{ll}
\hline 1. Introduction & 1
\end{tabular}

1.1. Outline. . . . . . . . . . . . . . . . . . . 5 5

\begin{tabular}{ll}
\hline 2. Methods & 7
\end{tabular}

2.1. Equations of motion $\ldots \ldots \ldots \ldots \ldots \ldots$

2.2. Identifying clusters and structures of polymers . . . . . . . . . 13

2.2.1. Dynamics of chains and rings . . . . . . . . . . 15

2.3. Global order parameters . . . . . . . . . . . . . . . . 16

3. Computational details 19

3.1. Simulation method . . . . . . . . . . . . . . . . . . . 19

\begin{tabular}{|l|}
\hline 4. Structure formation in small systems \\
\hline
\end{tabular}

4.1. Assembly of chain and ring structures . . . . . . . . . . . 26

4.1.1. Classification of structures . . . . . . . . . . . . . . 30

4.2. Influence of confinement on structure formation . . . . . . . . . 33

4.2.1. Influence of density in confinement . . . . . . . . . 40

4.3. Comparison to analytical solution . . . . . . . . . . . 45 
5. Collective dynamics in large systems without external field 50

5.1. Low density $\Phi=0.13$. . . . . . . . . . . . . . . . . . . . . . 51

5.2. Intermediate density $\Phi=0.23 \ldots \ldots \ldots \ldots$

5.3. High density $\Phi=0.57 \ldots \ldots \ldots$. . . . . . . . . . . 63

6. Influence of constant external magnetic field 70

6.1. Low density $\Phi=0.13$. . . . . . . . . . . . . . . . . . 71

6.2. Intermediate density $\Phi=0.23 \ldots \ldots \ldots$. . . . . . . . 78

6.2.1. Columnar structures . . . . . . . . . . . . . . . . . 79

6.3. High density $\Phi=0.57$. . . . . . . . . . . . . . . . . . . . . 83

7. Collective dynamics in time-dependent external field 86

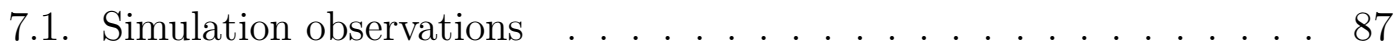

7.2. Coercive force and relative remnant magnetization . . . . . . . . . . 89

7.3. Dynamic structure formation . . . . . . . . . . . . . . . 91

$\begin{array}{ll}\text { 8. Conclusions } & 94\end{array}$

\begin{tabular}{lr}
\hline A. Simulation details & 100
\end{tabular} 


\section{Nomenclature}

\section{Symbols}

Vectors are denoted by small, bold italic letters $\boldsymbol{v}$. The superscript 'hat' denotes a unit vector $\hat{\boldsymbol{e}}$.

\begin{tabular}{lll} 
symbol & meaning & unit \\
\hline$B$ & external magnetic field strength & $\mathrm{B}_{0}$ \\
$\beta$ & ramping rate & $\mathrm{B}_{0} \sigma / \mathrm{D}_{\mathrm{T}}$ \\
$\Delta t$ & time step & $\mathrm{D}_{\mathrm{T}} / \sigma$ \\
$\epsilon$ & cut-off distance cluster & $\sigma$ \\
$L$ & side lengths simulation box & $\sigma$ \\
$\mu$ & magnetic moment & $\epsilon / \mathrm{B}_{0}$ \\
$N$ & particle number & 1 \\
$\Phi$ & filling fraction & 1 \\
$R$ & radius of circular confinement & $\sigma$ \\
$t$ & time & $\mathrm{D}_{\mathrm{T}} / \sigma$ \\
$T$ & temperature & $\epsilon / \mathrm{k}_{\mathrm{B}}$ \\
$v_{0}$ & self-propulsion speed & $D_{T} / \sigma$
\end{tabular}


Nomenclature

\section{Abbreviations}

abbreviation meaning

BD Brownian dynamics

$\operatorname{cog} \quad$ center of geometry

MD molecular dynamics

MIPS motility induced phase separation

MSD mean-squared displacement

pbc periodic boundary conditions

WCA Weeks-Chandler-Andersen

viii 


\section{Introduction}

Dipolar active particles, or dipolar swimmers, describe a class of self-propelled, biological or artificial particles. These particles are equipped with an internal, typically magnetic, dipole moment. In general, self-propelled particles constantly convert energy from their environment into directed motion [1, 2]. Directed motion is crucial for the survival of living organisms, ranging from bacteria over animals to humans. It is essential in the search for food, to escape predators or unwanted environmental conditions.

Active particles in large ensembles do not only interact with their environment, but also with other particles. Because of various interaction forces, systems consisting of many similar units of active particles are known to exhibit rich collective behavior such as self-organization, pattern formation and swarming [3] [5]. Everyday examples of complex collective behavior are a school of fish or a flock of birds [6 9]. But also particles on the micro-scale, so called microswimmers, like bacteria or microalgae can exhibit rich collective behavior. Here, models have shown that the details of the propulsion mechanism are largely not relevant $10-13$.

The physics involved in swimming on microscopic length scales is vastly different from swimming on macroscopic length scales known to humans for two reasons 14 17]. First, particles on the micro-scale are subjected to noise: solvent molecules 


\section{Introduction}

constantly collide with the microscopic particle and cause random translational and rotational motion. This motion is known as Brownian motion and is the reason for diffusive behavior of passive mircometer-sized particles [18, 19]. Microswimmers use active self-propulsion to overcome diffusion. Second, at small length scales viscous forces dominate over inertial forces when swimming in water. To achieve directed motion in this low Reynolds number regime, the swimming motion of microscopic particles has to be reciprocal but asymmetric under time-reversal [20].

Microswimmers have been a particular focus of research because of their possible biomedical applications 21, 22. From an application point of view, the desire for remote control of active particles is high. In that respect, magnetic active particles are an ideal candidate because they can be controlled non-invasively by an external magnetic field. One example for a biological dipolar swimmer are magnetotactic bacteria (MTB) 23 28. Fig. 1.1 shows two examples of MTB. These bacteria align with external magnetic fields because of the magnetosome chain, a chain of ironoxide or sulfite nanocrystals located inside their cell bodies. Recent studies have demonstrated concepts where magnetotactic bacteria have been functionalized to transport drugs into tumor regions [29] or dense biofilms [30]. Here, it has been shown that externally guided microswimmers can increase the efficacy in the region of interest dramatically.

Inspired by these biohybrids, artificial magnetic microswimmers have been developed [14, 22, 31]. Typically, these artificial microrobots realize self-propulsion via magnetic actuation through an external time-dependent magnetic field [21] or through an external electric field that induces magnetic interactions between particles [32]. Here, assembly into structures can be dictated by manipulating the external field. One example are magnetic Janus particles that can self-assemble 

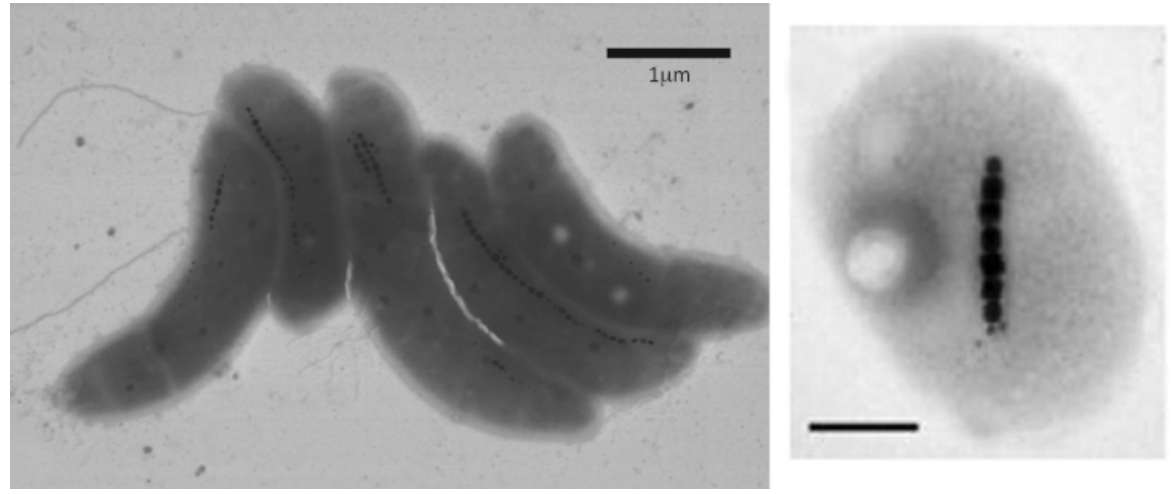

Figure 1.1.: Transmission electron microscopy images showing two examples for magnetotactic bacteria. Left Magnetospirillum gryphiswaledense MSR-1 strain (reprinted from ref. 27] under the creative commons CC-BY 4.0 license). Right SS-1 strain (reprinted from ref. [26] under the creative commons CC-BY-NC-ND 3.0 license). Black dots inside the cell bodies are the magnetosome chains.

into various structures which can be controlled by external magnetic fields 33 , 34]. Other self-propulsion mechanisms rely on chemical reactions [35, 36] or thermophoresis [37].

While the dipole moment of these active particles is mostly used for guidance, dipolar particle-particle interactions are inevitably introduced. These long range and strongly direction dependent interactions may lead to previously unknown spatial patterns or new forms of collective behavior 38 40].

Structure formation in passive systems of dipolar particles has been studied in the past both theoretically and in experiments. Calculations of the configurational energy in two dimensions have shown that the energetically favorable configuration depends on the number of particles $N$. Systems with small particle number $N$ are known to adopt chains $(N<3)$, rings $(3<N \leq 17)$ or more complex, concentric multi-shell hexagonal patterns $(N>17)$ as favorable configurations [41-44]. In fact, it has been argued that chain aggregation suppresses the usually observed vapor-liquid phase transition in systems of dipolar particles [45 48]. Mo- 


\section{Introduction}

tivated by observations in ferrofluids [49], temperature dependence of chain and ring structures in passive systems has been investigated and identified as ground state structures $50-52]$. Furthermore, an analytical expression for the contributions to the systems' free energy by chains and rings has been derived [53]. Systems with larger particle number $N$ of passive dipolar particles, as realized in ferrofluids, show more complex structures like lanes, complex networks with branches and percolation $38,54,57$.

Ferrofluids that are subject to external magnetic fields have been studied in the past in the field of magnetorheology. Here, mechanical properties such as the viscosity of the system can be tuned over several orders of magnitude by the external magnetic field. This remarkable property makes such systems very good candidates for applications in mechanical systems like dampers, clutches or breaks [58]. Experiments and numerical simulations of dipolar particles in an external field 59 61] have demonstrated that structures like equally spaced, columnar ordered chains and bands are induced by the external magnetic field $[57,62,63]$. These complex structures give rise to the mechanical properties of ferrofluids [64, 65].

In some studies, the effect of time-dependent external magnetic fields in ferrofluids was investigated too. Here, time dependency was typically realized by a rotating external magnetic field [66, 67]. These studies focused on the elastic properties of chains, similar to [44]. Time-dependent external magnetic fields may introduce hysteresis [68], as seen in other magnetic systems [42, 69].

In recent years, also active systems of dipolar particles were studied by several groups [40]. Of particular interest was the question, how hydrodynamics affect structures and clusters of dipolar particles 70,71 . In other studies the effect of dipolar coupling and activity on cluster formation has been investigated and 


\subsection{Outline}

summarized in a phase diagram $[72]$. Brownian dynamics simulations have shown that motility induced phase separation (MIPS) is generally suppressed by dipolar interactions 73$]$. MIPS is expected to occur in systems of active particles without attractive interactions when the average density exceeds the critical density $\Phi_{\mathrm{MIPS}}=0.28[74$ in two dimensions. Many factors can influence the exact value of this critical density, like systems size and finite size effects $[75$ or softness of the particle-particle interaction potential [76]. In addition, mixtures of passive dipolar particles in a bath of active particles have been investigated without [77] and with the presence of an external magnetic field 78 .

Most of these studies have focused on systems in spatially homogeneous environments. Yet, microswimmers often interact with interfacial barriers present in their natural environment or are confined by complex experimental setups like microfluidic devices. It has been shown that confinement can affect the motion of microswimmers either via steric and/or hydrodynamic interactions to different degrees 7985$]$, sometimes leading to novel dynamics and pattern formation 86 88.

\subsection{Outline}

Systems of active dipolar particles are known to exhibit rich collective dynamics. The aim of this thesis is to expand the current understanding of structure formation and collective behavior of systems of active dipolar particles, such as magnetotactic bacteria or artificial magnetic swimmers under various conditions. To that end, we use a coarse grained model for dipolar active particles. The model and its corresponding equations of motion are introduced in chapter 2 , The equations 


\section{Introduction}

of motion are solved numerically in two dimensions. Computational details of the simulations can be found in chapter 3. The results of these simulations are presented and discussed in chapter 4 to chapter 7 . The main research questions addressed in this thesis are divided into four parts.

First, we investigate structure formation of dipolar active particles in systems with small particle number $N=36$ and low average density $\Phi$ in chapter 4 . Here, we ask what structures like chains and rings can emerge and how are these structures affected by activity and magnetic interactions. In addition, we introduce confining walls to the system and analyze how structure formation is influenced by the geometry and by the interactions between the active particles with these confining walls.

Second, we increase the number of particles to $N=1156$ and study the collective behavior of active dipolar particles in bulk and how it is affected by the systems' density $\Phi$ in chapter 5. We quantify the observed collective behavior by introducing three order parameters and summarize the results in diagrams of states.

Third, we add a constant homogeneous external magnetic field to the bulk system of active dipolar particles and study the effect of this field on the collective motion of the system in chapter 6. We are particularly interested in emerging columnar clusters that are induced by the external magnetic fields.

Fourth, we change the constant external magnetic field into a time-dependent one in chapter 7 . The time dependency of the external field is modulated by a triangular function with a constant ramping rate. Here, we investigate how this ramping rate affects assembly, disassembly and reassembly of structures for different motility and magnetic interactions of dipolar active particles

Finally, we summarize our results and draw general conclusions in chapter 8 . 


\section{Methods}

In this chapter, we present the coarse grained model that we used to investigate collective dynamics of active dipolar particles throughout this thesis. We will start by introducing the equations of motion. Furthermore, we define in this chapter the observables and order parameters that we used to quantify our observations with in this thesis.

\subsection{Equations of motion}

To model the dynamics of dipolar swimmers, we considered active Brownian spheres that have a permanent dipole moment $\boldsymbol{\mu}_{i}=\mu \hat{\boldsymbol{e}}_{i}$ with the magnetic strength $\mu$ at the particle center. Here and in the following, the superscript 'hat' denotes a unit vector. As shown in Figure 2.1, the magnetic moment (red arrow) is aligned with the orientation of the particle $\hat{\boldsymbol{e}}_{i}$, which defines the direction of self-propulsion with speed $v_{0}$. Two dipolar particles with the orientations $\hat{\boldsymbol{e}}_{i}$ and $\hat{\boldsymbol{e}}_{j}$, separated by the distance $r_{i j}=\left|\boldsymbol{r}_{i j}\right|$ interact with each other via steric interactions and dipolar interactions. Steric interactions are modeled by a Weeks-Chandler-Andersen (WCA) potential [89], which is the shifted Lennard-Jones potential truncated at 


\section{Methods}

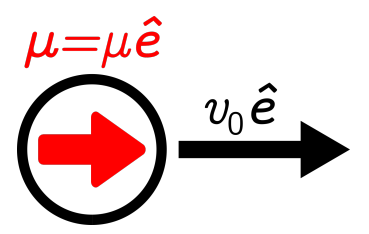

Figure 2.1.: Schematic representation of a single dipolar particle with magnetic moment (red arrow) and self propulsion velocity (black arrow) aligned with the orientation of the particle.

$\sqrt[6]{2} \sigma$, where $\sigma$ is the particle diameter

$$
U^{\mathrm{WCA}}\left(r_{i j}\right)= \begin{cases}4 \epsilon\left[\left(\frac{\sigma}{r_{i j}}\right)^{12}-\left(\frac{\sigma}{r_{i j}}\right)^{6}\right]+\epsilon & \text { if } r_{i j}<\sqrt[6]{2} \sigma \\ 0 & \text { if } r_{i j} \geq \sqrt[6]{2} \sigma\end{cases}
$$

Dipolar interactions between two particles with magnetic moments $\boldsymbol{\mu}_{i}=\mu_{i} \hat{\boldsymbol{e}}_{i}$ and $\boldsymbol{\mu}_{j}=\mu_{j} \hat{\boldsymbol{e}}_{j}$ are modeled by a pairwise dipole potential

$$
U^{\mathrm{dd}}\left(\boldsymbol{r}_{i j}, \boldsymbol{\mu}_{i}, \boldsymbol{\mu}_{j}\right)=\frac{\mu_{0}}{4 \pi r_{i j}^{5}}\left[-3\left(\boldsymbol{\mu}_{i} \cdot \boldsymbol{r}_{i j}\right)\left(\boldsymbol{r}_{i j} \cdot \boldsymbol{\mu}_{j}\right)+r_{i j}^{2} \boldsymbol{\mu}_{i} \cdot \boldsymbol{\mu}_{j}\right]
$$

with the vacuum permeability $\mu_{0}$. One characteristic feature of this potential is its long range, as it decays as $r^{-3}$. In addition, dipolar interactions are strongly anisotropic and favor certain spatial configurations of two particles. Four typical configurations of two particles with magnetic strength $\mu_{i}=\mu_{j}=\mu$, separated by distance $\left|\boldsymbol{r}_{i j}\right|=r_{i j}=\sigma$ are shown in Figure 2.2. Here, a head-tail configuration is energetically favorable. Both features, the long range and anisotropy of interactions, are crucial for the formation of complex structures and collective behavior of systems with active dipolar particles. We study structure formation in small systems in chapter 4 and emerging collective behavior in chapter 5 .

The dynamics of a dipolar active particle $i$ with position $\boldsymbol{r}_{i}$ and orientation $\hat{\boldsymbol{e}}_{i}$ 


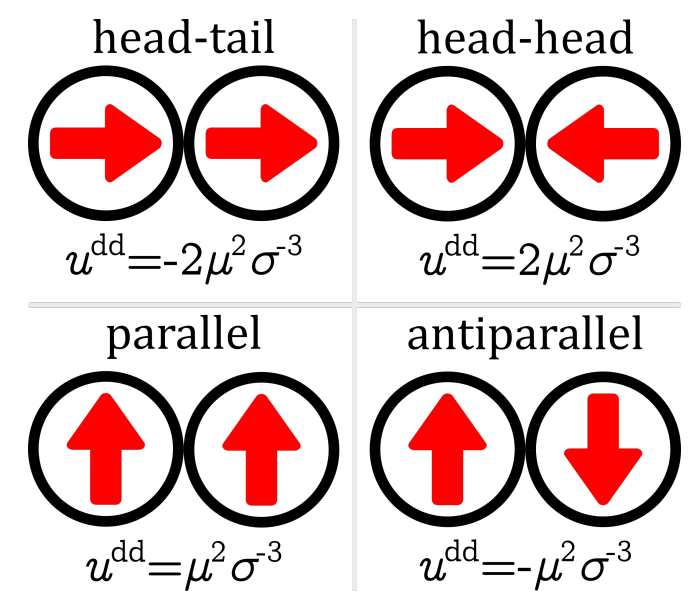

Figure 2.2.: Schematic showing four typical configurations and their corresponding potential energies in reduced units of two dipolar particles separated by distance $\sigma$. The orientation of the magnetic moments are indicated by red arrows.

are given by the following Langevin equations

$$
\begin{aligned}
& \dot{\boldsymbol{r}}_{i}=v_{0} \hat{\boldsymbol{e}}_{i}+\frac{1}{\gamma_{\mathrm{T}}} \boldsymbol{F}_{i}+\sqrt{2 D_{\mathrm{T}}} \boldsymbol{\xi}_{i}^{\mathrm{T}} \\
& \dot{\hat{\boldsymbol{e}}}_{i}=\frac{1}{\gamma_{\mathrm{R}}} \boldsymbol{\tau}_{i} \times \hat{\boldsymbol{e}}_{i}+\sqrt{2 D_{\mathrm{R}}} \boldsymbol{\xi}_{i}^{\mathrm{R}} \times \hat{\boldsymbol{e}}_{i},
\end{aligned}
$$

where $\gamma_{\mathrm{T}}$ and $\gamma_{\mathrm{R}}$ are the translational and rotational drag coefficients, $\boldsymbol{\xi}_{i}^{\mathrm{T}}$ is the translational stochastic force, and $\boldsymbol{\xi}_{i}^{\mathrm{R}}$ is the rotational stochastic torque. These stochastic terms model the particles interactions with its surrounding fluid. Both are described by Gaussian white noise with zero mean

$$
\begin{aligned}
\left\langle\boldsymbol{\xi}^{\mathrm{T}, \mathrm{R}}\right\rangle & =0 \\
\left\langle\boldsymbol{\xi}^{\mathrm{T}, \mathrm{R}}(t) \cdot \boldsymbol{\xi}^{\mathrm{T}, \mathrm{R}}\left(t^{\prime}\right)\right\rangle & =\mathbf{1} \delta\left(t-t^{\prime}\right) .
\end{aligned}
$$

$D_{\mathrm{T}}$ and $D_{\mathrm{R}}$ are the corresponding diffusion coefficients. $\boldsymbol{F}_{i}$ and $\boldsymbol{\tau}_{i}$ describe forces and torques acting on particle $i$. The forces $\boldsymbol{F}_{i}$ acting on particle $i$ are given by 


\section{Methods}

the sum of the particle-particle interactions. In addition, we consider interactions with the confining walls of the system in section 4.2 ,

$$
\boldsymbol{F}_{i}=\sum_{i \neq j}\left(\boldsymbol{F}_{i j}^{\mathrm{WCA}}+\boldsymbol{F}_{i j}^{\mathrm{dd}}\right)+\boldsymbol{F}_{i}^{\mathrm{wall}}
$$

The pairwise forces between two particles $i, j$ separated by the distance $r_{i j}=$ $\left|\boldsymbol{r}_{i j}\right|=\left|\boldsymbol{r}_{i}-\boldsymbol{r}_{j}\right|$ consist of forces from excluded volume interactions and dipolar forces.

The forces resulting from excluded volume interactions are modeled by the Weeks-Chandler-Andersen (WCA) pair potential with the interaction energy $\epsilon$ and the particle diameter $\sigma$

$$
\begin{aligned}
\boldsymbol{F}_{i j}^{\mathrm{WCA}} & =-\nabla U^{\mathrm{WCA}}\left(r_{i j}\right) \\
& = \begin{cases}48 \epsilon \frac{r_{i j}}{r_{i j}^{2}}\left[\left(\frac{\sigma}{r_{i j}}\right)^{12}-\frac{1}{2}\left(\frac{\sigma}{r_{i j}}\right)^{6}\right] & \text { if } r_{i j}<\sqrt[6]{2} \sigma \\
0 & \text { if } r_{i j} \geq \sqrt[6]{2} \sigma .\end{cases}
\end{aligned}
$$

The dipolar forces are derived from the pairwise dipole potential [90]

$$
\begin{aligned}
\boldsymbol{F}_{i j}^{\mathrm{dd}}= & -\nabla U^{\mathrm{dd}}\left(\boldsymbol{r}_{i j}, \boldsymbol{\mu}_{i}, \boldsymbol{\mu}_{j}\right) \\
= & \frac{3 \mu_{0} \mu^{2}}{4 \pi r_{i j}^{4}}\left[\hat{\boldsymbol{r}}_{i j}\left(\hat{\boldsymbol{e}}_{i} \cdot \hat{\boldsymbol{e}}_{j}\right)+\hat{\boldsymbol{e}}_{i}\left(\hat{\boldsymbol{r}}_{i j} \cdot \hat{\boldsymbol{e}}_{j}\right)+\hat{\boldsymbol{e}}_{j}\left(\hat{\boldsymbol{r}}_{i j} \cdot \hat{\boldsymbol{e}}_{i}\right)\right. \\
& \left.-5 \hat{\boldsymbol{r}}_{i j}\left(\hat{\boldsymbol{r}}_{i j} \cdot \hat{\boldsymbol{e}}_{i}\right)\left(\hat{\boldsymbol{r}}_{i j} \cdot \hat{\boldsymbol{e}}_{j}\right)\right] .
\end{aligned}
$$

Forces resulting from interactions with the confinement $\boldsymbol{F}_{i}^{\text {wall }}$ are described by the same WCA potential. Fig. 2.3 illustrates the interaction of a particle with the confinement. Here, the shortest distance $\boldsymbol{r}$ to the wall (gray) is measured 


\subsection{Equations of motion}

and the repulsive force for a virtual wall particle (balck dotted circle) with its surface at the point of shortest distance (i.e. outside the wall at a distance $\sigma / 2$ ) is applied. The torques $\boldsymbol{\tau}_{i}$ acting on the particle $i$ result from the pairwise dipolar

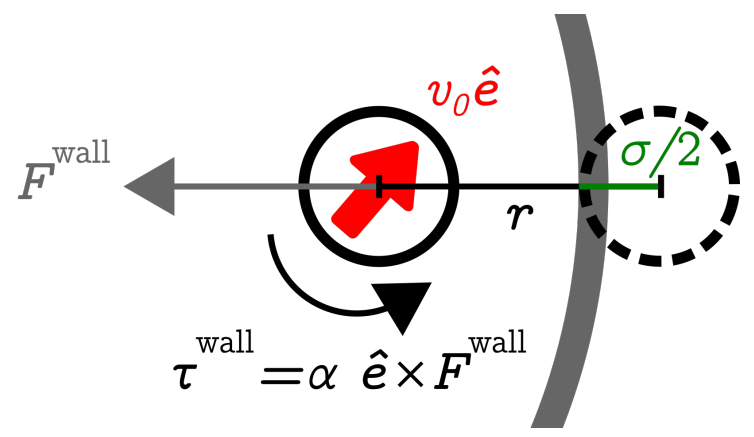

Figure 2.3.: Schematic illustrating particle wall interactions. Repulsive force ( $F^{\text {wall }}$, gray) results from interaction with virtual wall particle (black dotted lines) placed at point of shortest distance outside the wall. Possible torques resulting from wall interaction are shown too $\left(\tau^{\text {wall }}\right.$, black $)$. Orientation of particle is indicated by red arrow.

interactions, interactions with the confining walls of the system and alignment with a homogeneous external magnetic field

$$
\boldsymbol{\tau}_{i}=\sum_{i \neq j} \boldsymbol{\tau}_{i j}^{\mathrm{dd}}+\boldsymbol{\tau}_{i}^{\mathrm{wall}}+\boldsymbol{\tau}_{i}^{\mathrm{B}}
$$

The torques resulting from pairwise dipolar interactions are given by 91

$$
\boldsymbol{\tau}_{i j}^{\mathrm{dd}}=\boldsymbol{r}_{i j} \times \boldsymbol{F}_{i j}^{\mathrm{dd}}=\frac{\mu_{0} \mu^{2}}{4 \pi r_{i j}^{3}}\left[3\left(\hat{\boldsymbol{e}}_{i} \cdot \hat{\boldsymbol{r}}_{i j}\right)\left(\hat{\boldsymbol{e}}_{j} \times \hat{\boldsymbol{r}}_{i j}\right)+\left(\hat{\boldsymbol{e}}_{i} \times \hat{\boldsymbol{e}}_{j}\right)\right]
$$

Active particles may behave differently when approaching a solid boundary. A combination of hydrodynamic effects, steric interactions between the wall and the swimmers' body or, possibly, their flagella can lead to a reorientation of the swimmer at the wall $87,92,94]$. We study such wall induced reorientation of a 


\section{Methods}

particle in section 4.2. We model the reorientation of the particles near a wall by introducing a wall torque $\boldsymbol{\tau}_{i}^{\text {wall }}$ that can be modified with the tuning parameter $\alpha$

$$
\boldsymbol{\tau}_{i}^{\text {wall }}=\alpha \hat{\boldsymbol{e}}_{i} \times \boldsymbol{F}_{i}^{\text {wall }}
$$

A value of $\alpha=0$ results in a purely repulsive wall with no reorienting torque.

In the presence of a homogeneous external field dipolar particles experience no force but an aligning torque

$$
\boldsymbol{\tau}_{i}^{\mathrm{B}}=\mu \hat{\boldsymbol{e}}_{i} \times \boldsymbol{B}(t)
$$

The presence of an external magnetic field can influence the collective behavior of dipolar particles. In chapter 6 we investigate the effect of a constant external magnetic field $\boldsymbol{B}(t)=B \hat{\boldsymbol{e}}_{x}$. Throughout this thesis, the orientation of the external magnetic field is fixed in x-direction $\hat{\boldsymbol{e}}_{x}$.

In chapter 7 , this field will be explicitly time dependent. Here, we study hysteresis in systems of active dipolar particles and how the collective dynamics, in particular structure formation and chain aggregation and separation, are affected. To introduce a constant ramping rate $\beta$ of the magnetic field, we model the time dependency of the external magnetic field as a triangular wave function with amplitude $B$ and period $p=4 / \beta$

$$
\boldsymbol{B}(t)=\left\{B \beta\left|\left[\left(t-\frac{1}{\beta}\right) \bmod \frac{4}{\beta}\right]-\frac{2}{\beta}\right|-B\right\} \hat{\boldsymbol{e}}_{x} .
$$

Fig. 2.4 demonstrates the magnetization protocol used in this thesis. Here, a full magnetization cycle is completed after $5 / 4$ periods. 


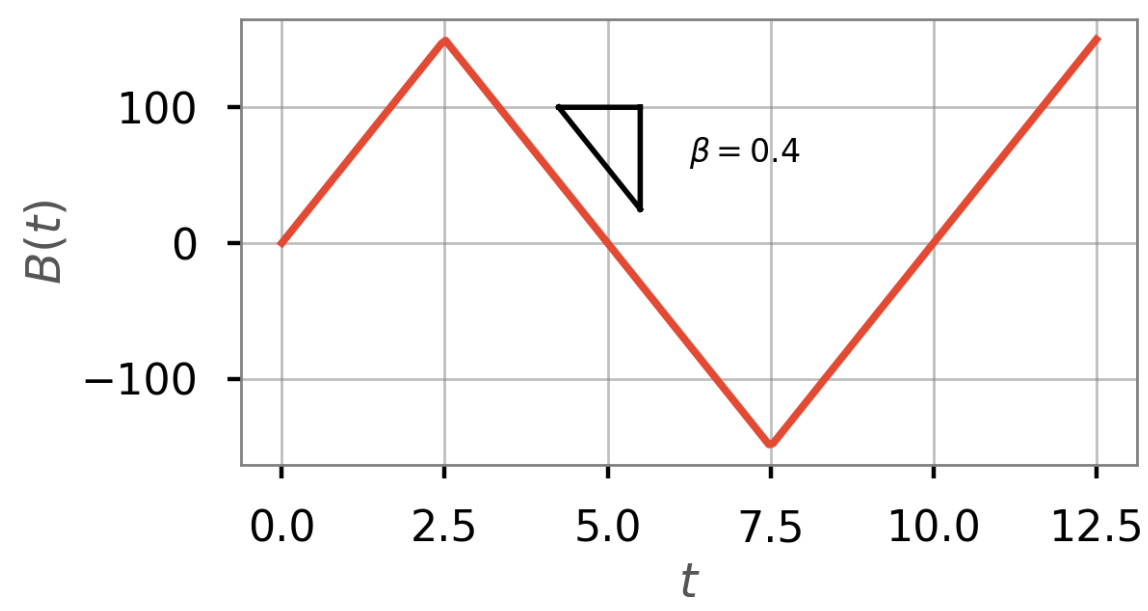

Figure 2.4.: Time dependent external magnetic field as triangular wave function over $5 / 4$ periods with amplitude set to $B=150$ and ramping rate $\beta=0.4$.

\subsection{Identifying clusters and structures of polymers}

It has been shown that particles in (active) dipolar systems self-organize into structured polymers $[40,73,77]$. As a first step to classify structures, we need to identify cluster formation in our system. To identify clusters, we introduced the following criteria for cluster formation. Two particles are considered as bonded, when their distance is smaller than a cut-off distance $r_{i j}<\epsilon$. At least three mutually bonded particles are needed to form a cluster. A density based algorithm (DBSCAN [95]) was used to determine which particles form one cluster. The cutoff distance was chosen based on the nearest neighbor distance $g_{1}(r)$ between pairs of particles in a reference system. As reference system, we chose a system with the magnetic strength $\mu=1$. Here, the dipolar coupling energy of the particles matches the thermal energy $\mu^{2} / \sigma^{3}=T=1$. Since the value of $\epsilon$ depends on the density of the system, we repeated this calculation for the different investigated densities in this thesis. 


\section{Methods}

The relative number of particles that assemble into clusters gives the probability of cluster formation $p_{s}$ 96 98. We calculated the probability of cluster formation varying the self-propulsion speed and magnetic strength of the particles for various systems.

a.

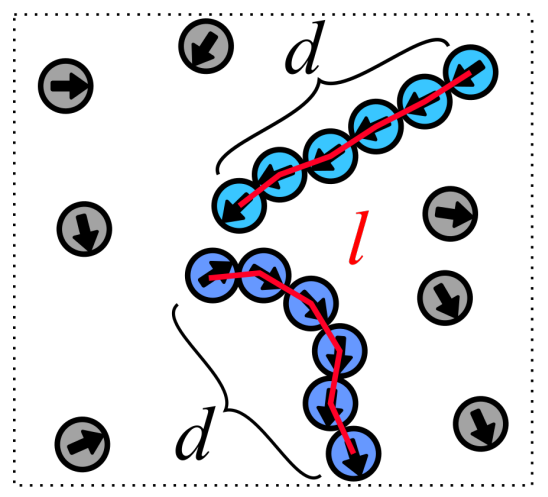

b.

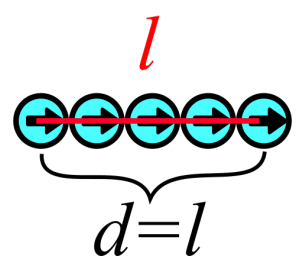

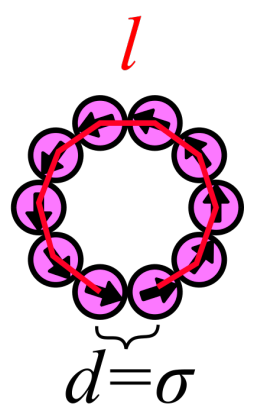

Figure 2.5.: a. Illustration showing how the order parameter $C$ was calculated. Particles in different shades of blue belong to different clusters, gray particles are not in a cluster. The red line indicates the contour length $l$, while $d$ is the headtail distance. The particle orientation is indicated by arrows. b. Example of two extreme configurations: A completely straight chain (cyan), where the head-tail distance $d$ is equal to the contour length $l$ and a perfect ring (magenta), where the head-tail distance $d$ is equal to the particle diameter $\sigma$.

Dipolar particles are expected to self-assemble into chains and rings 41, 42. To distinguish between clusters in ring and chain configuration, equation (2.6) introduces a continuous order parameter ranging between 0 and 1 [99. Figure 2.5 illustrates how this order parameter was calculated.

$$
\mathcal{C}=1-\frac{d-\sigma}{l-\sigma}
$$

Here, $d$ is the head-tail distance while $l$ is the contour length of the identified cluster (color coded). The contour length $l$ was determined by using a shortest- 
path algorithm $[100]$. A completely straight chain $(l=d)$ results in $\mathcal{C}=0$, while a closed ring $(d=\sigma)$ results in $\mathcal{C}=1$.

We then calculated this order parameter for each identified cluster in the system throughout the simulation, resulting in a distribution of the order parameter. The modal value of this distribution is the structure occurring the most for a given parameter combination. Hence, we take this structure as the most representative one. These representative structures were then organized in a diagram of states, depending on the magnetic strength $\mu$ and the self-propulsion speed $v_{0}$ of the particles if the probability of cluster formation $p_{s}>0.5$. Otherwise, the system is assumed to be predominantly unstructured.

\subsubsection{Dynamics of chains and rings}

Because of the geometrical configuration of dipolar particles in ring and chain structures, we expect that rings are rather stationary (no net propulsion), while chains remain motile. To analyze the dynamics of clusters systematically, we tracked the trajectories of individual clusters in chain $(C<0.2)$ or ring configuration $(C>0.8)$ and calculated the mean-squared displacement (MSD) of the center of geometry $(\mathrm{cog})$ of these clusters. To extract the type of motion, we fitted the function $f(t)=4 D_{\operatorname{cog}} t^{\nu}$ to these MSD curves 101, 102, using a least-squares method 103. The MSD curves were obtained using a fast Fourier transform based implementation of a moving window algorithm 104. To reduce the noise contributed by short-lived clusters on the MSD calculation, we analyzed configurations with a life time greater than $t_{\min }=3.6$. 


\section{Methods}

\subsection{Global order parameters}

In large systems, especially at high densities, more complex structures such as branches, connected networks, bands or loops within rings and large scale cluster formation are expected to emerge [38, 73, 77, 78, 105]. Some of these complex structures cannot be properly characterized by equation $(2.6)$, since the head tail distance might not be well defined. To characterize these systems, we used the following three global order parameters introduced in $[73$ instead. To capture small fluctuations in the system, we averaged the global order parameters over the simulation trajectories from $t=18$ to $t=20$ (data taken every $\Delta t=0.1$ ). Similar to other active systems, we expect active dipolar particles to form large clusters. To describe the clustering behavior, we performed a density based cluster analysis, as introduced previously in small systems of active dipolar particles. We determined the value of the cut-off distance $\epsilon$ based on the nearest neighbor distance $g_{1}(r)$ for the three studied densities $(\Phi=0.13,0.23$ and 0.57$)$. To quantify cluster formation, we used the number of particles in the largest cluster $n_{\mathrm{c}}^{*}$ relative to the system size $N$

$$
\phi_{\mathrm{c}}=\frac{n_{\mathrm{c}}^{*}}{N}
$$

The fraction of the largest cluster is close to 0 when particles form small or no clusters and reaches 1 when the whole system forms one giant cluster.

Large systems of active particles are expected to undergo motility induced phase separation (MIPS). In systems of active dipolar particles it has recently been shown that dipolar interactions suppress MIPS [73]. MIPS is expected to occur in systems of active particles without attractive interactions in two dimensions when the average density exceeds the critical density $\Phi_{\text {MIPS }}=0.28[74$. The exact value of 


\subsection{Global order parameters}

the critical density for MIPS has been reported to depend on multiple factors like system size [75] or softness of the particle-particle interaction potential [76].

To correctly identify MIPS, we analyzed the distribution of local densities in the system. A homogeneous system is expected to have a Gaussian distribution of local densities centered around the average system density $\Phi$. A system that undergoes phase separation shows two maxima at the onset of MIPS, one well below the average density one well above. We estimated the local densities by calculating the volumes of the local Voronoi cells $V_{i}$ using Voronoi tessellation, while accounting for periodic boundary conditions [106, 107]. The local density is then given by $\rho_{i}=V_{s} / V_{i}$ from the single particle volume $V_{s}=\pi \sigma^{2} / 4$. To account for the fact that larger volumes naturally occur less frequently, we weighted the probability of the local density $p\left(\rho_{i}\right)$ with its inverse local density.

Because of the aligning torques between dipolar particles, we expect orientational order to emerge, even without the presence of an external magnetic field. We describe the orientational order by calculating the global polarization which is given by the average over the orientations of all particles in the system

$$
\phi_{\mathrm{e}}=\frac{1}{N}\left|\sum_{i} \hat{\boldsymbol{e}}_{i}\right| .
$$

To assess the degree of chain formation in large systems, we applied the following chain criteria, as introduced in 73. Two particles are considered to be bonded if they fulfill the following three criteria simultaneously. Fist, if their distance is smaller than the cut-off distance $r_{i j}<\epsilon$. Second, if they are aligned in parallel $\hat{\boldsymbol{e}}_{i} \cdot \hat{\boldsymbol{e}}_{j}>0$, and third if their orientation is in line with their connecting distance vector $\left(\hat{\boldsymbol{e}}_{i} \times \hat{\boldsymbol{r}}_{i j}\right) \cdot\left(\hat{\boldsymbol{e}}_{j} \times \hat{\boldsymbol{r}}_{i j}\right)>0$. The fraction of particles that fulfill all three 


\section{Methods}

criteria give the degree of polymerization

$$
\phi_{\mathrm{p}}=\frac{n_{\text {chain }}}{N}
$$

We then used combinations of these three order parameters to characterize the state of the system. If an order parameter exceeds a set limit, the system is taken to be in the state corresponding to that order parameter. We chose this limit to be at 0.5 for all three order parameters. For example if $\left\langle\phi_{p}\right\rangle>0.5$ the system is classified as polymerized. Following this classification method the following eight combinations are possible, each characterizing the systems' state as shown in Table 2.1. cluster, oriented gas, gas of chains, oriented cluster, network of chains, oriented chains, bands, disordered gas. We present the results of this classification in diagrams of states in chapter 5 and chapter 6 .

\begin{tabular}{cccl}
$\left\langle\phi_{c}\right\rangle>0.5$ & $\left\langle\phi_{e}\right\rangle>0.5$ & $\left\langle\phi_{p}\right\rangle>0.5$ & state \\
\hline$\checkmark$ & $x$ & $x$ & cluster \\
$x$ & $\checkmark$ & $x$ & oriented gas \\
$x$ & $x$ & $\checkmark$ & gas of chains \\
$\checkmark$ & $\checkmark$ & $x$ & oriented cluster \\
$\checkmark$ & $x$ & $\checkmark$ & network of chains \\
$x$ & $\checkmark$ & $\checkmark$ & oriented chains \\
$\checkmark$ & $\checkmark$ & $\checkmark$ & bands \\
$x$ & $x$ & $x$ & disordered gas \\
\hline \hline
\end{tabular}

Table 2.1.: Order parameter combinations and their corresponding states. 


\section{Computational details}

In this chapter we introduce the simulation methods we used throughout this thesis. We will present the discretized equations of motion and our choice of reduced units. In addition, we give an overview about the simulation parameters we used in our systems.

\subsection{Simulation method}

The in chapter 2 introduced Langevin equations are numerically integrated in two dimensions. In two dimensions, we can simplify equation (2.4) by describing the orientation of the particle with an angle $\varphi$ in the $2 \mathrm{~d}$ plane. The orientation vector in two dimensions is then given by $\hat{\boldsymbol{e}}_{i}=\left(\cos \varphi_{i}, \sin \varphi_{i}\right)$ and the position vector is given by $\boldsymbol{r}_{\boldsymbol{i}}=\left(x_{i}, y_{i}\right)$. The two dimensional equations of motion are then

$$
\begin{aligned}
\dot{\boldsymbol{r}}_{i} & =v_{0} \hat{\boldsymbol{e}}_{i}+\frac{1}{\gamma_{\mathrm{T}}} \boldsymbol{F}_{i}+\sqrt{2 D_{\mathrm{T}}} \boldsymbol{\xi}_{i}^{\mathrm{T}} \\
\dot{\varphi}_{i} & =\frac{1}{\gamma_{\mathrm{R}}} \tau_{i}+\sqrt{2 D_{\mathrm{R}}} \xi_{i}^{\mathrm{R}} .
\end{aligned}
$$

Thus instead of equations $(2.3)$ and (2.4), we integrate equations 3.1 and (3.2). We note that by this change the multiplicative noise in equation (2.4) simplifies 


\section{Computational details}

to additive noise in equation 3.2 .

To integrate the two dimensional equations of motions numerically, we used the following reduced units: time $t^{*}=t D_{0}^{T} / \sigma^{2}$, where $D_{0}^{T}=k_{\mathrm{B}} T / 3 \pi \eta \sigma$ is the translational diffusion constant; position $\boldsymbol{r}^{*}=\boldsymbol{r} / \sigma$; temperature $T^{*}=k_{\mathrm{B}} T / \epsilon$; field strength $B^{*}=B / B_{0}$, where $B_{0}=1 \times 10^{-5} \mathrm{~T}$ is the order of magnitude of the magnetic field strength of the earth; dipole strength $\mu^{*}=\mu B_{0} / \epsilon$.

We note that, with this choice of dimensionless units, the self-propulsion velocity $v_{0}^{*}=v_{0} \sigma / D_{0}^{T}$ is identical to the Peclét number. For the sake of readability, we will omit the asterisk for dimensionless quantities from now on.

Systems that contain and model interactions with confining walls are simulated using overdamped Brownian dynamics (BD) simulations where the dimensionless equations of motions are integrated using a 2nd order Runge-Kutta scheme 108 , 109] (see equations (3.5) and (3.6)). Here, forces and torques are calculated in two steps, an estimator (superscript e) and a corrector (superscript c) step. During the estimator step, forces and torques are being calculated based on the positions and orientations of the particles at time $t$, while the corrector step is based on forward-in-time positions $\tilde{\boldsymbol{r}}_{i}$ and orientational angles $\tilde{\varphi}_{i}$ :

$$
\begin{aligned}
& \tilde{\boldsymbol{r}}_{i}=\boldsymbol{r}_{i}(t)+v_{0} \hat{\boldsymbol{e}}_{i} \Delta t+\frac{1}{T} \boldsymbol{F}_{i}^{\mathrm{e}}\left(\boldsymbol{r}_{i}, \varphi_{i}\right) \Delta t+\sqrt{2 \Delta t} \boldsymbol{\xi}_{i}^{\mathrm{T}}(t) \\
& \tilde{\varphi}_{i}=\varphi_{i}(t)+\frac{3}{T} \tau_{i}^{\mathrm{e}}\left(\boldsymbol{r}_{i}, \varphi_{i}\right) \Delta t+\sqrt{6 \Delta t} \xi_{i}^{\mathrm{R}}(t) .
\end{aligned}
$$

The Runge-Kutta integration step then takes the average of the estimator and corrector forces and torques to calculate the positions and orientational angles at 


\subsection{Simulation method}

time $t+\Delta t$ :

$$
\begin{aligned}
\boldsymbol{r}_{i}(t+\Delta t)= & \boldsymbol{r}_{i}(t)+v_{0} \hat{\boldsymbol{e}}_{i} \Delta t \\
& +\frac{1}{2 T}\left[\boldsymbol{F}_{i}^{\mathrm{e}}\left(\boldsymbol{r}_{i}, \varphi_{i}\right)+\boldsymbol{F}_{i}^{\mathrm{c}}\left(\tilde{\boldsymbol{r}}_{i}, \tilde{\varphi}_{i}\right)\right] \Delta t \\
& +\sqrt{2 \Delta t} \boldsymbol{\xi}_{i}^{\mathrm{T}}(t) \\
\varphi_{i}(t+\Delta t)= & \varphi_{i}(t) \\
& +\frac{3}{2 T}\left[\tau_{i}^{\mathrm{e}}\left(\boldsymbol{r}_{i}, \varphi_{i}\right)+\tau_{i}^{\mathrm{c}}\left(\tilde{\boldsymbol{r}}_{i}, \tilde{\varphi}_{i}\right)\right] \Delta t \\
& +\sqrt{6 \Delta t} \xi_{i}^{\mathrm{R}}(t) .
\end{aligned}
$$

Gaussian distributed random numbers $\boldsymbol{\xi}_{i}^{\alpha}(t)$ were generated by the Mersenne Twister random number generator [110].

Simulations with periodic boundary conditions, in particular large systems with $N=1156$ particles with and without (time dependent) external magnetic field, were realized by using the simulation toolkit HOOMD-blue vers. 2.6 [111, 112]. The active self-propulsion of the particles and alignment in an external (timedependent) magnetic field were added by in-house developed modules. To avoid interactions between periodic images of the particles, a cut-off distance $r_{\text {cut }}=$ $L / 2-\sigma$ was introduced for dipolar interactions. The project data and its parameter space was managed within a signac framework 113, 114. Parameters in HOOMD were chosen to match the parameters of the Runge-Kutta scheme simulations. In particular, constants for the translational and rotational diffusion of the spherical particles match as well as the magnetic moments and self-propulsion speeds.

We categorize our simulations into four parts: small systems, large systems without an external field, large systems with a constant external field, and large 


\section{Computational details}

systems with a time dependent external field. We now introduce the choices for the main simulation parameters for the four parts. Detailed information about the simulations are listed in appendix A. Throughout all simulations the temperature of the system was set to $T=1$, while varying the self-propulsion speed $v_{0}$ and the magnetic moment $\mu$ of the particles. Particles were initially placed on a regular quadratic grid, while the grid spacing was adjusted depending on density. The following paragraphs introduce the additional system specific parameter.

\section{Simulation parameter small systems}

We analyzed small systems with $N=36$ particles for various boundary conditions and sizes of the simulation box. First, we performed simulations of small systems with periodic boundary conditions where the side length was set to $L=50(\Phi=$ 0.01). In a next step, confining walls were added to this system. To investigate the effect of the geometry of the confinement, squared confining walls and circular confining walls were chosen. To match the area of the squared confinement, the radius of the circular confinement was set to $R=L / \sqrt{\pi}$. Here, we varied the geometry of the confinement and the wall torque parameter $\alpha=0.0,0.4$ and 2.0.

In a next step, we investigated how density affects cluster formation and modulated the density by increasing the particles number $N=81$, as well as by reducing the size of the simulation box in squared confinement with $\alpha=0$. We studied three densities resulting from the following particle number and box size combinations: $\Phi=0.01(N=36, L=50), \Phi=0.03(N=81, L=50), \Phi=0.03(N=36$, $L=33.3)$ and $\Phi=0.1(N=36, L=16.8)$

For all parameter combinations, numerical integration was performed for $N_{\mathrm{t}}=$ $1 \times 10^{6}$ time steps with a time step width $\Delta t=2 \times 10^{-5}$ in small systems of active 


\subsection{Simulation method}

dipolar particles.

\section{Simulation parameter large systems without external field}

To investigate the collective dynamics and structure formation of dipolar active particles, simulations in large systems with $N=1156$ particles in periodic simulation boxes were performed with HOOMD-blue with an in-house developed module for the activity of the particles. To study the effect of density, we varied the side length of the simulation box. We chose the following three densities. One well below $(\Phi=0.13, L=85)$, one close to $(\Phi=0.23, L=63)$ and one above $(\Phi=0.57, L=40)$ the critical density were MIPS is expected in systems of active particles.

\section{Simulation parameter large systems with constant external field}

To investigate the effect of a constant homogeneous external field on the structures and on the collective dynamics of dipolar active particles, we varied the strength $B$ of the external field. Alignment of individual particles with the magnetic field depends on the magnetic moment of the particle itself. Therefore, different combinations of the magnetic moment and field strength $B$ were explored. We studied the effect of activity in these systems by varying the self-propulsion speed $v_{0}$. Simulations were performed with HOOMD-blue with an in-house modification of the external magnetic field.

\section{Simulation parameter large systems with time dependent external field}

Time dependent external magnetic fields are used to control magnetic swimmers externally. This method is especially promising in biomedical applications i.e. drug 


\section{Computational details}

delivery. The effect of an external magnetic field on a single particle is well studied. Here, we are interested in how an external time dependent magnetic field affects the collective behavior of active dipolar particles in bulk suspension. Ferromagnetic materials are expected to show hysteresis in a time dependent magnetic field. Analyzing such hysteresis curves gives access to important magnetic properties of the material. Here, we investigate how activity of dipolar particles affects hysteresis curves. The external time dependent magnetic field was modeled using a triangular wave with period $p=4 / \beta$ and amplitude $B$ and implemented in an in-house developed module of HOOMD-blue. Simulations were run over 5/4 periods, with a time-step width $\Delta t=1 \times 10^{-5}$ for $N_{\mathrm{t}}=\left\lfloor\frac{5}{\beta \Delta t}\right\rceil$ time steps. Simulations were performed for values $\beta=0.4,0.9,1.9$ and 4.0 which results in $N_{\mathrm{t}}=1.25 \times 10^{6}$, $5.56 \times 10^{5}, 2.63 \times 10^{5}$ and $1.25 \times 10^{5}$ time steps respectively. 


\section{Structure formation in small systems}

Because dipolar particles are expected to form chain and ring structures in passive systems, we study how activity affects those structures in this chapter. In addition, we investigate how interactions with confinement influences structure formation of dipolar active particles in small systems. We quantify the type of structures that emerge and demonstrate how the dominant structures are affected by the magnetic moment, self-propulsion speed and confinement.

Partial results presented in this chapter, in particular results about structure formation in confinement have been published in V. Telezki and S. Klumpp, "Simulations of structure formation by confined dipolar active particles", Soft Matter 16, 10537-10547 (2020), In some cases, wording and figures have been adapted to match the format of this thesis. V.T. and S.K. conceived of the presented project. V.T. set up and carried out the numerical simulations and analyzed the data. S.K. supervised the findings of this work. Both authors discussed the results and contributed to the final manuscript. 


\section{Structure formation in small systems}

\subsection{Assembly of chain and ring structures}

In a first step, we investigated the dynamics of a small number of particles $(N=$ 36) without the presence of boundaries (periodic boundary conditions). In the simulations we observed that for certain parameters dipolar active particles selfassemble into clusters. A cut-out of a typical snapshot of the simulation is shown in Figure 4.1. These clusters were mainly found to adopt two distinct structural formations: chains (Figure $4.1 \mathrm{a}-\mathrm{c}$ ) and rings (Figure $4.1 \mathrm{~d}$ ). We observed that

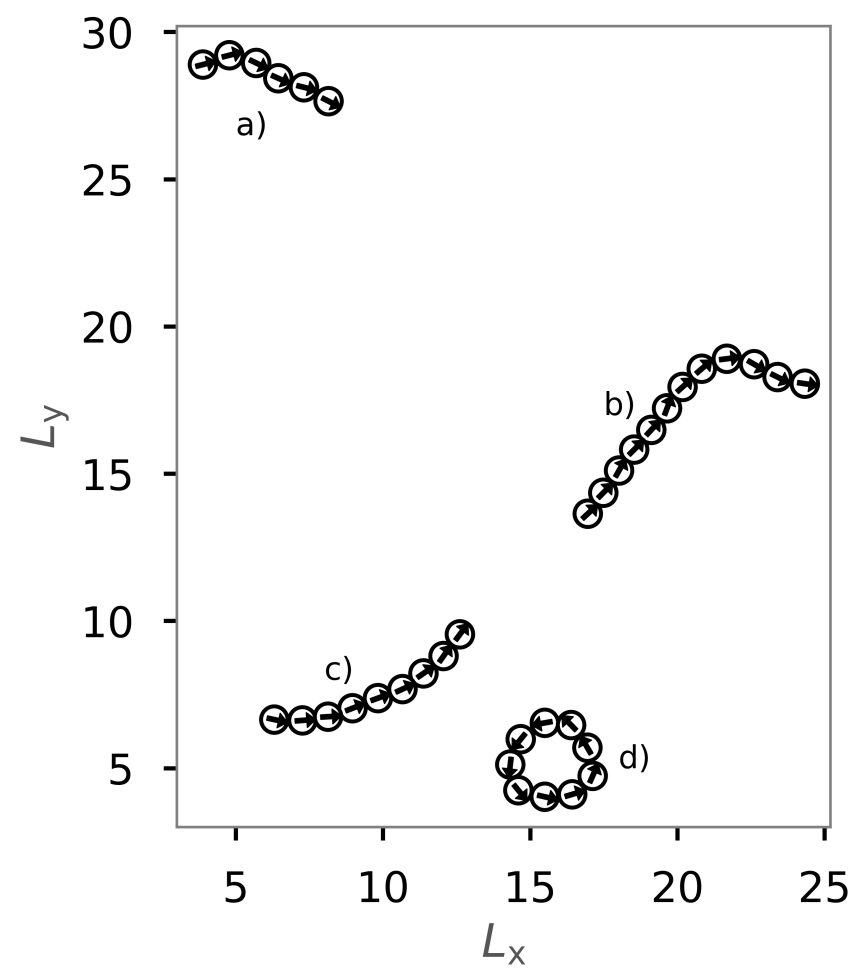

Figure 4.1.: Detail of a snapshot at time $t=45.50$ for a simulation with $v_{0}=$ $10, \mu=1.75$ in a squared simulation box with $L=50$ and periodic boundary conditions with $N=36$ particles. The magnetic moment of the dipolar particle is represented as a black arrow. Different chain conformations (a-c) and a ring conformation (d) are visible.

individual dipolar active particles usually first self-assemble into a chain which then 


\subsection{Assembly of chain and ring structures}

eventually catches its own tail and forms a ring. These structures are dynamic; rings and chains can disassemble again into chains, smaller chains or individual monomers. The number of particles in one structure varies. Generally, we observed that the structure depends on the magnetic moment $\mu$, the self-propulsion speed $v_{0}$ and the boundary conditions.

To quantify the formation of these structured clusters, we asked what the probability for cluster formation $p_{\mathrm{s}}$ is and how it depends on the magnetic strength $\mu$ and the self-propulsion speed $v_{0}$. To answer that question, we identified particles forming a cluster using a density based algorithm (see chapter 2), where the closest neighbor distance was set to $\epsilon=1.21$ and the minimal number of particles in a neighborhood to form a cluster was set to $N=3$ which is similar to other studies 73, 98. The value of $\epsilon$ was determined by analyzing the nearest neighbor distance $g_{1}(r)$, for $\mu=1$ (see Figure 4.2). The histograms indicate that the closest neighbor distance weakly depends on the self-propulsion speed $v_{0}$. Here, $\epsilon$ is highlighted by the dotted line. This value of $\epsilon$ was chosen as more than $85 \%$ of the particles lay in this region and well behind the shoulder of the distributions for all three choices of the self-propulsion velocity.

Based on this value for $\epsilon$, the probability for cluster formation was calculated, averaged over 10 realizations. The result is shown in Figure 4.3 for different selfpropulsion speeds $v_{0}$. Here, we observe a steep increase of the probability of cluster formation $p_{\mathrm{s}}$ with increasing magnetic strength $\mu$. This steep increase suggests that a critical value $\mu^{*}$ for the magnetic strength can be defined. We estimated such critical value by calculating $p_{\mathrm{s}}\left(\mu^{*}\right)=0.5$ via linear interpolation between the two data points closest to $p_{\mathrm{s}}=0.5$. The inset shows that the critical magnetic strength $\mu^{*}$ increases approximately linearly with the self-propulsion speed $v_{0}$. We 

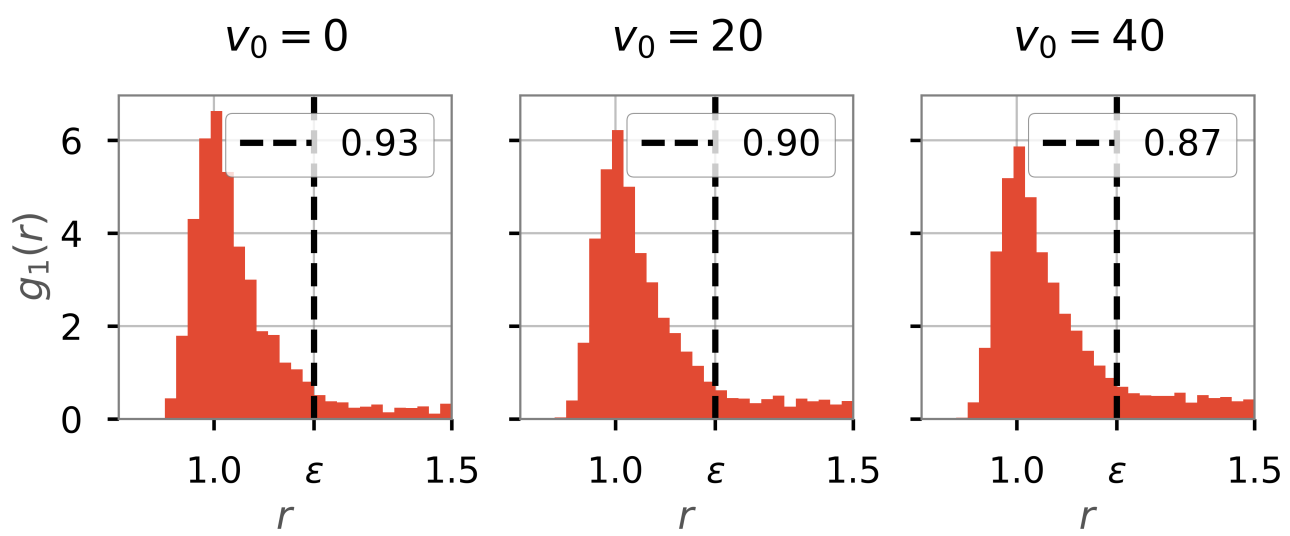

Figure 4.2.: Normalized histograms of the nearest neighbor distance $g_{1}(r)$ for dipolar particles with $\mu=1$ and different self-propulsion speeds $v_{0}$. The dotted line is drawn at $\epsilon=1.21$. The number in the top right shows the fraction of accumulated counts up to the dotted line. Data taken from 10 simulation runs with pbc and $L=50$.

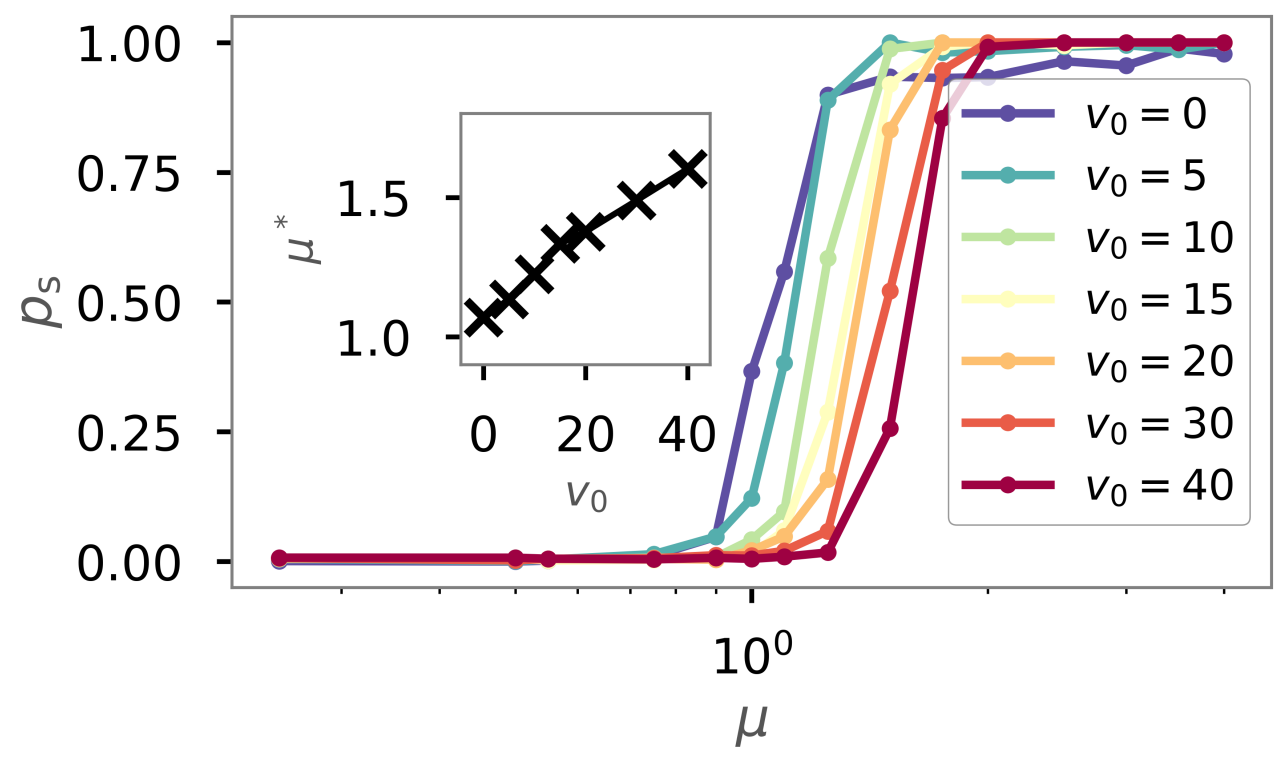

Figure 4.3.: Semi-logarithmic curves of the probability of cluster formation $p_{\mathrm{s}}$ as a function of the magnetic strength $\mu$ for $N=36$ particles. The different curves correspond to different self-propulsion speeds $v_{0}$. The inset shows the dependence of the critical magnetic strength $\mu^{*}$ on the self-propulsion speed. Data taken from 10 simulation runs with $L=50$ (pbc). 
note that the critical value of $\mu^{*} \approx 1.0$ for passive particles $\left(v_{0}=0\right)$ corresponds to a dipolar coupling strength of $\lambda=\mu^{* 2} / T=1$. For passive particles, the ratio between pairwise dipolar coupling energy and thermal energy is the only relevant parameter for cluster formation. Clusters begin to dominate $\left(p_{\mathrm{s}}>0.5\right)$ exactly when dipolar coupling energy between two particles matches the thermal energy of the system $\left(\mu^{* 2}=T\right)$. For systems below the critical density required for MIPS, adding activity to the system increases the drive towards disorder and thus requires stronger dipolar interactions for clusters to form.
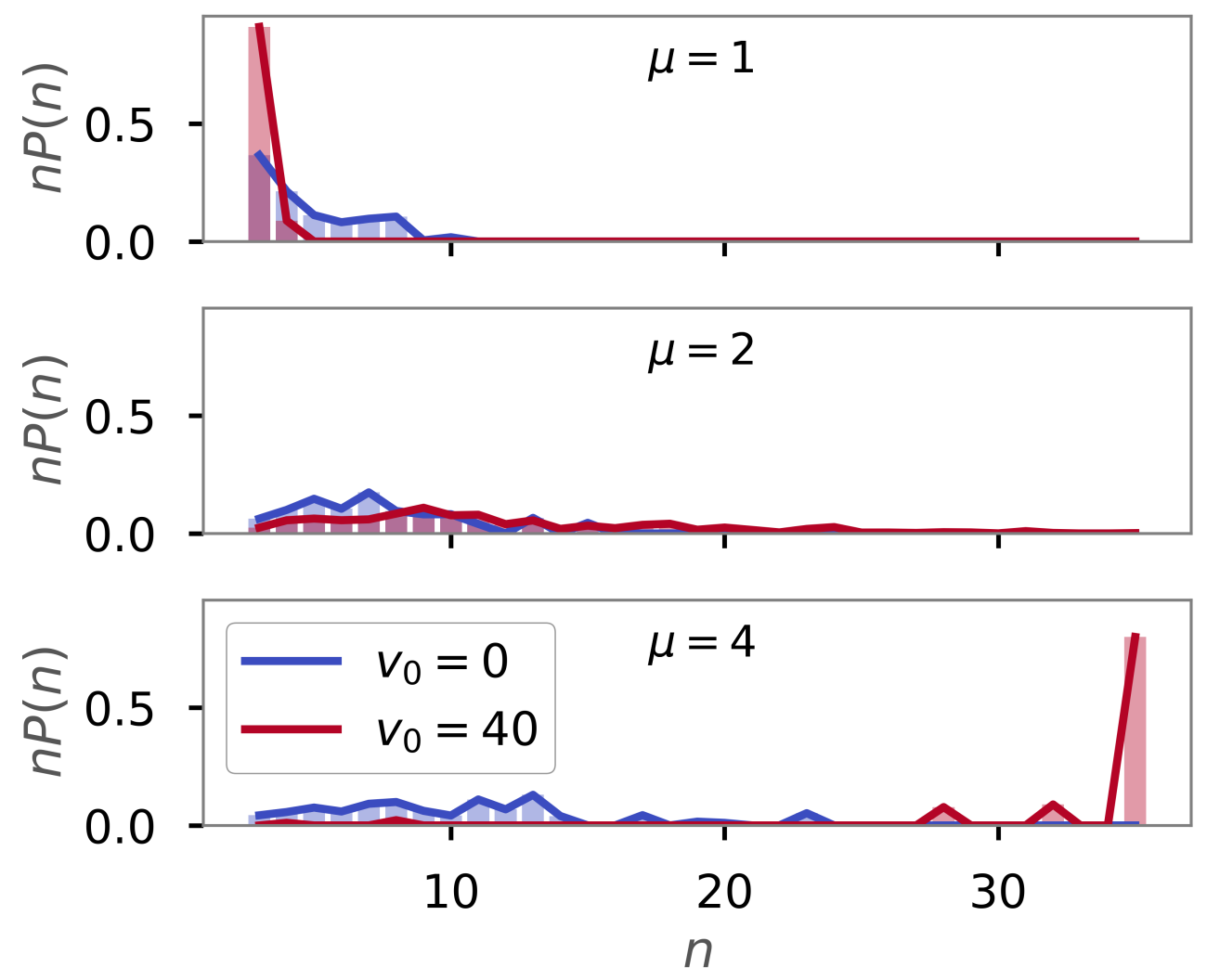

Figure 4.4.: Weighted cluster size distributions $n P(n)$ for passive $\left(v_{0}=0\right.$, blue) and active $\left(v_{0}=40\right.$, red $)$ particles for different magnetic strengths $\mu$. Data taken from 10 simulation runs with $N=36$ and $L=50$ (pbc).

In addition to the probability of cluster formation, we analyzed the weighted 


\section{Structure formation in small systems}

cluster size distribution $n P(n)$ for different magnetic strengths and its dependence on self-propulsion speeds. The results are shown in Figure 4.4. For weak dipolar interactions $(\mu=1)$, small cluster sizes dominate the distribution, independent of the self-propulsion speeds. With increasing dipolar strength, we noticed that passive particles (blue) tend to organize in small clusters ( $n=3$ to 7$)$ while active particles (red) tend to form one cluster consisting of all particles in the system $(n=36)$. This effect seems to be more evident for higher values of $\mu$.

\subsubsection{Classification of structures}

After determining the probability that a cluster forms, we classified the types of structures (i.e chains and rings) that we observed according to the order parameter introduced in equation (2.6). Here, we ask the question what value of the order parameter represents the system the best for given self-propulsion speed and magnetic strength. Do we observe structures and if yes, are they more likely to be chains or rings?

Fig. 4.5 shows examples of the cluster size weighted histograms for different simulation parameters. Each histogram is based on 10 independent realizations. To find the most representative state, we first determined whether more states with structure than without a structure were counted. This characteristic is given by the probability of cluster formation. If the probability of cluster formation $p_{\mathrm{s}}>0.5$, we determined which structure occurred most frequently by calculating the maximum $C_{\max }$ (e.g. colored bar in Figure 4.5 for $\mu=2.0, v_{0}=0$ : cyan, and $\mu=4.0, v_{0}=40:$ magenta). This structure is then assumed to be the best representation of the system for given parameters. If $p_{\mathrm{s}}<0.5$ we did not calculate 
the maximum (e.g. Figure 4.5 for $\mu=1.0, v_{0}=0$ : all bars gray). Here, the system is predominantly in an unstructured state.

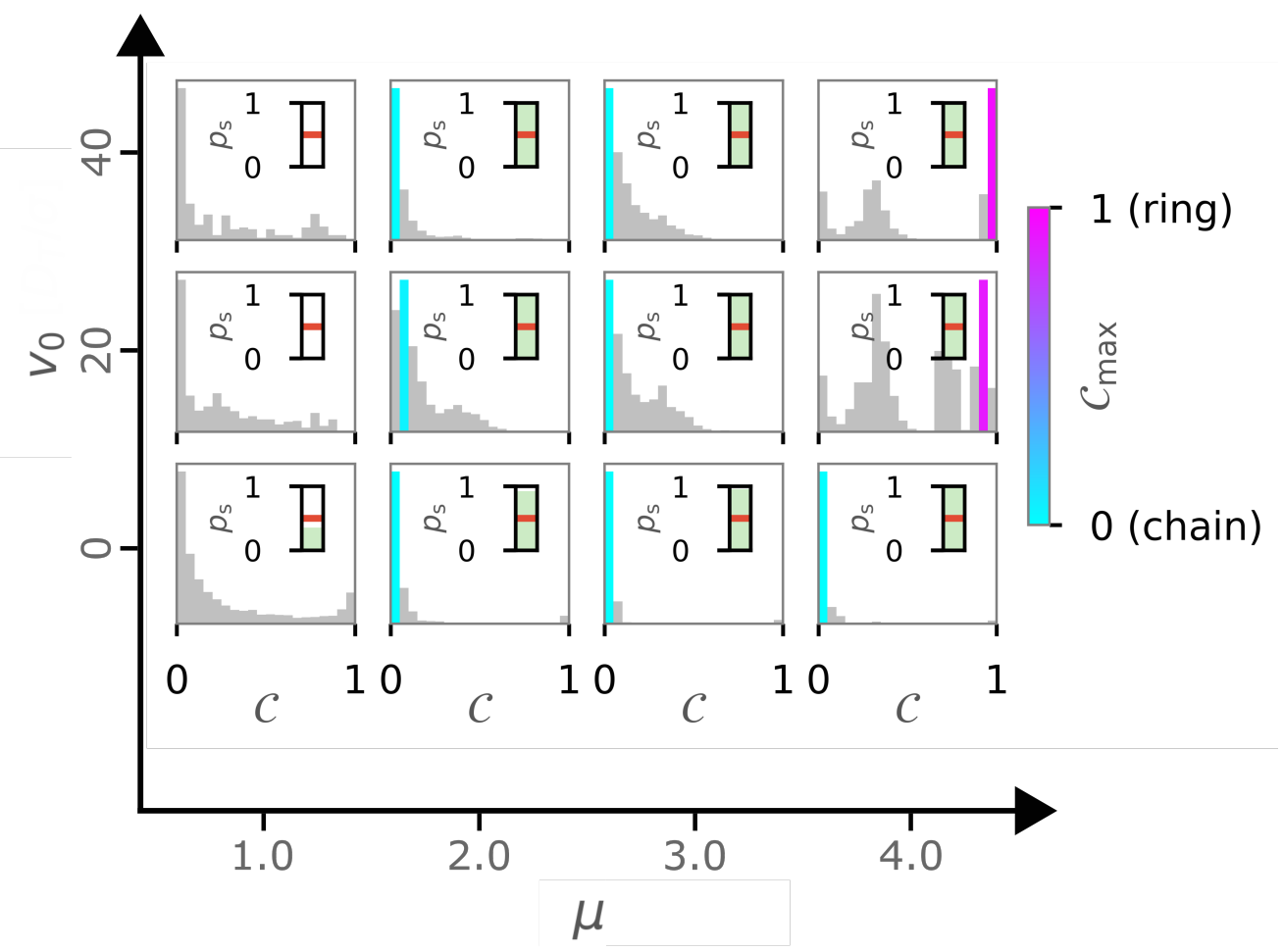

Figure 4.5.: State weighted histograms for $N=36$ particles $L=50$ (pbc) of the order parameter $C$ for different simulation parameters $v_{0}$ and $\mu$. The maximum of the histogram is taken as the most representative structure if the probability of cluster formation $p_{\mathrm{s}}>0.5$ (inset, red line). In these cases the maxima are colored according to the order parameter $C$. Chain-like structures are represented by values of $C \approx 0$ (cyan), while ring like structures are represented by $C \approx 1$ (magenta). If $p_{\mathrm{s}}<0.5$, the system is considered as predominantly unstructured and all bars are shown in gray. Data taken from 10 simulation runs.

We then continued to calculate $C_{\max }$ while varying the self-propulsion speed $v_{0}$ and the magnetic strength $\mu$ and display $C_{\max }$ in a diagram of states (Figure 4.6). The diagram of states shows that the structure depends on both the magnetic strength $\mu$ and the self-propulsion speed $v_{0}$ of the particles. For low magnetic strengths $(\mu \leq 1.0)$ we observed no structure formation, independent of the self- 


\section{Structure formation in small systems}

propulsion speed (as already shown in Figure 4.3). For higher magnetic strengths, we observed chain and ring formation, depending on the self-propulsion speed. The results shown in Figure 4.6 are qualitatively similar to bigger systems of dipolar active particles without confinement [73]. Importantly, by distinguishing the structured state more carefully (i.e. chains and rings) we can show that by changing the self-propulsion speed while keeping the magnetic strength constant one can influence the configuration of the dipolar particles. For example, the structure changes from a chain $\left(v_{0}=0\right)$ to a ring $\left(v_{0}=20\right)$ and back to a chain structure $\left(v_{0}=30\right)$ while keeping the magnetic strength constant at $\mu=3.5$.

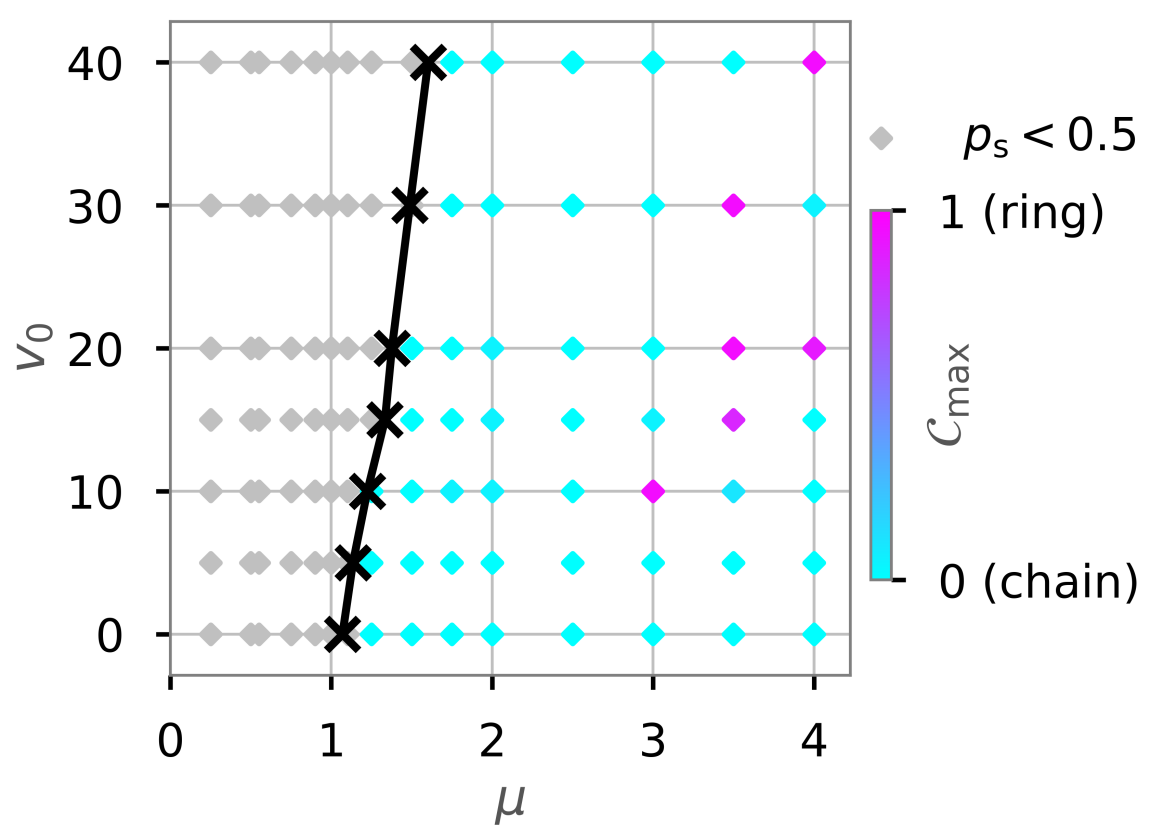

Figure 4.6.: Diagram of states for $N=36$ particles in a system with $L=50$ (pbc) according to the maximum of the order parameter $C_{\max }$. Here, the magnetic strength $\mu$ and the self-propulsion speed $v_{0}$ are varied. The crosses indicate the critical magnetic moment $\mu^{*}$ for structure formation $\left(p_{\mathrm{s}}=0.5\right)$ from Figure 4.3 . The colored areas and the lines were added as visual aids.

For small systems with $N=36$ particles and periodic boundary conditions, chain 


\subsection{Influence of confinement on structure formation}

structures dominate the diagram of states when the magnetic strength exceeds the critical value for cluster to form $\left(\mu>\mu^{*}\left(v_{0}\right)\right)$. In the case of passive particles $\left(v_{0}=0\right)$ this result does not fully match earlier analytical studies where ring structures were predicted to dominate for values $\mu>\mu_{\text {ring }}^{*}[41,52,53]$. In analytical calculations, the ratio of chain to ring structures is independent of the density of the system. In our simulations of finite length we observed that passive particles tend to form smaller clusters than active ones (see Figure 4.4). We argue that in our dilute systems $(\Phi=0.01)$ passive particles interact locally and tend to form sub-systems of 3 to 4 particles that then interact with other sub-systems on much longer time scales, as suggested by the results of the Monte Carlo simulations presented in [53]. For these sub-system sizes, chain structures were predicted to dominate, which is in line with our observations.

\subsection{Influence of confinement on structure formation}

Typically, active particles are confined by different geometries during experiments like microtraps (circular) 115 or channels and chambers in microfluidic devices (squared). To investigate the effect of confinement on structure formation in small systems, we varied both the geometry and the type of interactions between the particle and the confining walls in this section. Specifically, we considered that active particles tend to reorient at walls and added a wall torque, as previously introduced in equation 2.5 .

To analyze how the variation of the boundary conditions affects a single dipolar swimmer, we first simulated a single active particle and computed the probability density. The results of ensemble and time averages over $243 \times 10^{6}$ snapshots are 


\section{Structure formation in small systems}

shown in Figure 4.7 for squared and circular confinement and for three different torque parameters $\alpha=0.0,0.4$ and 2.0 .
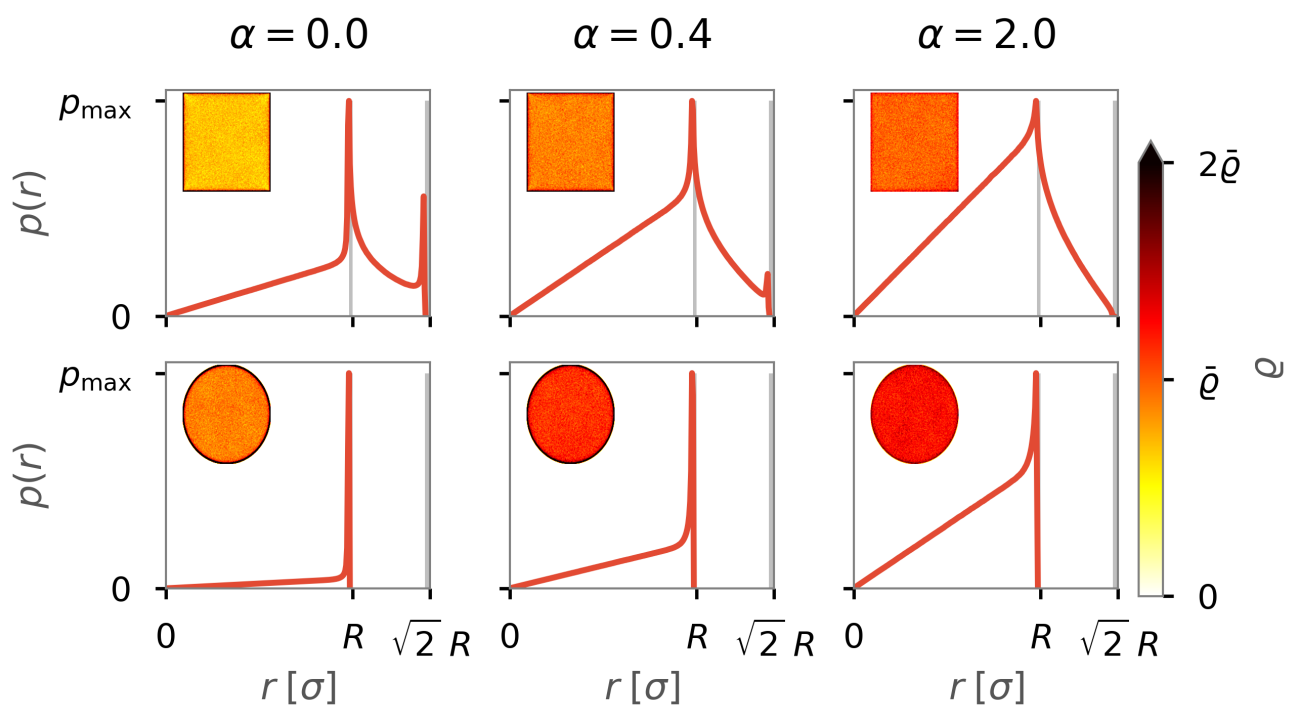

Figure 4.7.: Two-dimensional probability density (heat map in the inset) and radial probability density $p(r)$ for one active particle in squared and circular confinement. The distributions were obtained from $243 \times 10^{6}$ snapshots of a single particle with self-propulsion speed $v_{0}=20$ (averaged over ensemble and time). The geometry (top row: squared, bottom row: circular) and the interactions with the walls were varied, using purely repelling walls $(\alpha=0.0)$ and walls with an additionally induced torque ( $\alpha=0.4$ and 2.0). The side length of the box is $L=50$. The radius of the circular confinement was chosen to match the area of the squared confinement. Volume that cannot be occupied by a particle is marked in gray. The color map is cut off at two times the average probability density $2 \bar{\varrho}$.

We identified two regions; a homogeneous probability density in the interior of the confinement $(r<R)$, where the radial probability density is linear, and a second region directly at the side walls $(r=R)$ or at the corners $(r=\sqrt{2} R)$ of the confinement where the radial probability density drastically increases and shows peaks. With an additional wall induced torque $(\alpha \neq 0)$, the relative probability density is increased in the interior with decreased peaks at the walls $(r=R)$ or in the corners $(r=\sqrt{2} R)$. 


\subsection{Influence of confinement on structure formation}

The relative probability density of one active particle shows that active particles without a reorienting torque tend to get trapped at the boundaries, especially in the corners of a box. Introducing a wall torque results in a more homogeneous probability density, irrespective of the geometry. In the case of squared confinement, a high wall induced torque $(\alpha=2)$ prevents trapping in the corners. Trapping at the walls and in corners is reduced by the wall torque, as particles do not have to rely on thermal fluctuations for their reorientation. A recent combined experimental and numerical study has presented similar probability densities of magnetotactic bacteria (MSR-1) swimming in circular microtraps 115. This study reported curvature dependent values for $\alpha=2-4$ for similar sized circular confinements, which is in agreement with our observations.

Next, we analyzed how structure formation of $N=36$ dipolar active particles is affected by the confining walls. In movies of the simulation, we noticed that the formation of rings is often initiated by collisions with walls. To test the hypothesis that ring formation is initiated by the walls of the confinement, we calculated the local order parameter $C_{\max }(\boldsymbol{r})$ for each position $\boldsymbol{r}$ on a 250 by 250 grid in the confinement over 10 realizations. The results for moderate swimming speed $v_{0}=20$ and magnetic strength $\mu=2$ for different wall interactions and geometries are shown in Figure 4.8 ,

Rings (magenta) form predominantly at the walls for the squared (top row) and the circular (bottom row) confinement, irrespective of the wall torque $\alpha$. In the case of the squared confinement, ring formation also occurs in the corners of the confinement. These results illustrate that ring formation is predominantly caused by interactions with the walls of the confinement and that this mechanism is not strongly affected by the geometry or wall induced torques. 


$$
\alpha=0.0
$$
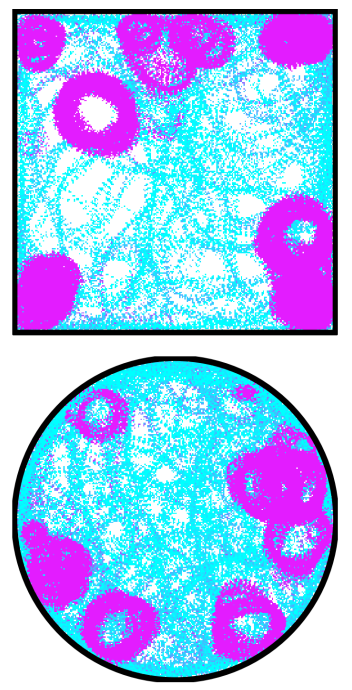

$\alpha=0.4$
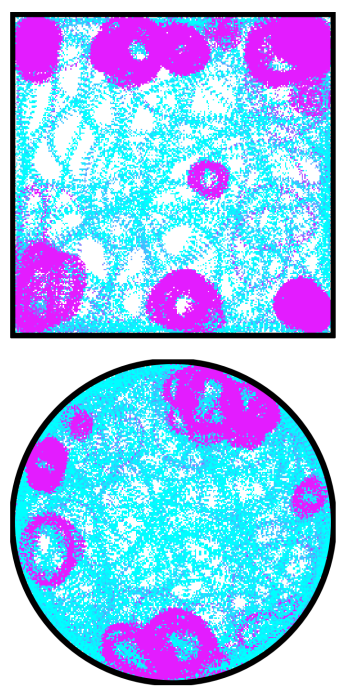

$\alpha=2.0$

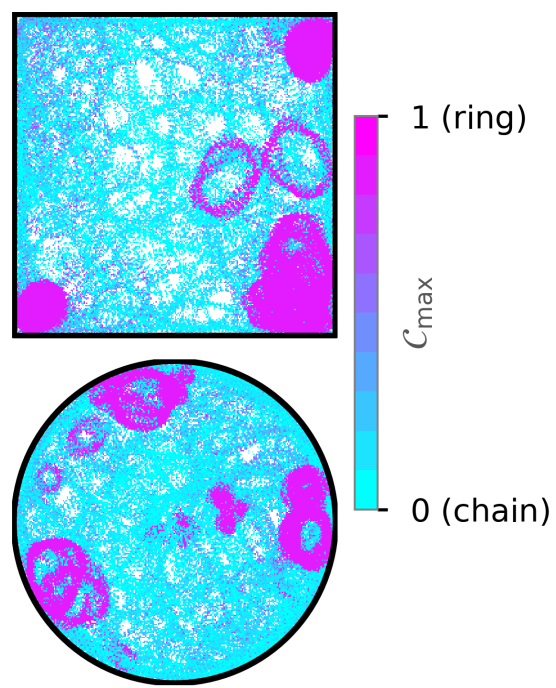

Figure 4.8.: Local order parameter $C_{\max }(\boldsymbol{r})$ for squared confinement (top row) and circular confinement (bottom row) with different induced wall torques $\alpha=$ 0.0, 0.4 and 2.0. The color bar represents the local order parameter. The local order parameter was calculated on a 250 by 250 grid for a self-propulsion speed $v_{0}=15$ and a magnetic strength $\mu=2$ over 10 realizations. The side length of the box is $L=50($ circle $R=50 / \sqrt{\pi})$.

In contrast to systems with confinement, we observed fewer ring configurations in systems with periodic boundary conditions. This deficit in ring configurations in systems with periodic boundary conditions together with the increase in ring formation due to walls suggests that ring formation is driven by the increase of probability density at walls and the accompanied probability fluxes. A recent study has systematically investigated the emergent probability fluxes in confined systems and their impact on trajectories of active particles 85.

In addition, Figure 4.8 indicates that rings are rather stationary, while chains remain motile. As introduced in section 2.2.1, we proceeded to analyze the dynamics of clusters systematically. Fig. 4.9 shows the fitted exponent $\nu$ and the prefactor $D_{\operatorname{cog}}$ against the order parameter $C$ of the tracked clusters. For rings 


\subsection{Influence of confinement on structure formation}

$(C>0.8)$, the exponent is mainly evenly distributed between 0.5 and 1.5 , revealing that rings show diffusive behavior $(\nu=1)$ with some rings being sub- $(\nu<1)$ and superdiffusive $(\nu>1)$. For chains $(C<0.2)$ the exponent is distributed between 1 and 2 (while occurring more frequently at higher values of $\nu$ ) which shows the superdiffusive behavior of chains. We note that truly ballistic motion $(\nu=2)$ is not reached. Since the exponent $\nu$ was determined over all available time scales, the typical diffusive behavior of active particles for short times contributes to reducing the measured exponents to $\nu<2$. The low proportionality constant for rings proves together with the exponent $\nu$ that rings diffusive slowly or can be considered stationary $\left(D_{\operatorname{cog}} \approx 0\right)$ for the analyzed time scales. Chains on the other hand show superdiffusive motion where the factor $D_{\operatorname{cog}}$ increases with the self-propulsion velocity $v_{0}$, as expected.

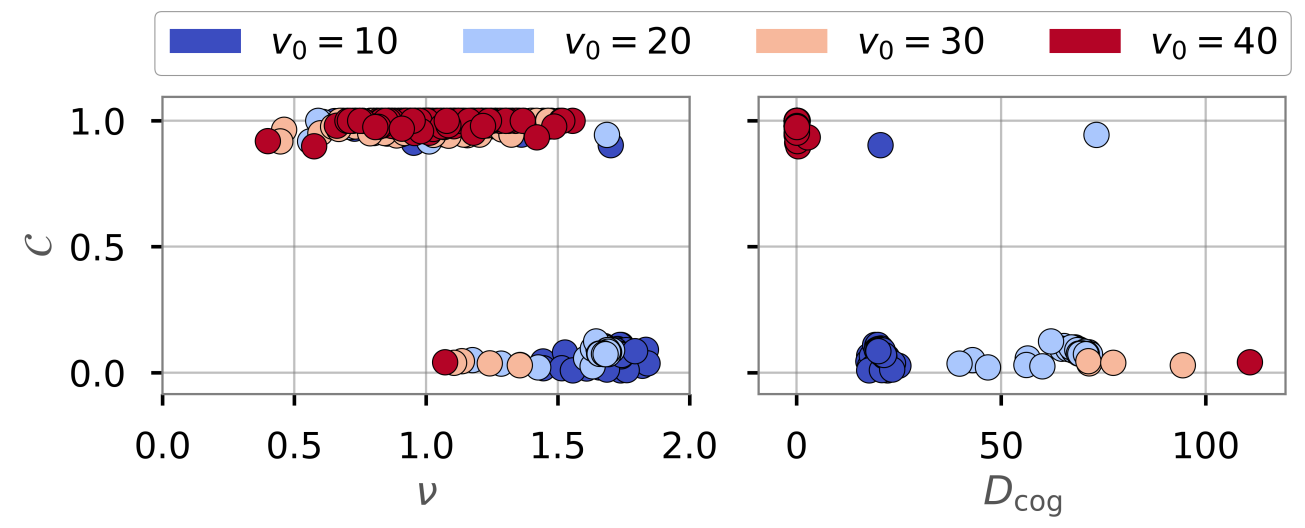

Figure 4.9.: Scatter plots of the exponent $\nu$ and the factor $D_{\operatorname{cog}}$ against the order parameter $C$ obtained from mean-squared displacements of the center of geometry of clusters in either chain $(C<0.2)$ or ring $(C>0.8)$ configuration (we note that clusters with intermediate values of $C$ were not included in this analysis). Data taken from 10 realizations of simulations for the parameters $v_{0}=[10,20,30,40]$ (blue to red), $\mu=[1.5,2.0,2.5,3.0]$ for both circular and quadratic confinement with $L=50$ and $\alpha=[0.0,0.4,2.0]$.

Next, we ask the question how do these different wall geometries and wall prop- 


\section{Structure formation in small systems}

erties affect structures globally? To answer that question we are again interested in the most representative structure formed by the dipolar active particles. We therefore determined diagrams of states based on the maximum of the order parameter $C_{\max }$ while varying the boundary conditions. The results are shown in Figure 4.10. The rows show the dependence on the geometry (top row box, bottom row circle) and the columns the effect of the additional wall torque $(\alpha=0.0,0.4$ and 2.0). The comparison between the diagrams of states shows that boundary conditions affect structure formation to some extent. We noticed that irrespective of the boundary conditions, the transition from no structure (gray) to any structure (magenta and cyan) is approximately the same. This observation suggests that the critical magnetic strength required for cluster formation $\mu^{*}$ is not influenced by the boundaries of the system. Indicating that this mechanism of structure formation is not strongly affected by the geometry and wall induced torques, but the relative frequency of chain and ring formation is affected.

Changing the geometry of the confinement while keeping the boundary conditions purely repulsive $(\alpha=0.0)$ seems to have the biggest impact on structure formation of dipolar particles. Here, we observed formation of chains (cyan area) in the circular confinement for active particles with high motility $\left(v_{0}=40\right)$. The absence of corners in the circular environment removes the possibility for an already formed chain to get stuck in a corner and catch its own tail to form a ring. Hence, we observed fewer ring structures in a circular environment than in a squared one. Increasing the wall torque $(\alpha=0.4$ and 2.0) has no strong effect on structure formation in a squared confinement (top row). Yet, the diagram of states changes qualitatively with increasing wall torque for the case of the circular confinement (bottom row) where rings become more predominant with increasing 


\subsection{Influence of confinement on structure formation}

wall torque. For large induced wall torques $(\alpha=2.0)$ a clear separation of ring and chain states can no longer be made in the case of circular confinement (hatched area).

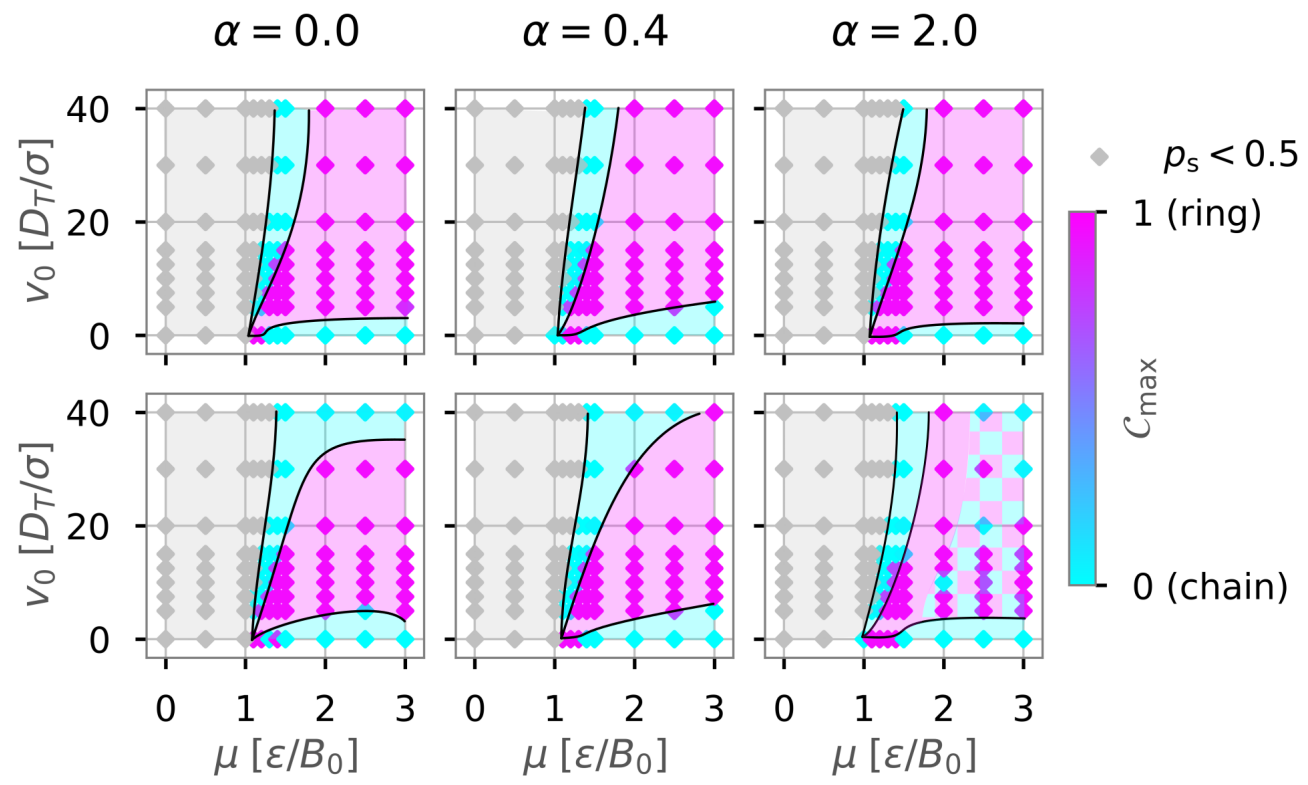

Figure 4.10.: Influence of the boundary conditions on the diagrams of states for $N=36$ particles: The shape of the confinement (first row box $L=50$, second row circle $R=50 / \sqrt{\pi}$ ) and the interactions with the walls (columns left to right: $\alpha=0.0,0.4$ and 2.0) were varied and the diagram of states determined as in Figure 4.6. The colored areas and the lines were added as visual aids. The hatched area indicates that no clear assignment to a dominant structure could be made for these parameters. Top left diagram shows same data as Figure 4.6

To gain more information about this hatched area, we took a closer look at the histograms of the order parameter for a circular confinement with a wall torque parameter $\alpha=2.0$ (see Figure 4.11). Here, we observed maxima of chain configurations with $C_{\max } \neq 0$ as well as bimodal distributions with two peaks for chain and ring configurations of approximately the same height $\left(v_{0}=20, \mu=2.5\right)$. These observations can explain the heterogeneity of the order parameter in the hatched area. 
4. Structure formation in small systems

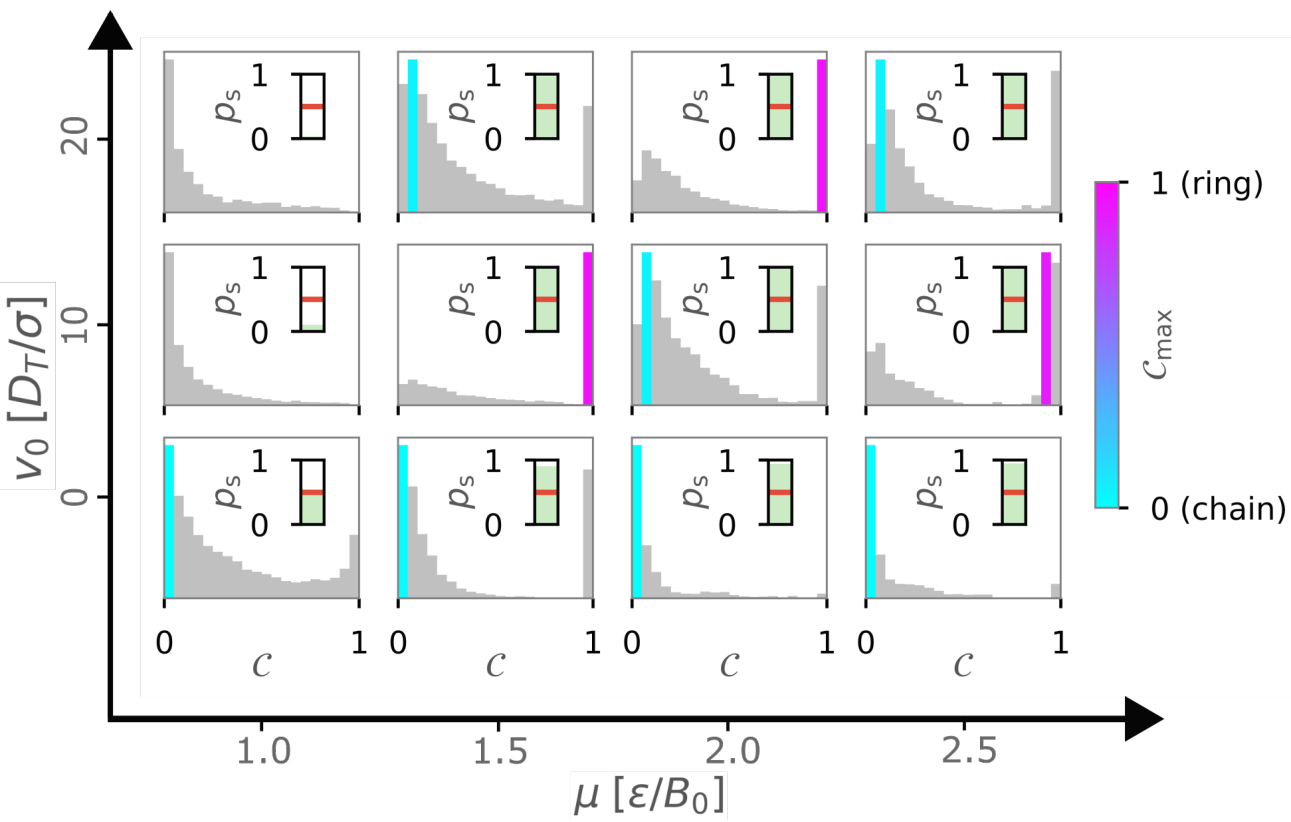

Figure 4.11.: Overview of the state weighted histograms of the order parameter $C$ for circular confinement with $R=50 / \sqrt{\pi}$ and $\alpha=2.0$ for $N=36$ particles. The self-propulsion velocity $v_{0}$ and the magnetic strength $\mu$ are varied. The color bar indicates the dominant order parameter $C_{\max }$ corresponding to a chain (cyan) or ring (magenta) configuration. As in Figure 5, if probability of structure formation $p_{\mathrm{s}}<0.5$ (inset) all bars remain gray. Data obtained from 10 independent simulations.

\subsubsection{Influence of density in confinement}

In this section we discuss how density affects cluster formation. We modulated the density by increasing the particle number, as well as by reducing the size of the simulation box. So far all the simulations were done for $N=36$ particles. Now, we ask how increasing the density by increasing the particle number affects structure formation. To answer this question, we increased the particle number to $N=81$ particles with a box size of $L=50(\Phi=0.03)$ for different geometries and wall interactions. For a systematic analysis, we repeated the calculation of the order parameter $C$ and determined diagrams of states (see Figure 4.12 ) as before. 


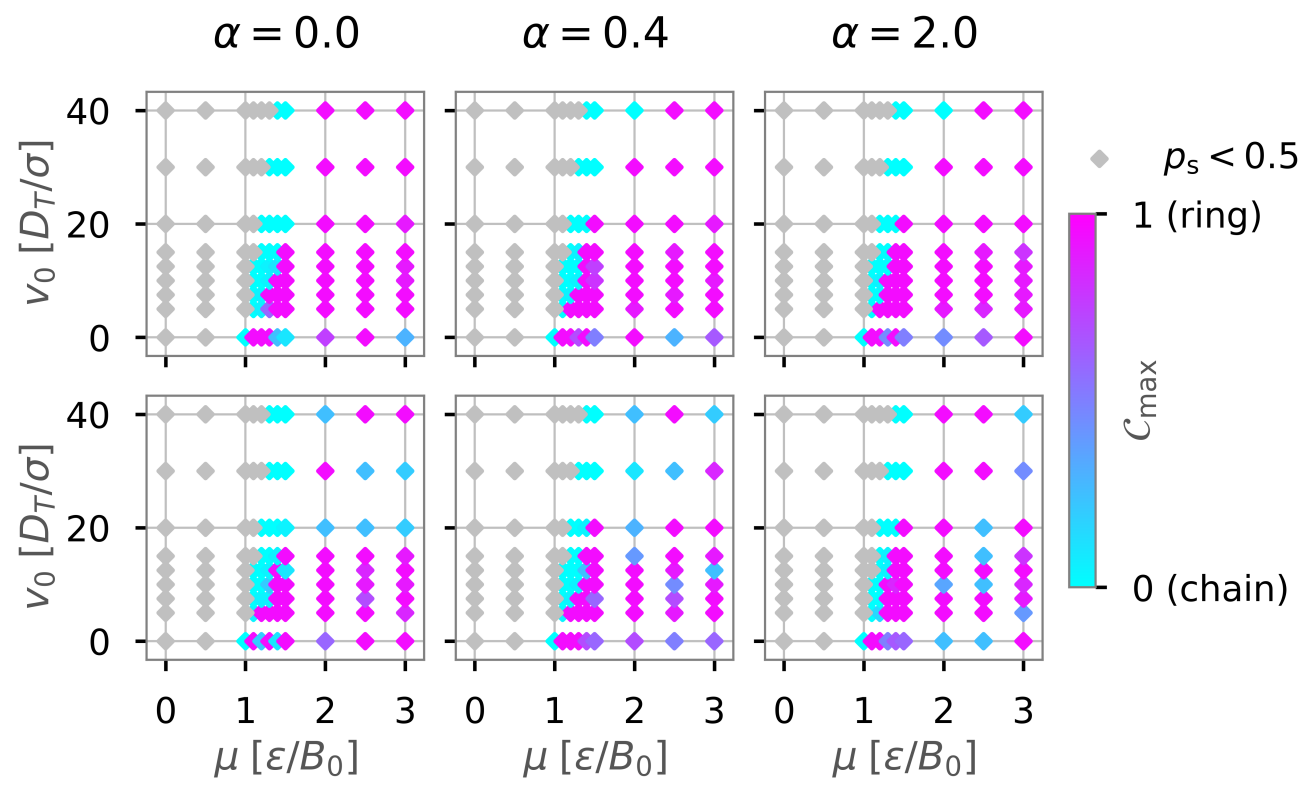

Figure 4.12.: Diagram of states according to the order parameter $C_{\max }$ for $N=81$ particles and different boundary conditions (top row: box confinement $L=50$, bottom row: circular confinement $R=50 / \sqrt{\pi}$, columns: different values of wall torque parameter $\alpha$. The color bar represents the order parameter.

We noticed that the diagram of states are similar to the simulations with $N=36$ particles (see Figure 4.10). Specifically, the transition from no structure (gray) to any structure (cyan and magenta) is approximately the same. However, there are differences in the regions with structures. We no longer see a clear chain regime for the passive case $\left(v_{0}=0\right)$, instead the $C_{\max }$ values indicate more heterogeneous structures. Inspection of the histograms of the order parameter $C$ (Figure 4.13) shows that for higher particle number the histograms are multi-modal and that maxima can occur at intermediate values of $C$ (cf. $\mu=2.0$ and $v_{0}=0$ ). These observations suggest that also intermediate structures like bend chains or almost closed rings can be present. However, we noticed that next to the previously seen chain and ring structures more complex structures, such as branches or loops 


\section{Structure formation in small systems}

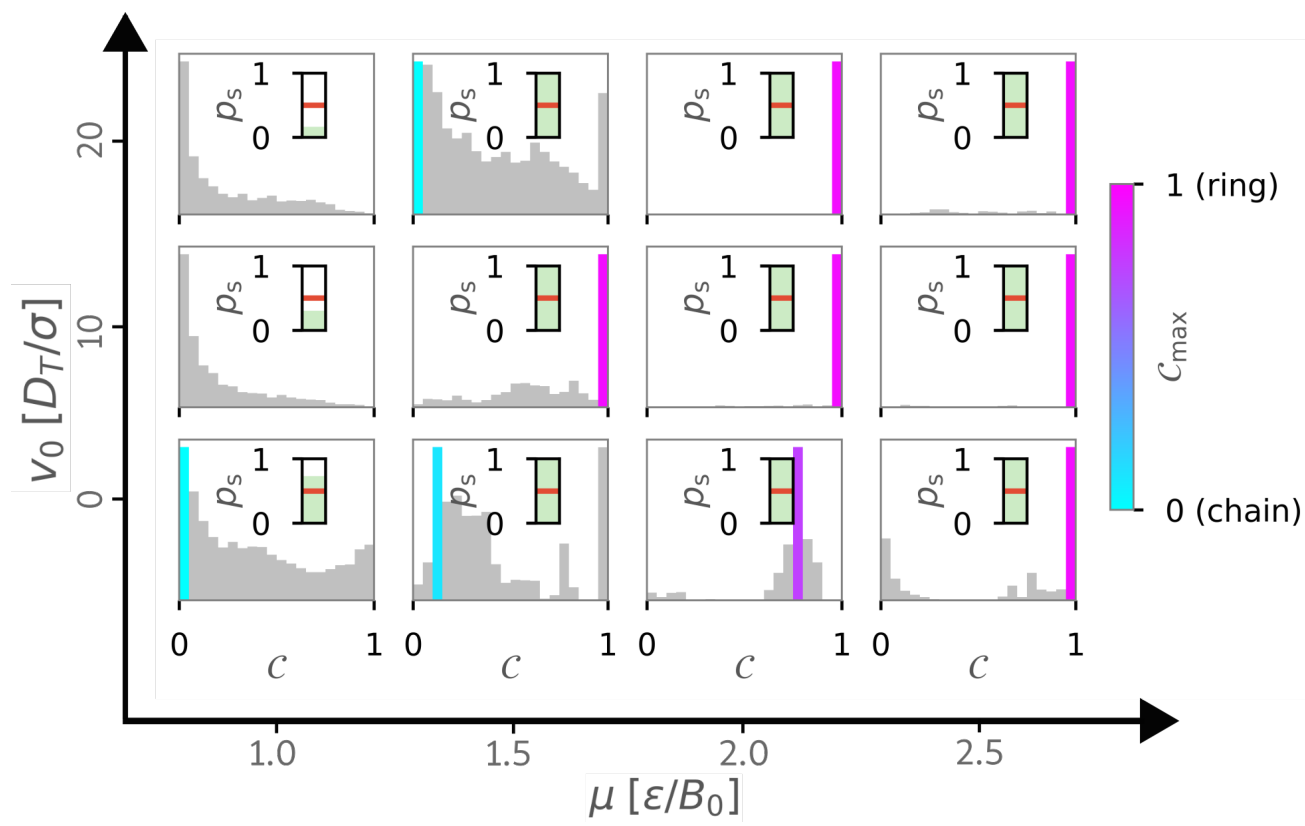

Figure 4.13.: State weighted histograms of the order parameter $C$ for $N=81$ particles in squared confinement with $L=50$ and $\alpha=0$ for varying self-propulsion velocity $v_{0}$ and the magnetic strength $\mu$. The color bar represents the dominant order parameter $C_{\max }$, corresponding to a chain (cyan) or ring (magenta) configuration. As in Figure 5, if $p_{\mathrm{s}}<0.5$ (inset) all bars remain gray.

within a ring can emerge in simulations with $N=81$ particles. Selected examples for these complex structures are shown in Figure 4.14. Such structures are also known from systems of passive particles [41] and indeed they appear to be more frequent for values of $v_{0}=0$, as indicated by the higher occurrence of intermediate values of $C$ in the histograms (see Figure 4.13). However, some of these complex structures cannot be properly characterized by our order parameter $C$, since the head-tail distance is no longer well defined, and other ways of characterizing them have to be developed.

In addition, we modulated the density by reducing the box size of the squared confinement with $N=36$ particles and purely repelling walls $(\alpha=0)$ to $L=33.3$ $(\Phi=0.03$, matching the density of the system with $N=81, L=50)$ and $L=16.8$ 


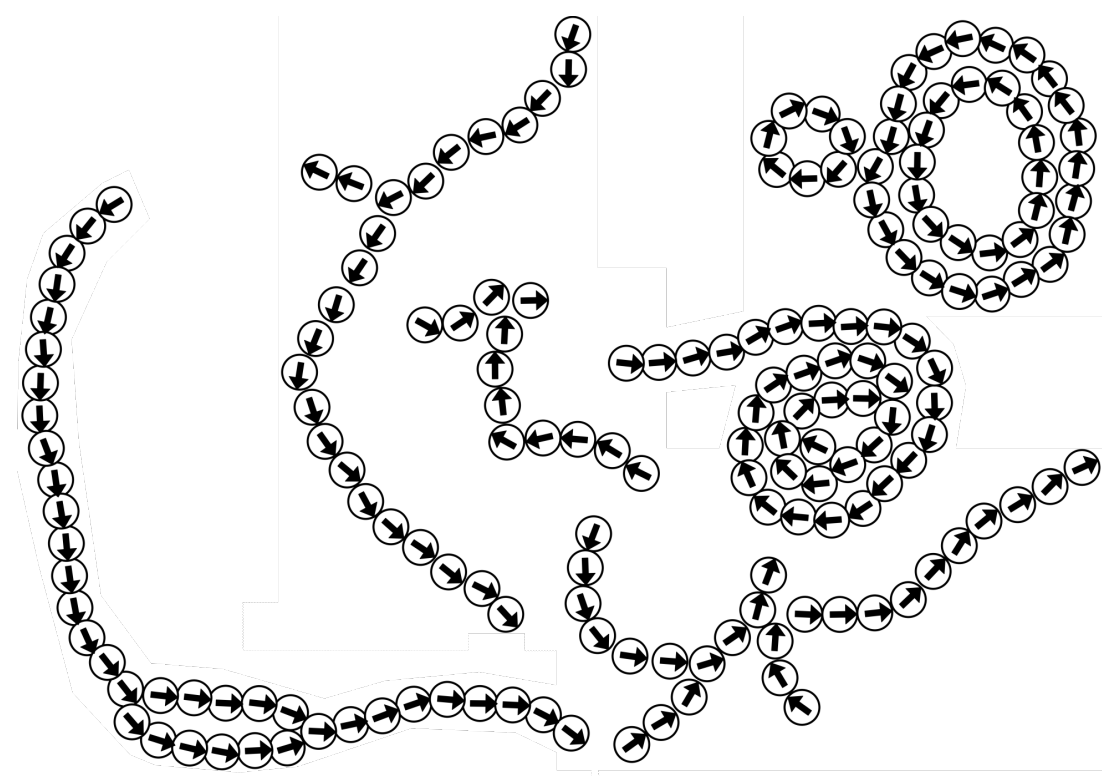

Figure 4.14.: A selection of complex structures that were observed in systems with $N=81$ particles (with different self-propulsion velocities and magnetic strengths). The direction of the magnetic moment is indicated by a black arrow. Branches, chains with loops and rings with multiple loops or multiple rings in one structure are shown.

$(\Phi=0.1)$ respectively. Figure 4.15 shows how the diagram of states (a.) and the critical magnetic strength $\mu^{*}$ for structure formation (b.) are affected by the reduced box size. In smaller systems (with increasing density) ring structures become dominant for passive particles $\left(v_{0}=0\right)$ as predicted in [53]. This result is very similar, but not exactly the same as for the system with $N=81$ particles and matching density (see Figure 4.12 top left, where differences between the two realizations of the same density are seen for small $v_{0}$ ). Furthermore, chain structures become dominant at high motilities for $\mu<1.0$ in the case of $\Phi=0.10$. This finding is in agreement with the recent results of Liao et al., who showed that the probability of chain formation is increased because of smaller mean separation between the particles for higher densities $[73]$. This explanation is confirmed by the 


\section{Structure formation in small systems}

observed shift of the critical magnetic strength with increasing density (Figure 4.15 b).

a.

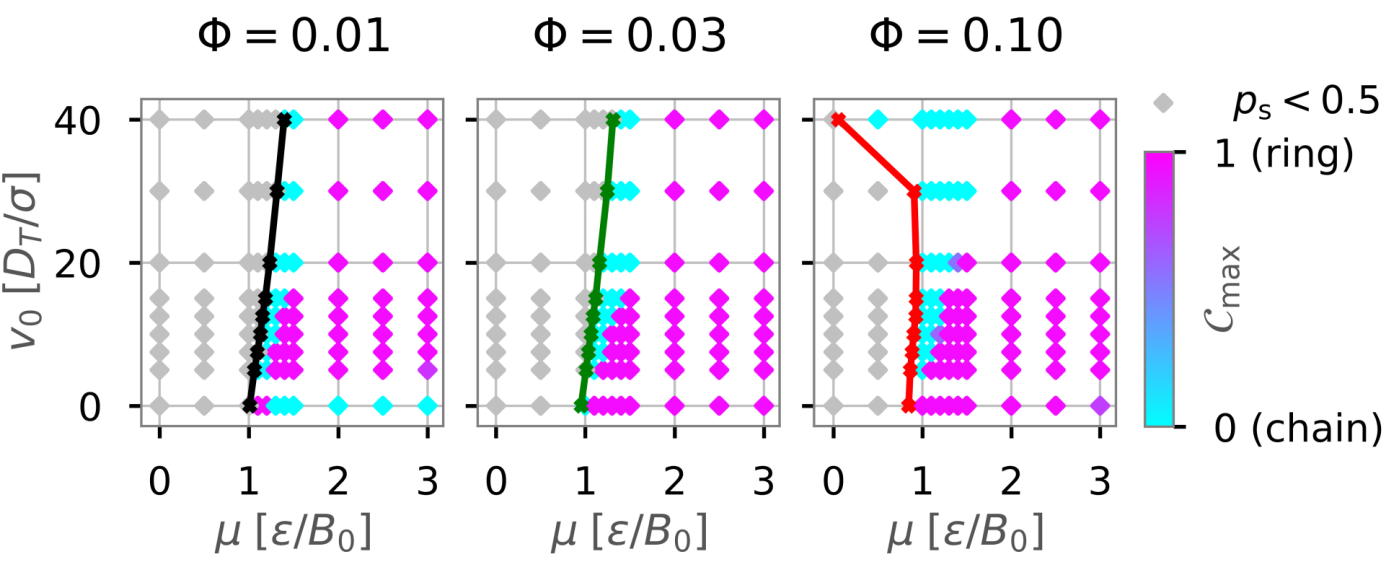

b.

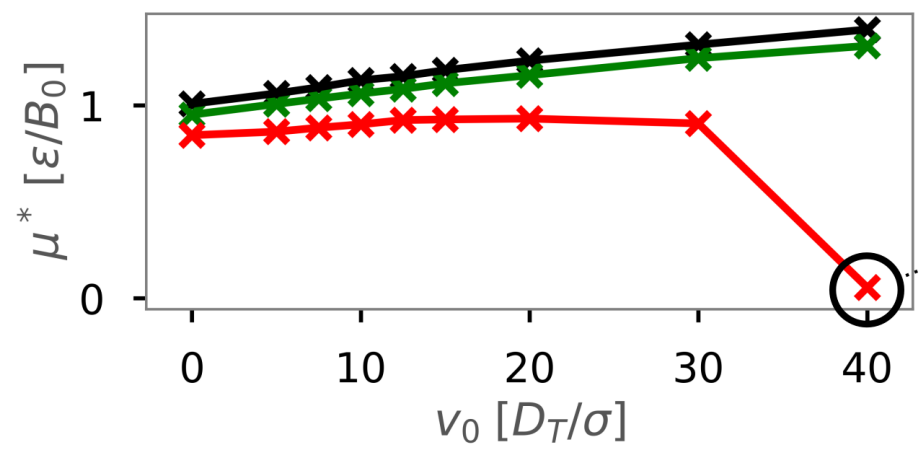

c.

Figure 4.15.: a. Diagram of states according to the order parameter $C_{\max }$ for $N=36$ particles in squared confinement with $\alpha=0$ for different densities $\Phi$ by reducing the box size $L$. The colored line shows the critical magnetic strength in the diagram of states. Data taken over 10 independent simulations. Top left diagram shows same data as Figure 4.6. b. Critical magnetic strength $\mu^{*}$ against the self-propulsion velocity $v_{0}$. The colors indicate the different densities $\Phi=0.01$ ( $L=50$, black $), \Phi=0.03$ ( $L=33.3$, green $)$, and $\Phi=0.1(L=16.8$, red $)$. The color bar represents the order parameter. c. Extract from a snapshot showing a disordered cluster formed in the top right corner of the squared confinement for the parameters $\mu=0, v_{0}=40$.

A striking deviation from this linear behavior is seen for high motility in the smallest system $(L=16.8, \Phi=0.10$, red line) where clusters are formed with- 


\subsection{Comparison to analytical solution}

out magnetic interactions $\left(\mu^{*} \approx 0\right)$. This deviation occurs when the persistence length of the active particles is much greater than the dimensions of the confining walls $v_{0} / D_{\mathrm{R}} \gg L$. Here, the formed clusters are qualitatively different from the previously observed chains and rings (see Figure 4.15 c). These particles form rather disordered, finite-sized clusters at the walls of the squared confinement $(\alpha=0)$. The clusters are similar to clusters observed for active systems that display motility-induced phase separation (MIPS) [116, 117]. In our system, the presence of boundaries seems to take the role of high density clusters during MIPS. We note that the system densities presented in this chapter are well below the critical density where MIPS is expected [118]. It was shown recently that MIPS is repressed by strong dipolar interactions between particles for larger systems with higher densities 73 .

\subsection{Comparison to analytical solution}

When comparing all presented diagrams of states, we noticed that the critical magnetic moment for cluster formation $\mu^{*}$, which is reflected in the transition from no structure (gray) to any structure (cyan and magenta), is approximately the same for all systems. In particular the slope of the linear dependence on activity (Figure 4.15, b.) is not affected. This linear dependence Figure 4.3 suggests a correction to the critical magnetic coupling strength which is linear in first order $\mu^{*}=\sqrt{T}\left(1+\gamma v_{0}\right)$ with a constant $\gamma$. Such a correction is obtained quite generally if the balance condition $\lambda=1$ or $\mu^{* 2}=T$ is modified to $\mu^{* 2}=T+f\left(v_{0}\right)$, where the second term (with $f^{\prime}(0)=\gamma$ ) describes the additional 'noise' due to active motion. As previously done in other studies [119, 120], the addition of activity can be 


\section{Structure formation in small systems}

interpreted as an effective increase in temperature. Following this interpretation, we used this additional term to re-scale the magnetic interactions in an active system to an effective magnetic interaction $\mu_{\mathrm{a}}$ in a passive system

$$
\mu_{\mathrm{a}}=\frac{\mu}{1+\gamma v_{0}}
$$

Fig. 4.16 shows the probability for cluster formation against the effective magnetic interaction strength $\mu_{\mathrm{a}}$. All curves collapse on the curve corresponding to the passive case $\left(v_{0}=0\right.$, blue), indicating that a re-scaled magnetic strength can be used to characterize cluster formation in an active system.

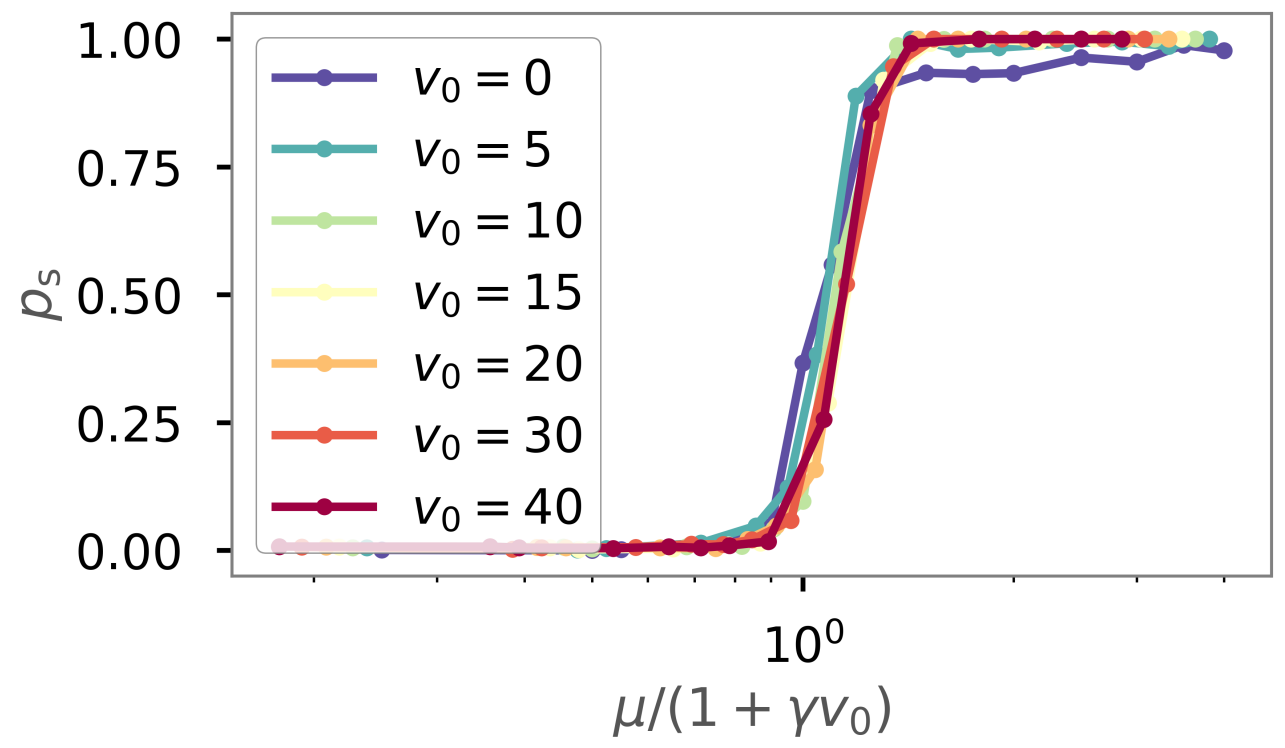

Figure 4.16.: Semi-logarithmic curves of the probability of structure formation $p_{\mathrm{s}}$ as a function of the re-scaled magnetic strength $\mu_{\mathrm{a}}$ for $N=36$ particles. Data taken from 10 simulation runs with $L=50$ (pbc). Value for $\gamma=0.013$ was obtained via least-squares fit of data presented in inset of Figure 4.3 .

In a next step, we used this re-scaled magnetic moment $\mu_{\mathrm{a}}$ to predict ring and chain formation in small systems of active dipolar particles. To this extent, we 


\subsection{Comparison to analytical solution}

used the free energy expression for ring and chain formation in systems of passive dipolar particles derived by Kantorovich et al. [53]. Here, the authors present an analytical formalism for the expected ratio between rings $n_{r}$ and chains $n_{c}$ in a system with size $N$ when minimizing the free energy of the system. Using the relations $\mu=1 / \sqrt{T}$ and $\mu_{\mathrm{a}}=\mu /\left(1+\gamma v_{0}\right)$, we rewrote the ring to chain ratio as an equation of the re-scaled magnetic moment $\mu_{\mathrm{a}}$ :

$$
\frac{f_{\mathrm{r}}}{f_{\mathrm{c}}}=\frac{q\left(\mu_{\mathrm{a}}\right)^{R(n)-C(n)}}{n^{3 \nu+1}}
$$

where

$$
\begin{aligned}
q\left(\mu_{\mathrm{a}}\right) & =\frac{1}{3 \mu_{\mathrm{a}}} \exp \left(2 \mu_{\mathrm{a}}^{2}\right) ; \\
R(n) & =\frac{n}{2} \sin ^{3} \frac{\pi}{n}\left(\sum_{k=1}^{[(n-1) / 2]} \frac{\cos ^{3}\left(\frac{\pi k}{2}\right)+1}{\sin ^{3} \frac{\pi k}{n}}+\mathrm{R}_{(n+1) / 2}\right) ; \\
C(n) & =\sum_{k=1}^{n} \frac{n-k}{k^{3}},
\end{aligned}
$$

with $\mathrm{R}_{(n+1) / 2}$ standing for the residual of division. The scaling exponent was set to $\nu=3 / 4$ in two dimensions, according to [121]. Here, $q(\mu)$ describes the pairwise energetic contributions between direct neighbors of particles in a headtail configuration, while $n^{3 \nu+1}$ accounts for the entropic contributions arising from $n$ ways opening a ring to form a chain. The term $R(n)$ stems from the partition function of a ring and the term $C(n)$ stems from the partition function of chain [53].

Based on equation 4.1, we constructed the diagram of states shown in Fig- 


\section{Structure formation in small systems}

ure 4.17 (left) for $N=36$ particles by classifying the three states as follows. If $\mu_{\mathrm{a}}<\mu^{*}$, no structures form (gray). If $\mu_{\mathrm{a}}>\mu^{*}$ and $f_{\mathrm{r}} / f_{\mathrm{c}}<1$, chains dominate (cyan). And if $\mu_{\mathrm{a}}>\mu^{*}$ and $f_{\mathrm{r}} / f_{\mathrm{c}}>1$, rings dominate (magenta). The diagram of states based on analytical predictions is qualitatively in good agreement with the diagrams presented for small confined systems at $\Phi=0.03$ based on the order parameter $\mathcal{C}$ (Figure 4.17 , right). The analytical calculation predicts a broader chain regime compared to the numerical observations. This effect is expected since in the derivation of the free energy equation by [53], the authors assume that rings with $n<5$ particles do not form and hence not contribute towards the free energy. In our classification, we assume that rings with $n>3$ can form.
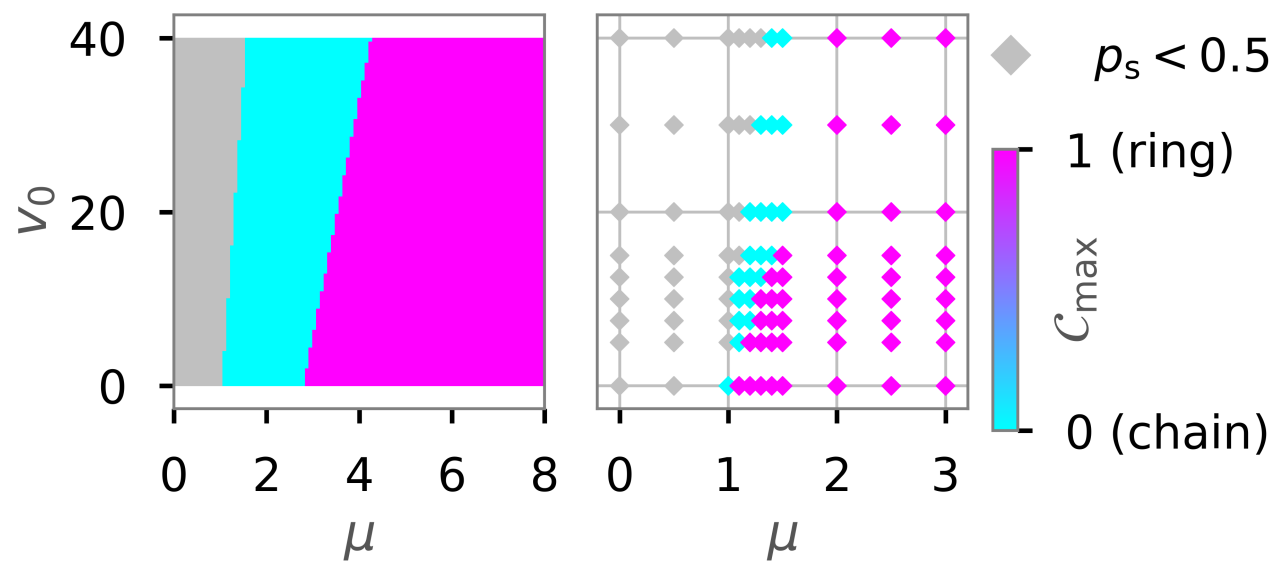

Figure 4.17.: Side by side comparison between diagram of states based on equation (4.1) (left) and simulation data of $N=36$ particles in squared confinement at density $\Phi=0.03$ (same data as Figure 4.15 a., center). States are color coded according to color bar.

Surprisingly, we have better agreement of analytical predictions with systems with squared confined than with systems without confinement (Figure 4.6). We want to emphasize that equation (4.1) does not take confining walls into account. We explain this apparent discrepancy by visually inspecting the average cluster 


\subsection{Comparison to analytical solution}

size in our simulations. Because of the increased probability density at confining the walls (Figure 4.7), we observed that systems with boundaries form bigger clusters than systems with periodic boundary conditions during our finite simulation time. This explanation is in line with the fact that confined systems at lower density $\Phi=0.01$ have a mismatch for passive particles, as presented earlier in section 4.1.1. We expect a better agreement between numerical simulations and analytical predictions if we extend our simulations of passive particles to much longer times, as demonstrated in $[53$. The fact that our results do not match quantitatively indicates the limits of interpreting activity as an effective temperature. It has been shown in other studies that the calculation of an effective temperature can be affected by density, collective phenomena and anisotropy in the system $[120,122,125]$. This dependency on the choice of observables used for re-scaling limits the predictive power of this interpretation. 


\section{Collective dynamics in large systems without external field}

In this chapter, we studied the collective dynamics in large systems of dipolar active particles without an external magnetic field. In particular, we investigated how activity and density affects the collective dynamics of active dipolar particles. To characterize the system, we calculated three order parameters, as introduced in section 2.3. fraction of largest cluster, global polarization and degree of polymerization. We present the results for four representative values for the magnetic moment of the particles in detail: apolar $(\mu=0$, black $)$, weak $(\mu=1$, blue), intermediate ( $\mu=1.4$, green) and strong $(\mu=2.6$, red). Finally, we summarize our results in diagrams of states.

To that end, we performed Brownian dynamics simulations of $N=1156$ particles in a simulation box with periodic boundaries while varying the magnetic moment and self-propulsion speed of the particles. We performed these simulations at three representative densities $\Phi=0.13, \Phi=0.23$ and $\Phi=0.57$ (see chapter 2). 


\subsection{Low density $\Phi=0.13$}

First, we studied the collective dynamics at low density $\Phi=0.13$, well below the critical density where MIPS is expected in systems of active Brownian particles. Fig. 5.1 shows typical configurations of our system at low average density of $\Phi=0.13$, intermediate self-propulsion speed $v_{0}=23$ for three different magnetic strengths $\mu$. Here, we observed a clear dependence of structural configurations on the magnetic strength. While particle orientations (color wheel) and positions are fairly disordered for weak magnetic interactions $(\mu=1.0)$, similar to a gaslike state, particles start to aggregate into short chains of various lengths with coexisting monomers for intermediate magnetic interactions $(\mu=1.4)$. For strong magnetic interactions $(\mu=2.6)$, long chains spanning the simulation box with multiple branching points and defects form.
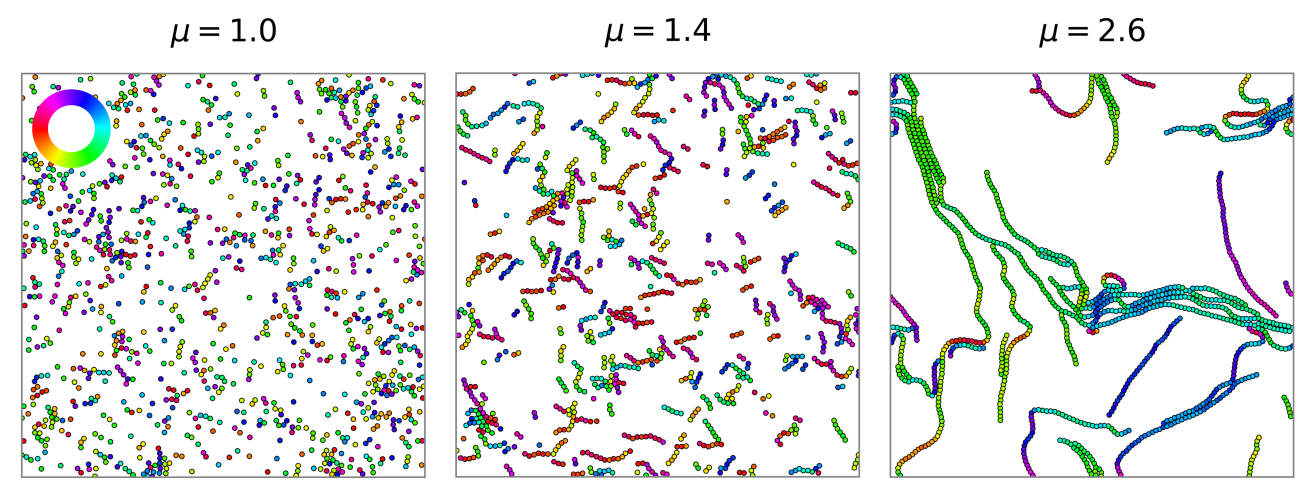

Figure 5.1.: Snapshots of systems at low density $(\Phi=0.13)$ with $v_{0}=23$ for different magnetic interactions strength $\mu$ at time $t=20$. Orientation of the particles is indicated by the color wheel (top left).

As demonstrated by Figure 5.1, the system usually contains chains of various sizes $n$. Therefore, we ask the question, what is the probability that a randomly selected particle belongs to a chain of size $n$ ? Fig. 5.2 shows the distribution of 
5. Collective dynamics in large systems without external field

the chain sizes $P(n)$ weighted by the chain length $n$ for low $(\mu=1.0)$, intermediate $(\mu=1.4)$ and strong magnetic interactions $(\mu=2.6)$ for four different self-propulsion speeds $v_{0}$. In the case of low magnetic interactions (left), the distribution follows a power-law behavior, as has been theoretically predicted for passive systems with non-interacting dipolar chains 126 . Here, the passive case $\left(v_{0}=0\right.$, blue circles) recovers the predicted behavior for $n \geq 10$. In the case of active particles the power-law exponent depends on the self-propulsion speed and magnetic interaction strength $\mu$, as has been observed by others 73 .

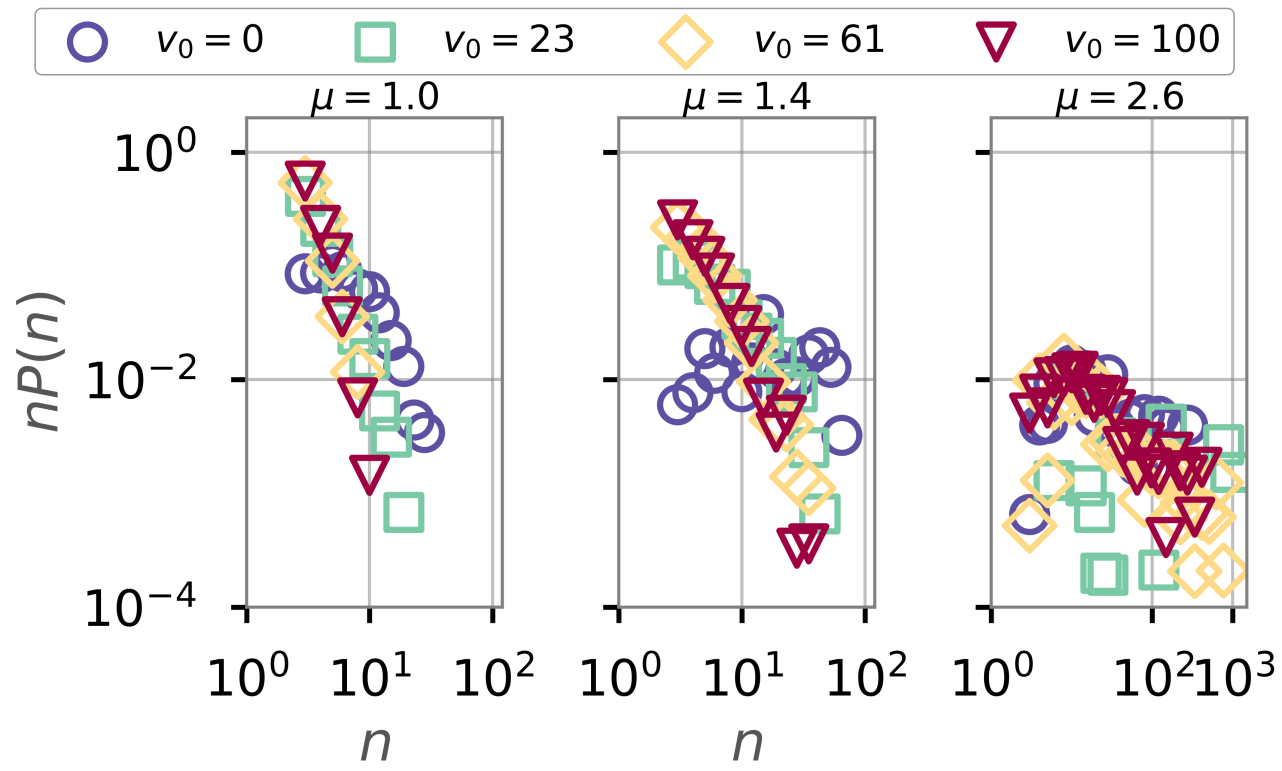

Figure 5.2.: Log-log plot of probability distribution of chain sizes $P(n)$ weighted by number of monomers $n$ per chain for different self-propulsion speeds $v_{0}$. Distributions for systems with low $(\mu=1.0$, left), intermediate $(\mu=1.4$, center $)$ and strong magnetic interaction strength $(\mu=2.6$, right) are shown. Average system density is set to $\Phi=0.13$. Cut-off for cluster formation was set at $\epsilon=1.28$. Data taken from $t=18$ to $t=20$ every $\Delta t=0.1$.

For intermediate magnetic interactions $\mu=1.4$ (center), the chain size distribution does no longer follow a power-law behavior for passive particles $\left(v_{0}=0\right.$, 
blue circles). Here, passive chains collide with each other and form small-scale clusters as measured by the order parameter $\left\langle\phi_{c}\right\rangle \approx 0.2$ (Figure 5.3 , top). The appearance of these clusters defies some assumptions made in [126]. Here, it is assumed that chains only interact with free monomers but not with other chains. Systems with strong magnetic interactions $\mu=2.6$ (right) show maxima in the probability distribution of chain sizes, indicating that a preferred chain size exists $\left(v_{0}=100\right.$, red triangles). This value seems to be affected by the activity of the particles.

In a next step, we quantify our observations of the collective behavior demonstrated in Figure 5.1 by calculating the three global order parameters fraction of largest cluster, global polarization and degree of polymerization, as introduced in chapter 2 . Fig. 5.3 shows the dependence on self-propulsion speed for all three order parameters at low density $\Phi=0.13$.

The fraction of the largest cluster compared to system size $\left\langle\phi_{c}\right\rangle$ (Figure 5.3, top) shows that no large scale clusters are present for dipolar active particles with low or intermediate magnetic moments $(\mu \leq 1.4)$. Only for strong magnetic interactions ( $\mu=2.6$ ) large scale clusters start to form (red curve). Here, the relative cluster size exceeds values greater than half the system size $\left(\left\langle\phi_{c}\right\rangle>0.5\right.$, dashed line for intermediate self-propulsion speeds $\left(v_{0}=4\right.$ to 38$)$. At larger values of selfpropulsion speed $v_{0}$, we observed that the relative cluster size is reduced with increasing self-propulsion speed.

The appearance of a maximum for particles with strong magnetic interactions (red curve) for intermediate self-propulsion speeds can be explained by investigating the temporal evolution of the order parameter $\phi_{c}(t)$ as shown in Figure 5.4 . In contrast to systems with lower magnetic strength (shades of gray and shades of 
5. Collective dynamics in large systems without external field

$$
\longrightarrow \mu=0.0-\mu=1.0 \quad \mu=1.4 \quad-\quad-\mu=2.6
$$
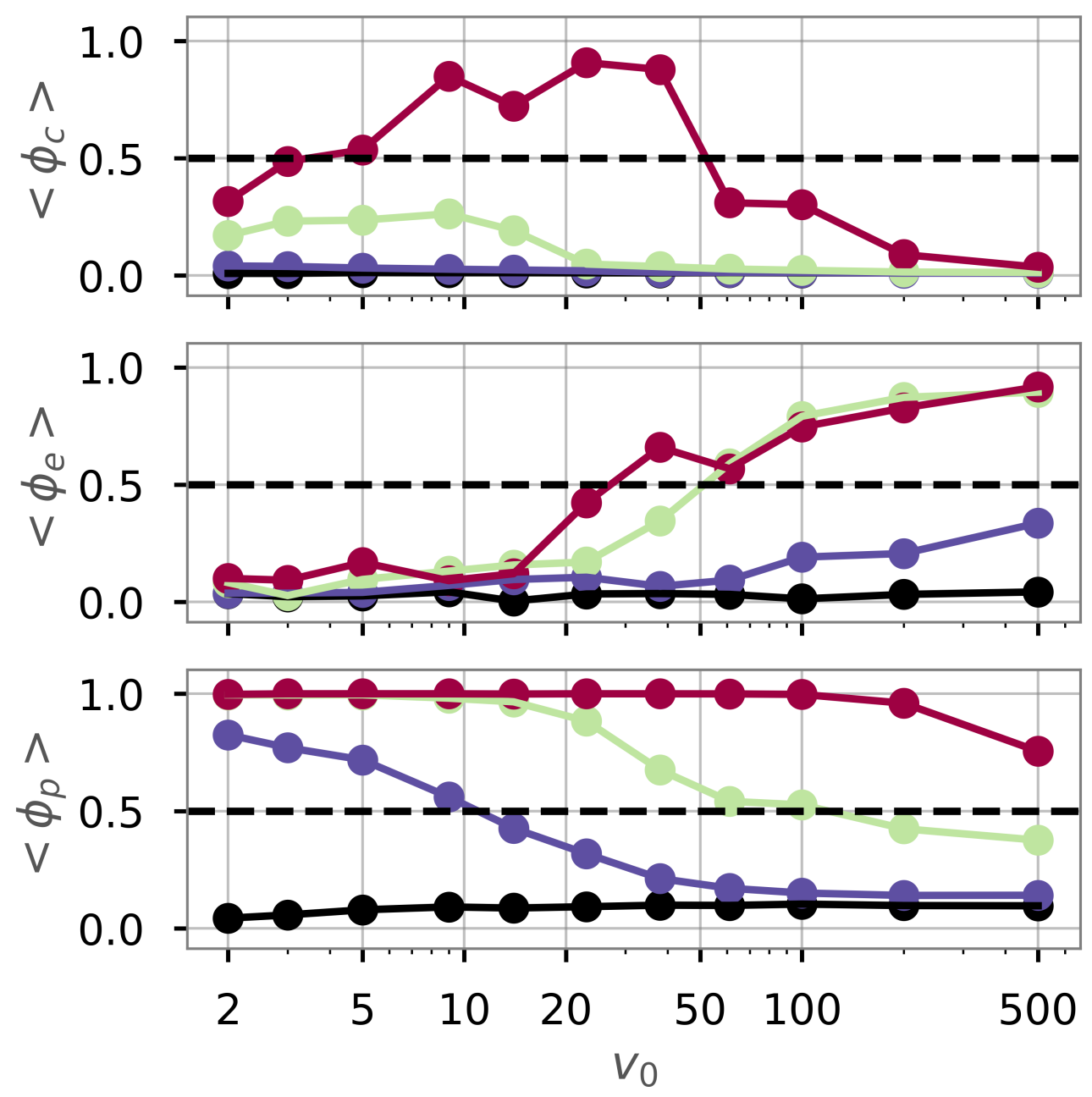

Figure 5.3.: Dependence of the three order parameters fraction of largest cluster $\left\langle\phi_{c}\right\rangle$ (top), degree of polarization $\left\langle\phi_{e}\right\rangle$ (center) and degree of polymerization $\left\langle\phi_{p}\right\rangle$ (bottom) as a function of self-propulsion speed $v_{0}$ for different magnetic interaction strengths $\mu$. System density is set to $\Phi=0.13$. Cut-off for cluster formation set at $\epsilon=1.28$ 
green), systems with high magnetic strength (shades of red) show large fluctuations of the order parameter in time, suggesting that large clusters continuously form and break up instead of single particles entering and leaving an already formed cluster. These differences in cluster dynamics were also observed in movies of the simulations. Here, long chains align with other already formed chains and briefly form a large cluster, only to separate at a later point in time.

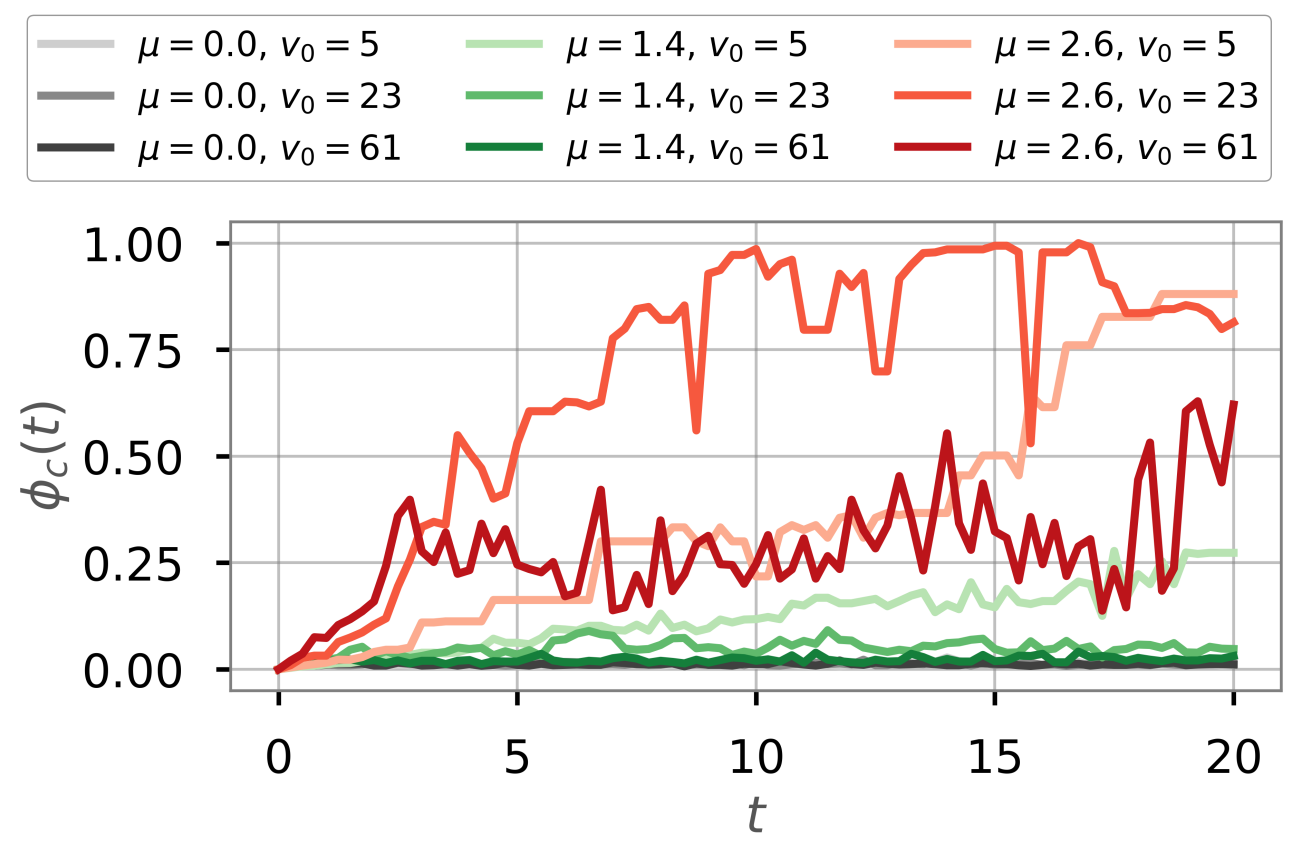

Figure 5.4.: Time dependency of fraction of largest cluster relative to system size, $\Phi=0.13$. Cut-off for cluster formation set at $\epsilon=1.28$

Calculation of the global orientation $\left\langle\phi_{e}\right\rangle$ (Figure 5.3. center) shows that the polarization of the system strongly increases with the self-propulsion speed. Even systems with weak magnetic interactions $(\mu=1.0$, blue curve) show an increase in the degree of polarization at high self-propulsion speeds $v_{0}$. This observation can be explained as follows: With higher self-propulsion speeds the time between consecutive collisions is reduced. During a collision particles align due to their 


\section{Collective dynamics in large systems without external field}

magnetic interactions and carry this alignment into the next collision, even if the magnetic interactions are not strong enough to form a bond. Multiple consecutive collisions cause orientational order to emerge spontaneously. This effect is similar to the increase of polarization with swimming speed seen in the Vicsek model 33 and has already been observed by others $[73]$. Systems without magnetic interactions ( $\mu=0$, black curve) show no orientational order as expected.

The degree of polymerization $\phi_{p}$ (Figure 5.3 , bottom) shows a strong dependence on the magnetic strength. For higher magnetic interactions larger degrees of polymerization are reached, qualitatively matching the results presented for small systems (Figure 4.3). With increasing activity, the degree of polymerization is reduced. In the case of weak magnetic interactions ( $\mu=1$, blue curve), the degree of polymerization converges towards the apolar case $(\mu=0$, black curve). For apolar particles we observed a small increase in the degree of polymerization which can be explained by an increase in collision rates: With increasing collision rates the probability of particles colliding while being briefly aligned during that collision also increases. All curves seem to eventually approach this polymerization limit set by apolar active particles.

We want to emphasize that although the degree of polymerization slightly increases for apolar particles with activity, the dynamics of these chains are vastly different from the polar case. In an apolar system these formed chains only exist briefly during the moment of collision while in a system with dipolar particles chains exist for much longer times. Visual inspection of movies of the simulations suggest that the life time of these chains depends on the magnetic interaction strength and the self-propulsion speed of the particles.

All three order parameters together indicate that dipolar particles in systems 
with low average densities $(\Phi=0.13)$ tend to organize into short chains that globally exhibit weak polar order. The actual degree of polymerization and polar order depends on both magnetic strength $\mu$ and self-propulsion speed $v_{0}$. While activity increases polar order, the degree of polymerization is greatly reduced at higher self-propulsion speeds.
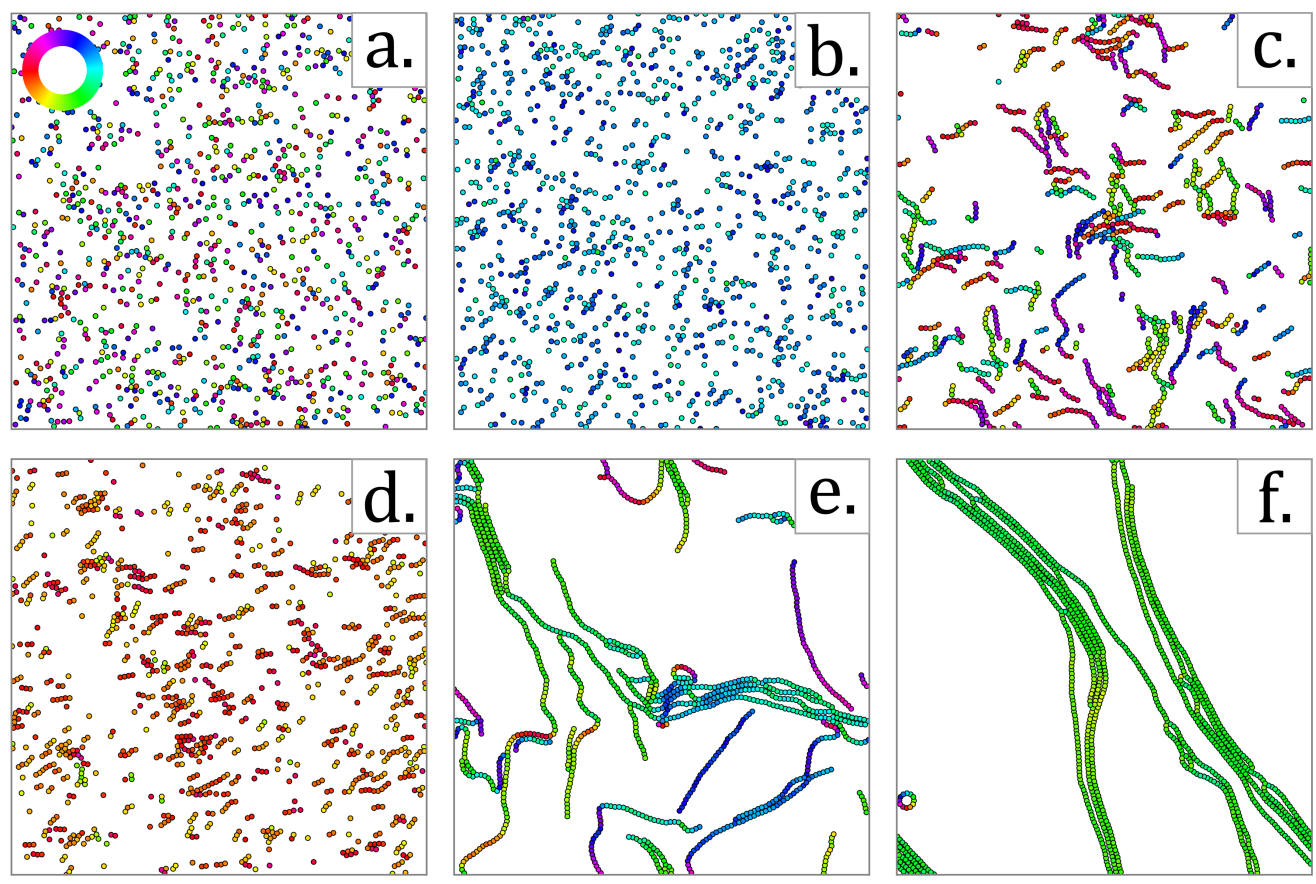

Figure 5.5.: Snapshots showing examples for states introduced in Figure 5.6 for systems at low density $(\Phi=0.13)$. Snapshots were taken at time $t=20$. The following states are shown: a. disordered gas $\left(\mu=0, v_{0}=23\right)$, b. oriented gas $\left(\mu=2.6, v_{0}=500\right)$, c. gas of chains $\left(\mu=1.6, v_{0}=23\right)$, d. oriented chains $\left(\mu=1.6, v_{0}=500\right)$, e. network of chains $\left(\mu=2.6, v_{0}=23\right)$, f. bands $(\mu=4$, $\left.v_{0}=100\right)$. The color wheel (top left) indicates the orientation of the particles.

These two opposing effects of activity lead to a rich variety in collective behavior which strongly depends on self-propulsion speed and magnetic moments of the particles. In a next step, we characterize the emerging patterns using the three global order parameters and summarize our results in a diagram of states. Based on 


\section{Collective dynamics in large systems without external field}

the classification method introduced in chapter 2 the following eight combinations are possible, each characterizing a systems' state as shown in Table 2.1 cluster, oriented gas, gas of chains, oriented cluster, network of chains, oriented chains, bands, disordered gas. Examples for each observed state at low densities are shown in Figure 5.5. We note that oriented clusters would inevitably have a high degree of polymerization in our systems. Therefore, we never observed this state in our simulations. When all three criteria are fulfilled, we see that the system develops a percolating band of chains spanning the whole system size (see Figure 5.5 f.). Overall, the states observed here are qualitatively similar to the states identified in 73 .

Fig. 5.6 shows the diagram of states for a system with low average density. Here, we observed six out of the eight possible states. For weak magnetic interactions $(\mu<1)$ no classification criterion is fulfilled and the system is in a disordered gas state (gray circle). Only for stronger magnetic interactions $(\mu \geq 1.0)$ order emerges. At this point, contributions from dipolar interactions are equal to the energy contributed by thermal noise $\mu^{2}=T=1$, which is consistent to cluster formation in small systems (see chapter 4). While varying the activity different states can be realized for fixed magnetic strengths. For example, in the case of intermediate magnetic strength $\mu=1.4$ systems with low levels of activity tend to be in a gas of chains (pink triangle). Increasing the activity adds global polarization and oriented chains (green pentagon) form. For even higher self-propulsion speeds formation of chains is suppressed while global polarization persists, we observe an oriented gas (purple plus). 


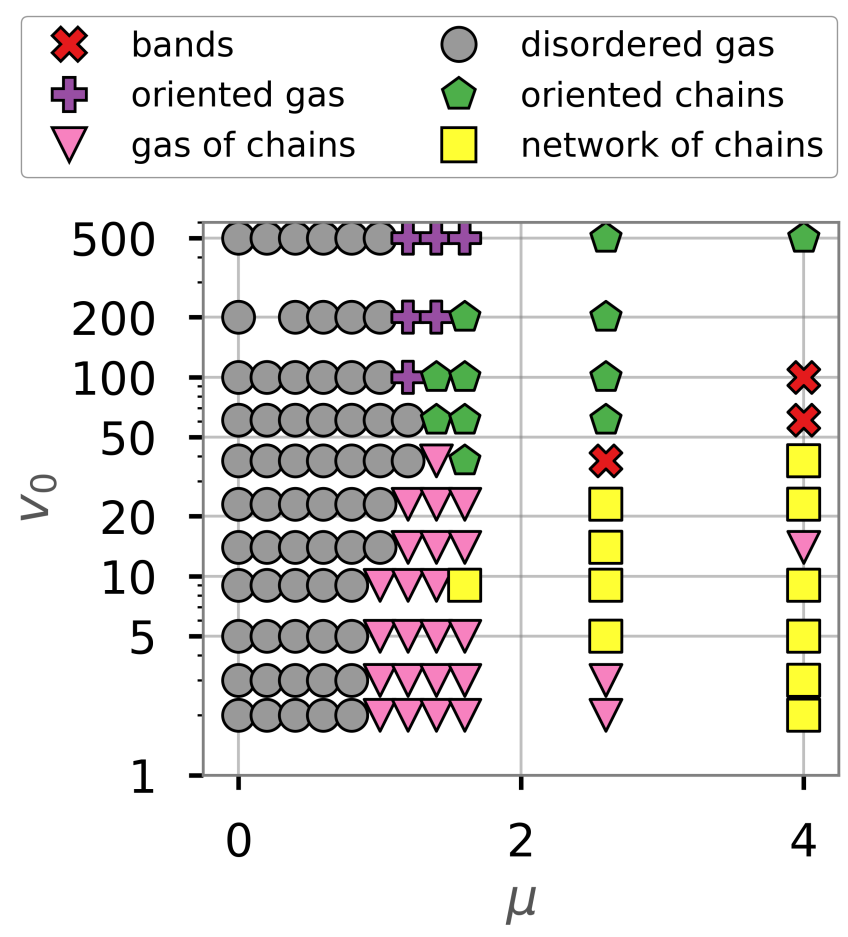

Figure 5.6.: Diagram of states for systems at low density $(\Phi=0.13)$ for different magnetic interaction strength $\mu$ and self-propulsion speeds $v_{0}$. Classification of states is based on combinations of order parameter criteria (see Table 2.1).

\subsection{Intermediate density $\Phi=0.23$}

In a next step, we studied the collective dynamics at intermediate density $\Phi=0.23$, just below the critical density where MIPS is expected systems of active Brownian particles. Figure 5.7 shows selected snapshots of the system at intermediate density $\Phi=0.23$. For low $(\mu=1.0)$ and intermediate $(\mu=1.4)$ magnetic interaction strengths, the here observed configurations are qualitatively similar to systems at low density (Figure 5.1). At high magnetic interaction strengths $(\mu=2.6)$ chains spanning the system tend to organize into broader bands with multiple lanes and defects.

A quantitative analysis of the order parameters is shown in Figure 5.8. In con- 
5. Collective dynamics in large systems without external field
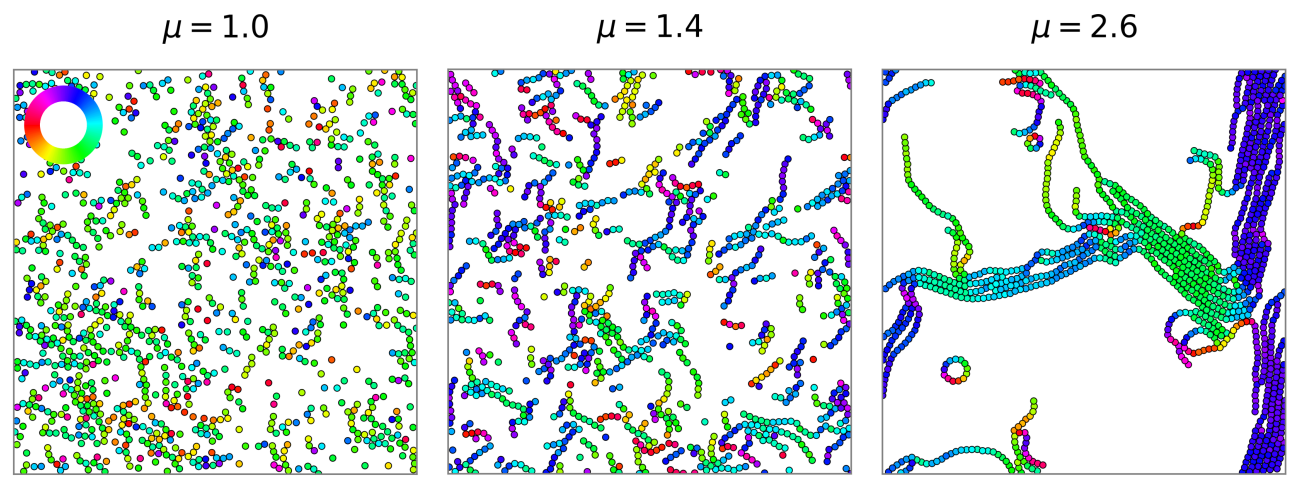

Figure 5.7.: Snapshots of systems at intermediate density $(\Phi=0.23)$ with $v_{0}=23$ for different magnetic moments $\mu$ at time $t=20$. Orientation of the particles is indicated by the color wheel (top left).

trast to systems at low density, large scale clusters form already at intermediate magnetic interaction strengths $\mu \geq 1.4$ (Figure 5.8 , green and red curves) in systems at $\Phi=0.23$. Here, the size of the largest cluster is comparable to the system size $\left(\left\langle\phi_{c}\right\rangle>0.8\right)$. Activity reduces the size of the large scale cluster to the point where $\left\langle\phi_{c}\right\rangle$ reaches values close to zero for high self-propulsion speeds. The level of activity needed to suppress large scale clusters depends on the magnetic moment of the particles. For systems with strong magnetic interactions (red curve) large scale clusters continue to exists at higher self-propulsion speeds compared to systems with intermediate magnetic strengths (green curve).

Fig. 5.8 shows the average global orientational order $\left\langle\phi_{e}\right\rangle$ for systems with intermediate density $(\Phi=0.23)$. In contrast to systems with low density $(\Phi=0.13$, Figure 5.3), the system starts to exhibit polar order already for low magnetic interactions $(\mu \geq 1.0)$. Here, the degree of polarization seems to be fairly independent of the magnetic interaction strength. Yet, we still observe a strong dependence on the activity. The system is globally polarized $\left(\left\langle\phi_{e}\right\rangle>0.5\right.$, dashed horizontal line) for self-propulsion speeds $v_{0}>20$, almost reaching total polarization $\left(\left\langle\phi_{e}\right\rangle>0.8\right)$ 

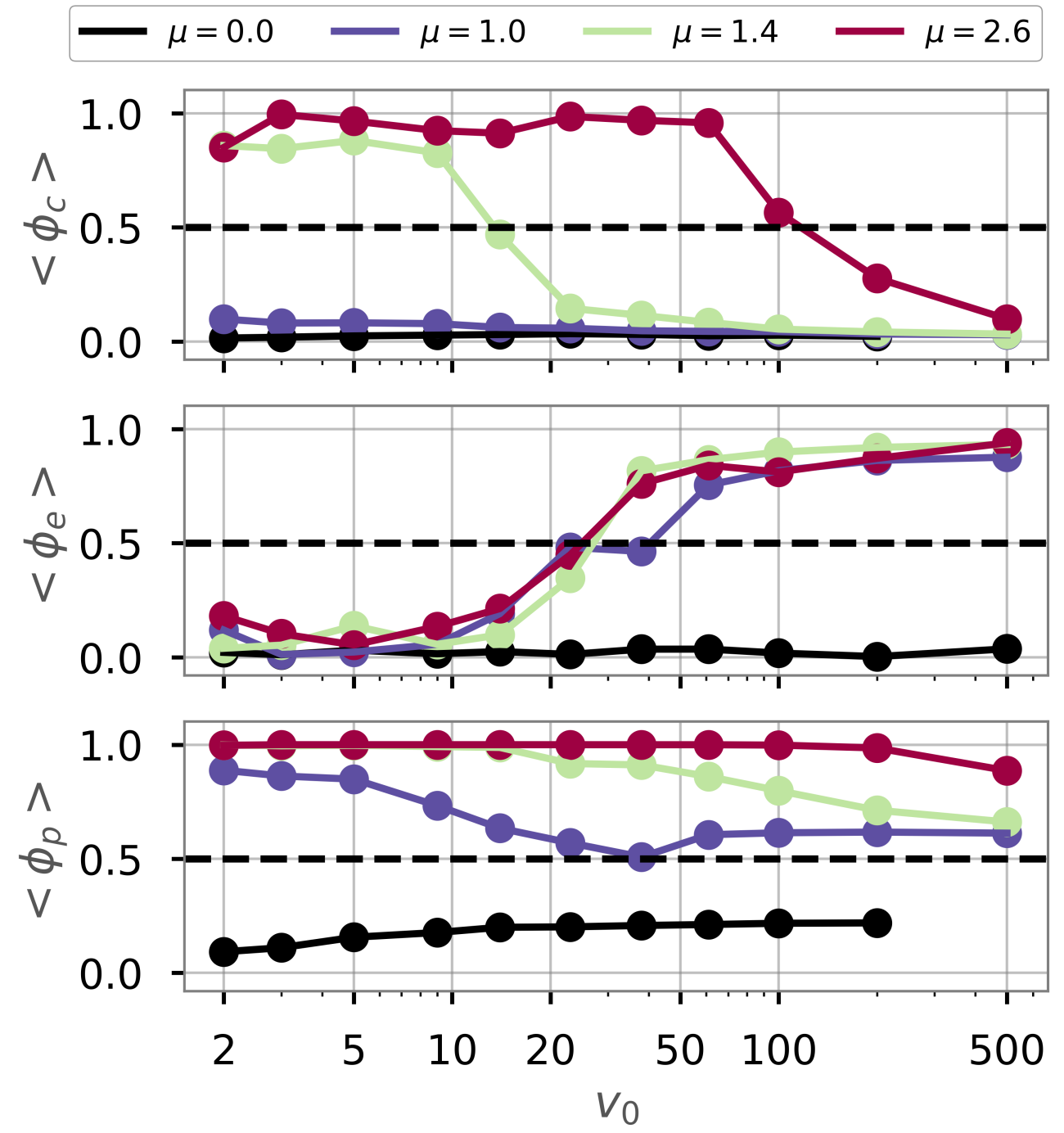

Figure 5.8.: Dependence of the three order parameters fraction of largest cluster $\left\langle\phi_{c}\right\rangle$ (top), degree of polarization $\left\langle\phi_{e}\right\rangle$ (center) and degree of polymerization $\left\langle\phi_{p}\right\rangle$ (bottom) as a function of self-propulsion speed $v_{0}$ for different magnetic moments $\mu$. System density is set to $\Phi=0.23$. Cut-off for cluster formation set at $\epsilon=1.21$. 


\section{Collective dynamics in large systems without external field}

at high self-propulsion speeds $\left(v_{0}=100\right)$. Again, systems without magnetic interactions $(\mu=0$, black curve) show no global orientational order.

Figure 5.8 bottom shows the degree of polymerization. Systems at intermediate density $(\Phi=0.23)$ are highly polymerized. Here, the degree of polymerization increases with the magnetic moment $\mu$, but decreases when activity is increased. For particles without magnetic interactions (black curve) an increase of the degree of polymerization with activity can be observed, approaching a limit of $\left\langle\phi_{p}\right\rangle \approx 0.2$ for high self-propulsion speeds. The curves of the polar particles (blue, green and red) suggest that a limit of polarization $\left\langle\phi_{p}\right\rangle \approx 0.6$ for high self-propulsion speeds independent of magnetic interactions exists that differs from the apolar case (black curve).

All three order parameters together indicate that dipolar particles in systems at intermediate average densities $(\Phi=0.23)$ tend to organize into large clusters that exhibit strong polar order globally, which are known as polar bands or lanes for high values of magnetic interactions (see Figure 5.7). Lane and band formation has been investigated in similar systems of active particles. It has been reported that anisotropy is the driving factor for band formation in active systems. The origin of anisotropic interactions is not relevant. It can either be introduced by polar interactions, like in our systems, or by shape anisotropy $72,127,128$.

The effect of an increased density is also seen in the diagram of states (Figure 5.9. An increase in density enhances all positive effects magnetic interactions and activity have on the three order parameter. Therefore, we observe overall an increase in the degree of polarization, polymerization and clustering. However, the negative effects of activity, i.e. decrease in clustering and polymerization, are diminished. As a result, we observe that disordered states (disordered gas, gas of 


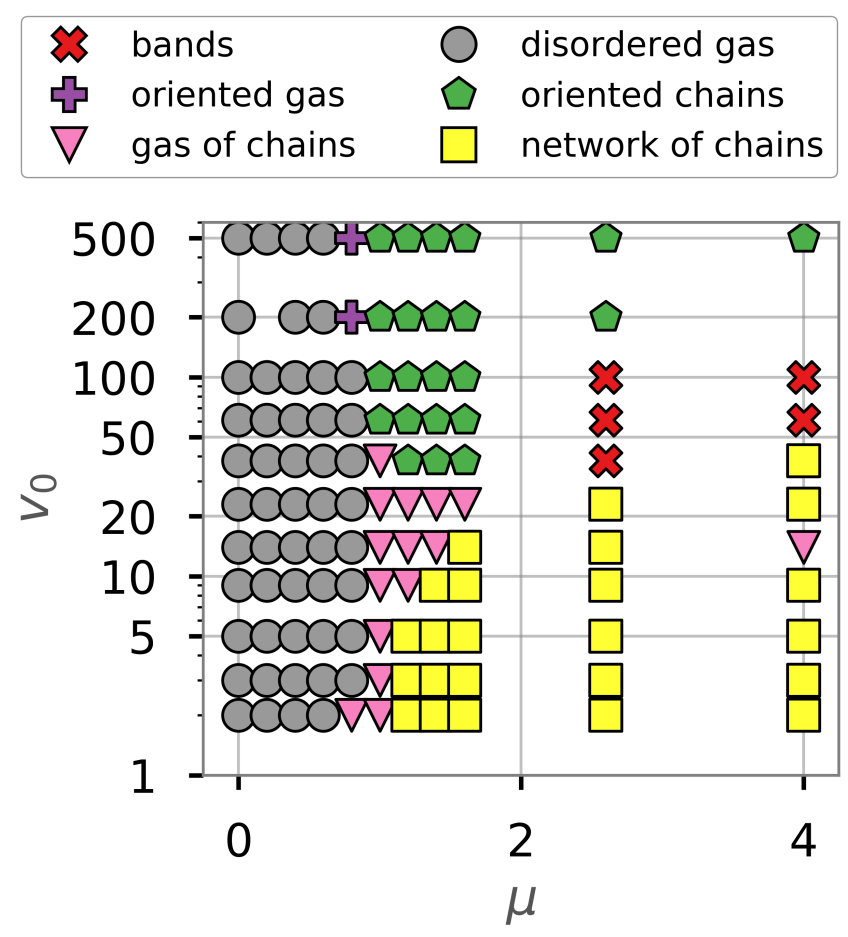

Figure 5.9.: Diagram of states for systems at low density $(\Phi=0.23)$ for different magnetic moments $\mu$ and self-propulsion speeds $v_{0}$. Classification of states is based on combinations of order parameter criteria (see Table 2.1).

chains) are suppressed in the diagram of states. In addition, oriented gas states (purple plus) seem to be suppressed at intermediate density.

\subsection{High density $\Phi=0.57$}

Finally, we studied the collective dynamics at high density $\Phi=0.57$, well above the critical density where MIPS is expected systems of active Brownian particles. Fig. 5.10 shows typical configurations of our system at high density of $\Phi=0.57$, intermediate self-propulsion speed $v_{0}=23$ for three different magnetic interaction strengths $\mu$. In contrast to systems with low and intermediate densities, particles 


\section{Collective dynamics in large systems without external field}

with low magnetic interaction strength $(\mu=1.0)$ seem to exhibit global order as indicated by the purple-blue colors of the particles. This global order persist for intermediate magnetic interactions $(\mu=1.4)$. Here, a network of orientationally ordered chains is formed. For strong magnetic interactions $(\mu=2.6)$ long chains spanning the simulation box, aligned in broad bands or lanes can be observed. Typically, alignment of chains is off-register and shows defects. Off-register alignment has been observed previously in similar systems and has been shown to be energetically favorable $39,129,130$
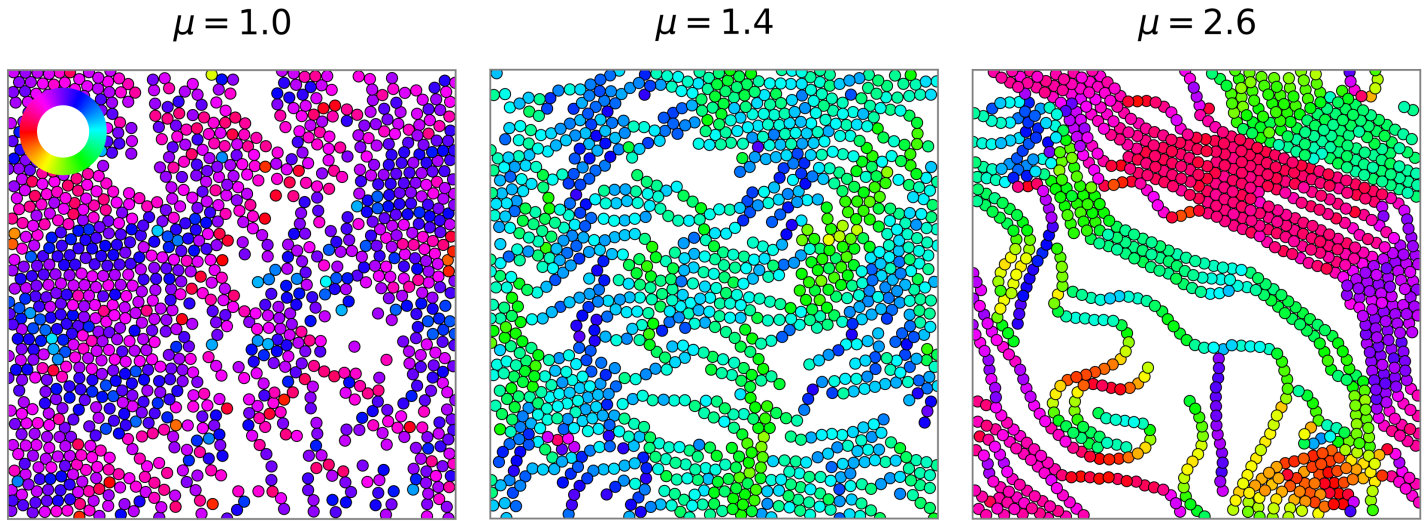

Figure 5.10.: Snapshots of systems at intermediate density $(\Phi=0.57)$ with $v_{0}=23$ for different magnetic moments $\mu$ at time $t=20$. Orientation of the particles is indicated by the color wheel (top left).

Figure 5.11 shows the fraction of the largest cluster $\left\langle\phi_{c}\right\rangle$ for systems at high density. In contrast to systems with low and intermediate densities, we observed formation of large scale clusters in systems without magnetic interactions $(\mu=0$, black curve). Here, as the self-propulsion speed $v_{0}$ increases, large scale clusters start to form. This effect is also known as motility induced phase separation (MIPS). MIPS is expected to occur in systems of active particles without attractive interactions when the average density exceeds the critical density $\Phi_{\text {MIPS }}=0.28$ 
in two dimensions $[74]$. Finite size effects $[75]$ and particle softness $[76$, among others, were shown to influence the exact value of the critical density.
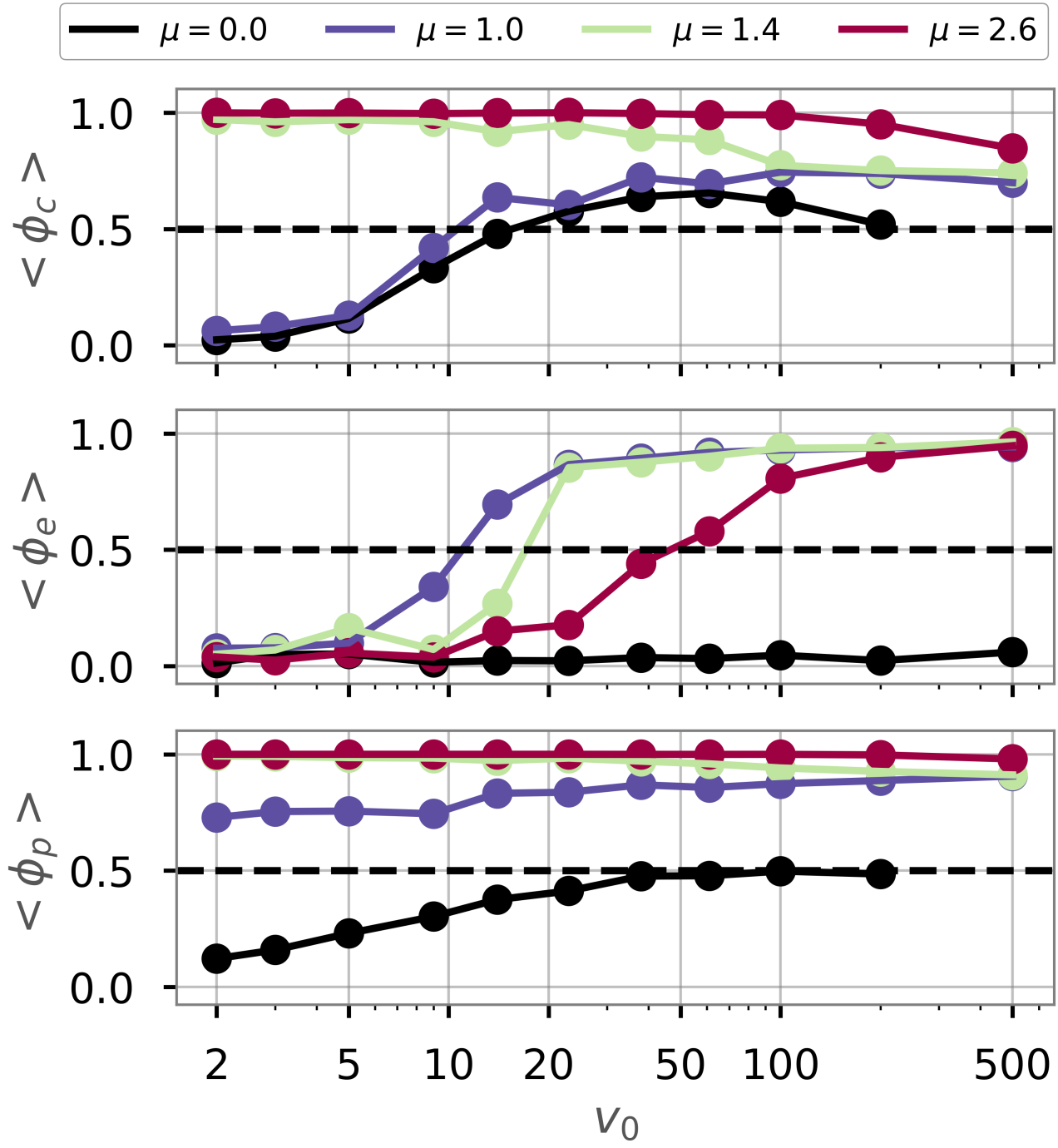

Figure 5.11.: Dependence of the three order parameters fraction of largest cluster $\left\langle\phi_{c}\right\rangle$ (top), degree of polarization $\left\langle\phi_{e}\right\rangle$ (center) and degree of polymerization $\left\langle\phi_{p}\right\rangle$ (bottom) as a function of self-propulsion speed $v_{0}$ for different magnetic moments $\mu$. System density is set to $\Phi=0.57$. Cut-off for cluster formation set at $\epsilon=1.05$

We verified phase separation by calculating the distribution of the local densities via Voronoi tessellation [106, 107, 131] as introduced in chapter 2. Fig. 5.12 shows 


\section{Collective dynamics in large systems without external field}

one example of a Voronoi diagram in a system with apolar particles at high selfpropulsion speed $v_{0}=61$. Here, the high density regions are colored in blue, while the coexisting low density regions are colored in red. The distribution of the densities follows a bimodal curve (black). It has been reported that dipolar interactions suppress MIPS [73] which seems to contradict claims that MIPS is promoted by velocity alignment [132]. However, dipolar interactions do not only introduce alignment but also promote positional order in form of chain formation. Chain formation could suppress MIPS, as assembly into chains reduces the number of free monomers in the dilute phase and has been speculated to suppress liquidgas coexistence [48]. We can confirm that MIPS does not emerge in systems with strong magnetic interactions. Here, the dilute phase does no longer coexist with the dense one. A more quantitative analysis regarding the coexisting densities during MIPS in systems of dipolar active particles has been performed in 73. Here, coexistence of dilute and dense regions were reported to disappear at $\mu>1$. This value corresponds to the critical magnetic strength $\mu^{*}\left(v_{0}\right)$ needed for cluster and hence chain formation of active dipolar particles, that we determined in chapter 4 A more detailed study of active dipolar systems around $\mu^{*}\left(v_{0}\right)$ could give further insights into how dipolar interactions suppress MIPS.

Calculation of global polarization (Figure 5.11) reveals that apolar particles (black curve) show no global order while systems with magnetic interactions (blue, green and red curves) exhibit high global orientational order. These observations are in agreement with $[73$. When comparing the degree of global polarization between systems with different magnetic moments $\mu$ we noticed that systems with weak magnetic interactions ( $\mu=1$, blue curve) reach higher values of polarization for lower self-propulsion speeds compared to systems with strong magnetic inter- 


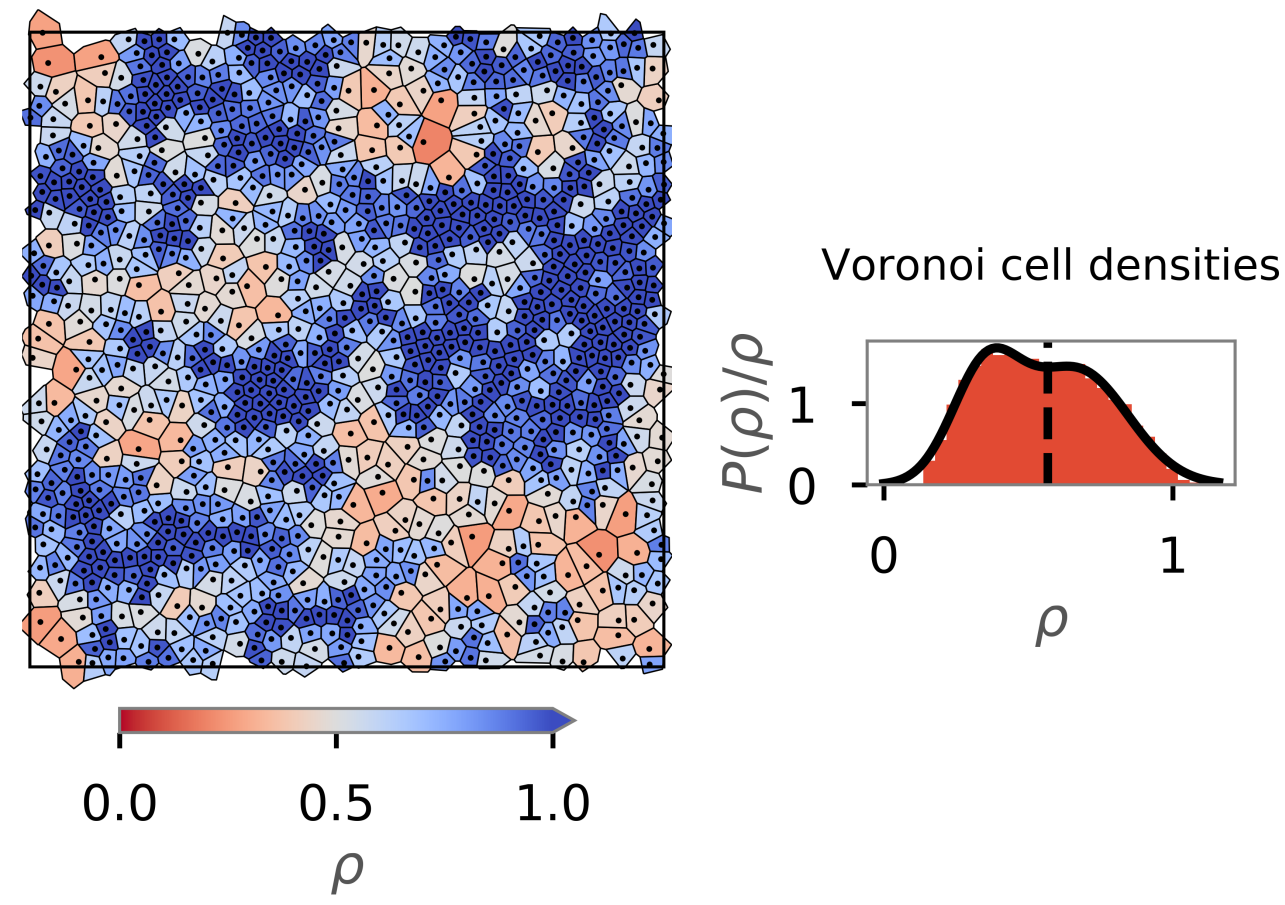

Figure 5.12.: Left: system snapshot at $t=20$ showing particle positions (black dots) and the calculated Voronoi cells. System parameters set to $\mu=0, v_{0}=$ $61, \Phi=0.57$. The cells are colored according to their density $\rho$. Right: histogram shows distribution of Voronoi cell densities $P(\rho)$ weighted with its inverse density. Black curve shows fit to bimodal distribution of cell densities, while the vertical dashed line marks the average system density $\Phi=0.57$. Histogram data was averaged over the last 5 frames.

actions ( $\mu=2.6$, red curve). In systems at low densities, we observed the opposite effect (compare Figure 5.3). To this point it remains unclear why we observed such a difference.

Figure 5.11 shows the average degree of polymerization in systems with high average density. In the apolar case $(\mu=0$, black curve) we see a strong increase of the degree of polymerization with self-propulsion. Here, values close to $\left\langle\phi_{p}\right\rangle \approx 0.5$ are being reached. Systems with $\mu \neq 0$ show high degrees of polymerization. The degree of polymerization seems to converge to approximately $\left\langle\phi_{p}\right\rangle \approx 0.9$ 
5. Collective dynamics in large systems without external field

for high self-propulsion speeds, independent of the magnetic interaction strength $(\mu \neq 0)$. Both effects together, the quick increase to high values of the degree of polymerization for apolar particles $\left(\left\langle\phi_{p}\right\rangle \approx 0.5\right)$, and unspecificity of the order parameter for systems with intermediate and strong magnetic interactions $\left(\left\langle\phi_{p}\right\rangle \approx\right.$ 1 ), suggest that the degree of polymerization is not ideally suited to characterize chain formation or polymerization in systems at high average densities.

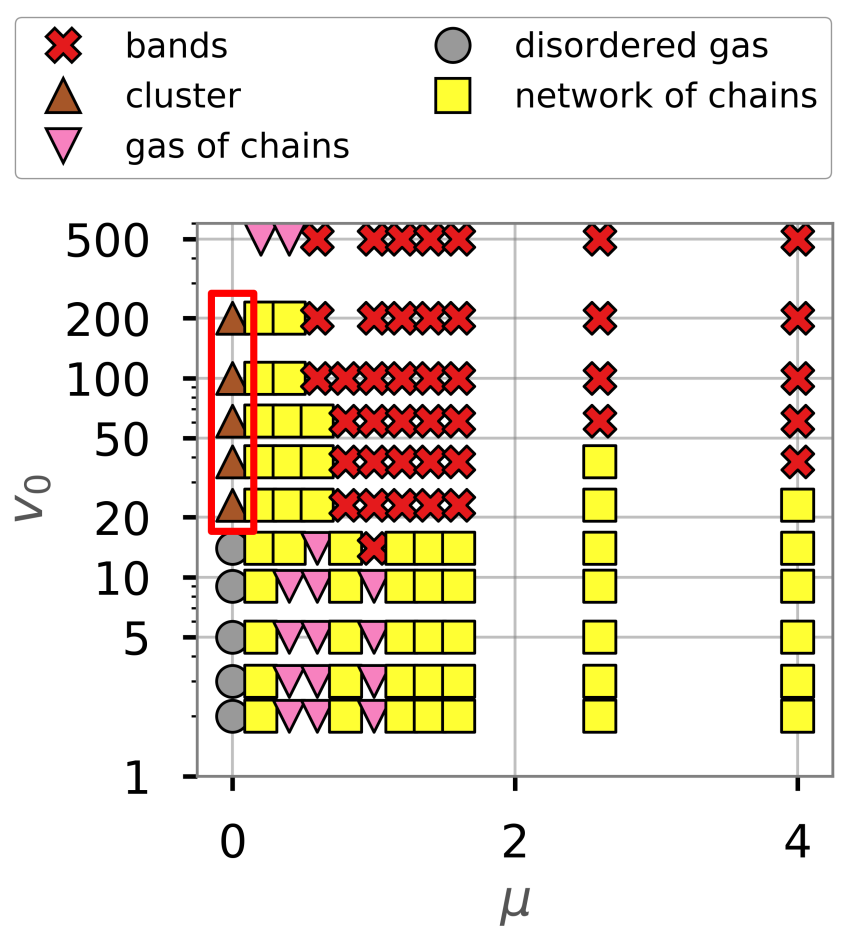

Figure 5.13.: Diagram of states for systems at high density $(\Phi=0.57)$ for different magnetic moments $\mu$ and self-propulsion speeds $v_{0}$. Red box highlights state points with MIPS as identified via Voronoi tessellation. Classification of states is based on combinations of order parameter criteria (see Table 2.1).

Again, we summarized the results of the three order parameters in a diagram of states for $\Phi=0.57$ in Figure 5.13. As already observed in systems at intermediate density (Figure 5.9), the disordered gas state (gray circle) is clearly suppressed. In addition, the oriented gas states (purple plus) vanish from the diagram of states. 
Instead, purely clustered states (brown triangle, highlighted with red box) emerge for apolar particles at high self-propulsion speeds. These states were identified as MIPS and is qualitatively in agreement with $[73]$. Overall, complex networks of chains (yellow square) and bands (red cross) dominate the diagram of states at high density.

Now that we have identified and characterized in detail how activity, magnetic interactions and density affect the collective behavior of systems of active dipolar particles, we study the influence of a constant external magnetic field in a next step. 


\section{Influence of constant external magnetic field}

In this chapter, we ask the question how does a constant homogeneous external magnetic field affect the collective dynamics in a large system of dipolar particles in bulk? First, we present simulation snapshots of typical configurations we observed. Then we discuss how the complex interplay between external magnetic field and internal dipole-dipole interactions between the particles affects structure formation of active dipolar particles. We classified observed states in our system according to three order parameters and summarized our results in diagrams of states.

To this extent, we analyzed the results of numerical simulations of $N=1156$ active dipolar particles for three different densities (low density $\Phi=0.13$, intermediate density $\Phi=0.23$ and high density $\Phi=0.57$ ) with periodic boundary conditions for 7 different values of magnetic field strengths in the interval $B=$ $10^{-2}$ to $10^{2}$. We varied magnetic strength $\mu$ and self-propulsion speed $v_{0}$ of the particles. 


\subsection{Low density $\Phi=0.13$}

Figure 6.1 shows typical system configurations for three different values of the magnetic moments $\mu=1.0,1.4$ and 2.6 (columns) at three different field strengths of the external magnetic field $B=0.1,1.0$ and 14 (rows) for particles with moderate self-propulsion speed $\left(v_{0}=23\right)$.
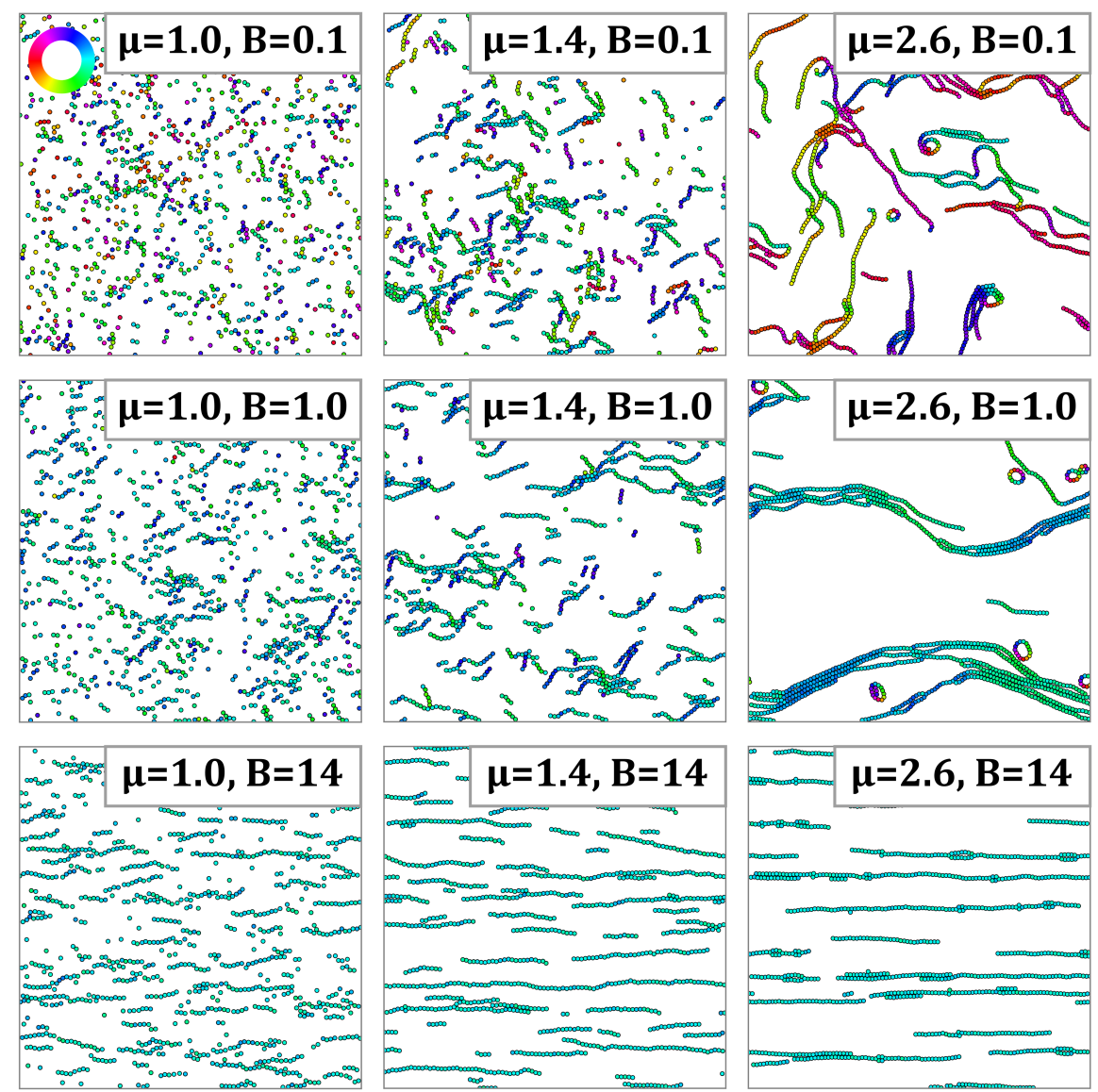

Figure 6.1.: Collection of snapshots of the system at low density $(\Phi=0.13)$ for intermediate self-propulsion speed $v_{0}=23$ for different magnetic field strengths $B$ (rows) and values for magnetic interaction strengths $\mu$ (columns). External magnetic field points from left to right. All snapshots have been taken at time $t=20$.

Systems with weak external magnetic field display similar configurations to what 


\section{Influence of constant external magnetic field}

we observed in systems without external magnetic field (chapter 5). In systems with stronger external magnetic fields, particles align with the direction of the external field (left to right, cyan). In addition, particles assemble into chains with defects like short branches or loops for strong external magnetic fields $(B=14)$. Here, the average chain length seems to increase with the magnetic moment of the particles, spanning the whole system size for strong magnetic interactions and strong external field strengths $(\mu=2.6, B=14$, bottom right). These columnar structures are qualitatively similar to structures observed in non-equilibrium ferrofluids with external magnetic field [57]. In contrast to systems without an external magnetic field, these columnar structures mostly stay evenly separated and do not combine into connected networks of chains or into broad bands at low densities. In fact, we observed in systems with strong magnetic interactions ( $\mu=2.6)$ that an increase of the external magnetic field strength causes broad bands to separate into bands with fewer lanes or into individual chains of dipolar particles (e.g. Figure 6.1 $\mu=2.6, B=1.0$ and $\mu=2.6, B=14$ ).

We analyzed the numerical simulations quantitatively by calculating three global order parameters, as introduced in chapter 2, In general, the observations above are also reflected in the calculated order parameters, which are shown in Figure 6.2. In particular, we have seen that the fraction of largest cluster $\left\langle\phi_{c}\right\rangle$ (top) decreases with increasing magnetic field strength for high magnetic moments $\mu$ (red lines) while the degree of polymerization $\left\langle\phi_{p}\right\rangle$ (bottom) slightly increases with increasing field strength. Furthermore, $\left\langle\phi_{c}\right\rangle$ and $\left\langle\phi_{e}\right\rangle$ weakly depend on self-propulsion speed $v_{0}$ in systems with strong external magnetic fields.

Naturally, we observed a strong increase of the degree of polarization $\left\langle\phi_{e}\right\rangle$ (center) with increasing field strength. The average alignment of a single magnetic 


$\longrightarrow \mu=0.0-\mu=1.0 \quad \mu=1.4 \quad \mu=2.6$
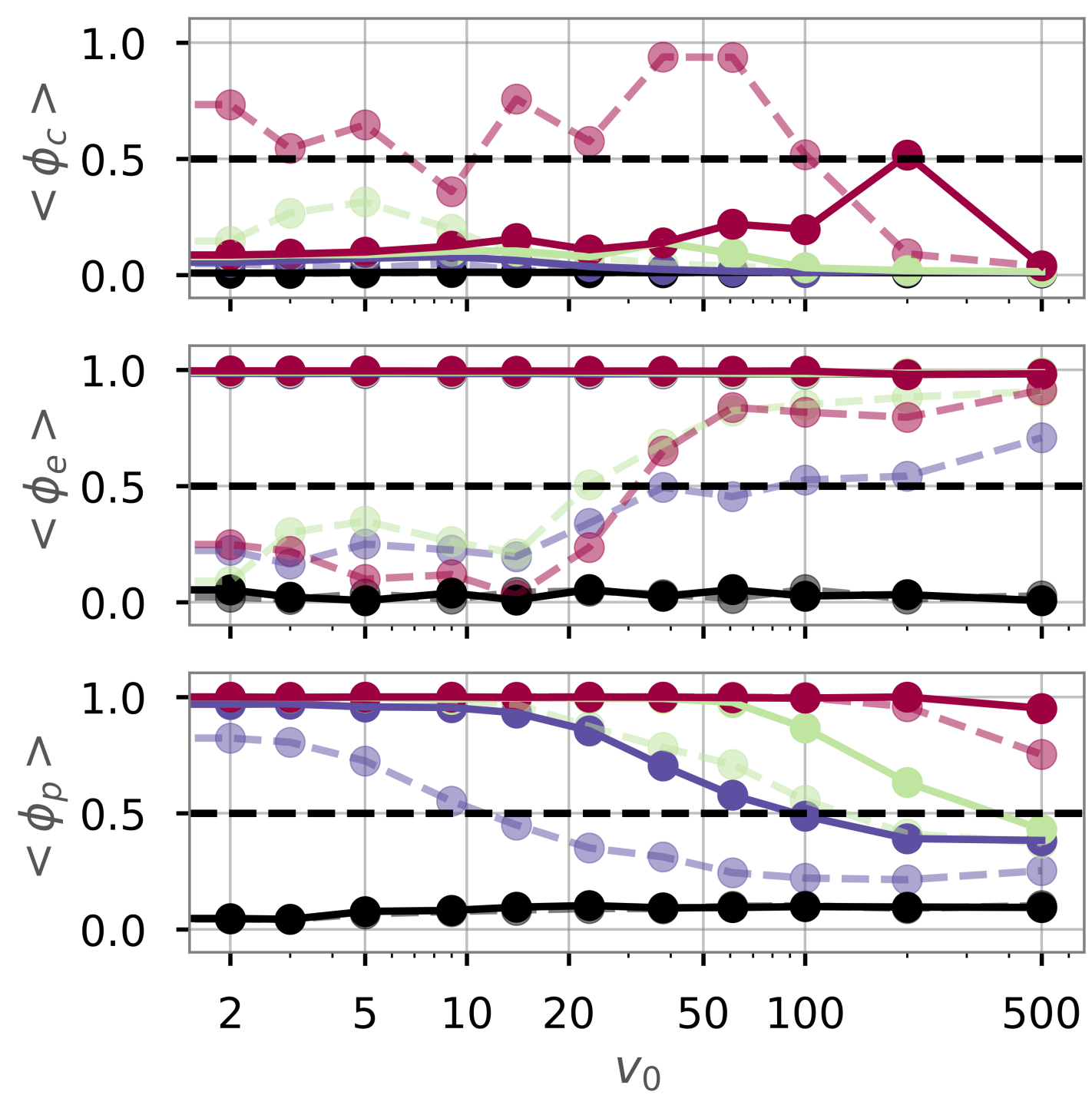

Figure 6.2.: Three order parameters: fraction of largest cluster (top), polarization (center) and polymerization (bottom) against self-propulsion speed $v_{0}$ for four magnetic moments $\mu$ (colors). Solid color and solid lines show data for strong external magnetic field $B=14$, transparent colors and dashed lines show data with weak magnetic field strength $B=0.1$. System density was set to $\Phi=0.13$. Cut-off for cluster formation set at $\epsilon=1.28$. 


\section{Influence of constant external magnetic field}

dipole with strength $\mu$ in an external magnetic field of strength $B$ in a thermal bath at temperature $T$ is described by the Langevin function (in reduced units) 133

$$
\left\langle\phi_{e}\right\rangle^{B}=\operatorname{coth} \frac{\mu B}{T}-\frac{T}{\mu B}
$$

In Figure 6.3 we compared the expected orientation of the particles according to the Langevin function (black line) to the measured average orientation in our system $\left\langle\phi_{e}\right\rangle$ (colored dots). Because of particle-particle interactions and alignment with neighboring particles, most of our data points lie above the Langevin curve. However, we noticed some outliers well below the expected average orientation. A snapshot of one of these outliers (red cross) reveals why. In systems with weak external magnetic fields but strong magnetic moments of the particles, rings or more complex circular structures can form which reduce the average orientation of the system.

Additionally, based on the expected average orientation of a reference system $(\mu=1, T=1)$, we defined three magnetic field strength regimes: low $(B=0.1)$ intermediate $(B=1)$ and strong $(B=14)$, as indicated by the vertical dashed lines. We present our simulation data representatively for these three regimes.

We summarized the results of the order parameters in a diagram of states following the classification criteria of Table 2.1 in Figure 6.4. Here, diagrams of states for three different magnetic field strengths $B=0.1,1.0$ and 14 (left to right) for density $\Phi=0.13$ are shown. We can identify three crucial observations.

First, disordered gas states (gray circle) are greatly suppressed with increasing magnetic field strength. Additionally, the onset of oriented gas states states shifts to lower values of $\mu$. 

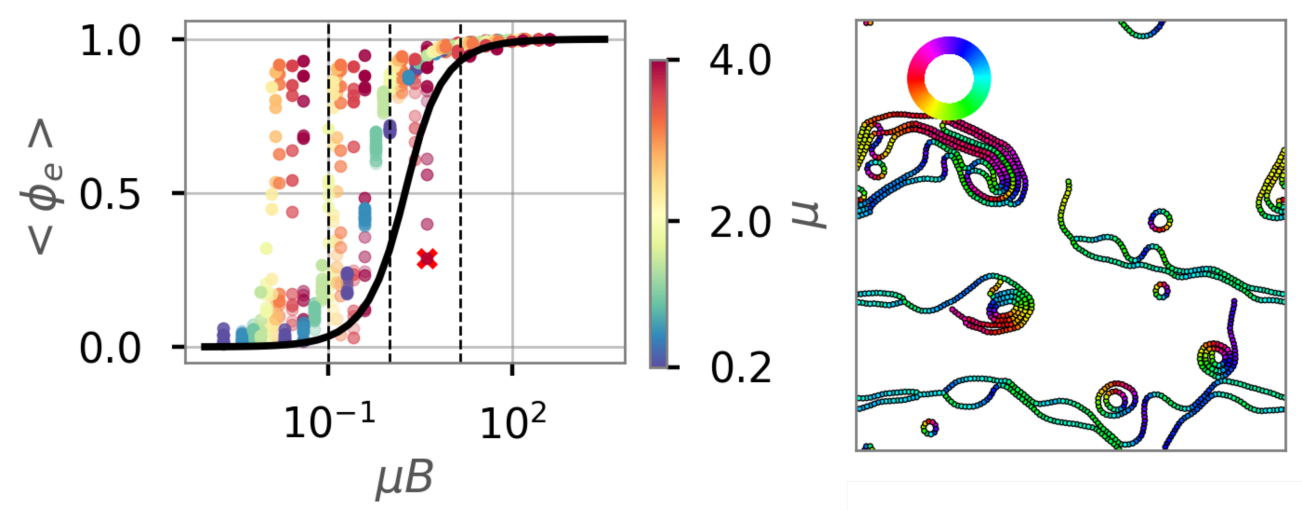

Figure 6.3.: Left: expected orientation of single particle in external magnetic field based on Langevin equation (6.1) (black line) with $T=1$ and the measured average orientation in our system for different magnetic moments $\mu$ color coded and self-propulsion speeds $v_{0}$ (indicated by transparency of dots, higher values of $v_{0}$ show up less transparent). Vertical lines indicate three magnetic field strength regimes for reference system wit $\mu=1$ : low $(B=0.1)$ intermediate $(B=1)$ and strong $(B=14)$. Right: snapshot of system at parameters $\mu=4, B=1$ and $v_{0}=23$ (red cross in left figure). Particle orientations are indicated by the color wheel (top left). System at density $\Phi=0.13$. Snapshot taken at $t=20$.

Second, intermediate external magnetic fields (center diagram) suppress the formation of complex networks of chains (yellow square) and instead promote the formation of oriented states (purple plus: oriented gas, green pentagon: oriented chains) and bands (center, bands: red cross).

Third, and most crucially, strong external magnetic fields suppress the formation of bands. For strong external magnetic fields (right diagram), two states dominate the diagram: oriented gas (purple plus) and oriented chains (green pentagon). The line separating these two states is qualitatively similar to the line separating the gas and chain state in small systems of dipolar active particles (see Figure 4.10 , chapter 4).

We want to highlight the two states indicated as bands (red cross) for strong external magnetic field in Figure 6.4 (right). At these two parameters $(\mu=2.6$, 


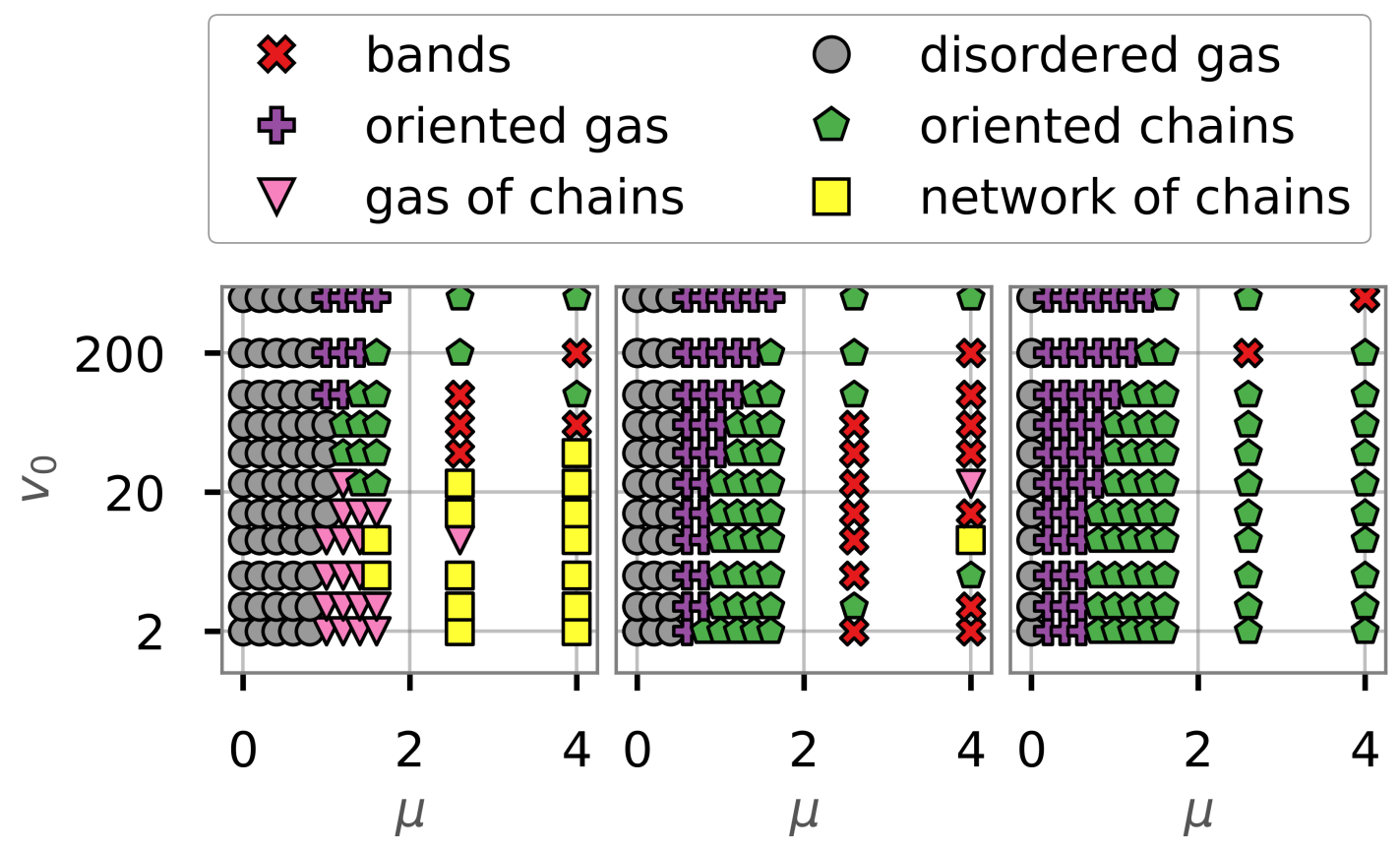

Figure 6.4.: Diagrams of states for three different external magnetic field strengths $B=0.1,1.0$ and 14 (left to right). Classification of states is based on combinations of order parameter criteria (see Table 2.1). System at low density $(\Phi=0.13)$.

$v_{0}=200$ and $\left.\mu=4, v_{0}=500\right)$ we observed the following behavior, as shown in Figure 6.5. Dipolar active particles that self-assembled into long chains oscillate with growing amplitude towards their tail. We suspect that such an oscillation might be caused by a buckling instability. Particles with strong dipolar interactions are packed closely into a chain. The combination of high swimming speeds and translational noise might cause a temporary compression and stress along the chain that is then resolved by buckling of the tail. Self-assembled active dipolar chains are qualitatively similar to active polymers, where monomers are linked by a spring potential. Here, it has been shown that the flexure number, which characterizes the relation between active propulsion and bending rigidity, controls 
buckling instabilities in a single active filament [134]. To our knowledge, oscillating chains have not been observed before in systems of active dipolar particles. Further studies are required to explore this behavior.

When dipolar interactions were reduced, long chains were seen to break into smaller segments. These breaks typically occurred near the tail of the chain, where the oscillations reach their maximum amplitude. This observation indicates that buckling instabilities might cause chains of different lengths to coexist in systems of active dipolar particles. Breaking of chains is qualitatively similar to fragmentation of stationary chains observed in magnetorheological suspensions in a rotating field 66,135 .

So far, we have observed that these oscillating chains are a transient state towards band formation in our systems. At some point, individual oscillating chains meet. Here, alignment with neighboring particles suppresses oscillations and bands start to form. Similar lateral aggregation of individual chains into bands and columnar lanes has been observed in magnetorheological fluids [57, 62, 63, 65].

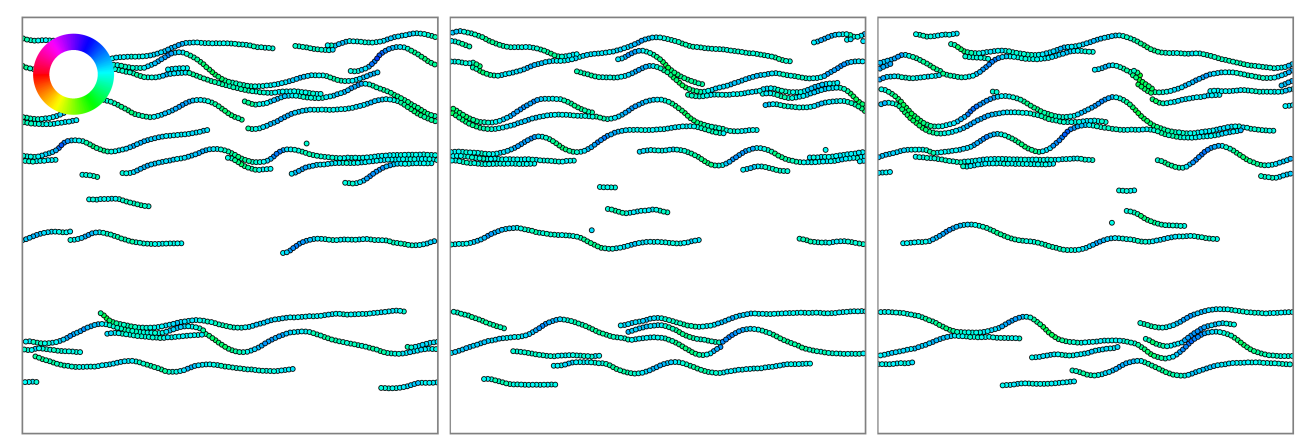

Figure 6.5.: Series of snapshots showing oscillating chains for dipolar active particles with strong magnetic interactions $\mu=4$ and high self-propulsion speed $v_{0}=500$. Snapshots taken at times 26.46, 26.56 and 26.66 (left to right), color wheel indicates orientations. External magnetic field points from left to right, magnetic field strength was set to $B=14$. 


\section{Influence of constant external magnetic field}

In addition, we also investigated the effect density has on systems of active dipolar particles in external magnetic fields. To this extent, we repeated the calculation of the order parameters above for systems with densities $\Phi=0.23$ and $\Phi=0.57$. We summarized our results in diagrams of states.

\subsection{Intermediate density $\Phi=0.23$}

In systems at intermediate density $\Phi=0.23$, we observed that intermediate external magnetic field strengths of $B=1$ do not suffice to suppress formation of complex networks. Because of the increase in density, short chains may connect and form a network (see Figure 6.6 central column, $\mu=1.4$ ).

This observation is also reflected in the diagram of states (Figure 6.7, yellow squares). Furthermore, the diagram of states indicates that strong external magnetic fields diminish the effect activity has in systems at intermediate density (Figure 6.7, right). Here, the line separating the two states oriented gas (purple plus) and oriented chains (green pentagon) is independent of $v_{0}$. This observation is dramatically different to the previously characterized systems without external magnetic fields and to systems at low density with external magnetic fields. Here, we have demonstrated that activity greatly affects structure formation and the collective behavior.

In the diagram of states we demonstrated that band formation (red cross) is suppressed for strong external magnetic fields in systems at low (Figure 6.4) and intermediate (Figure 6.7) densities. Instead, oriented chains dominate. This effect seems to be more dominant at intermediate density $\Phi=0.23$. Therefore, we will focus on systems at intermediate density and study how external magnetic fields 


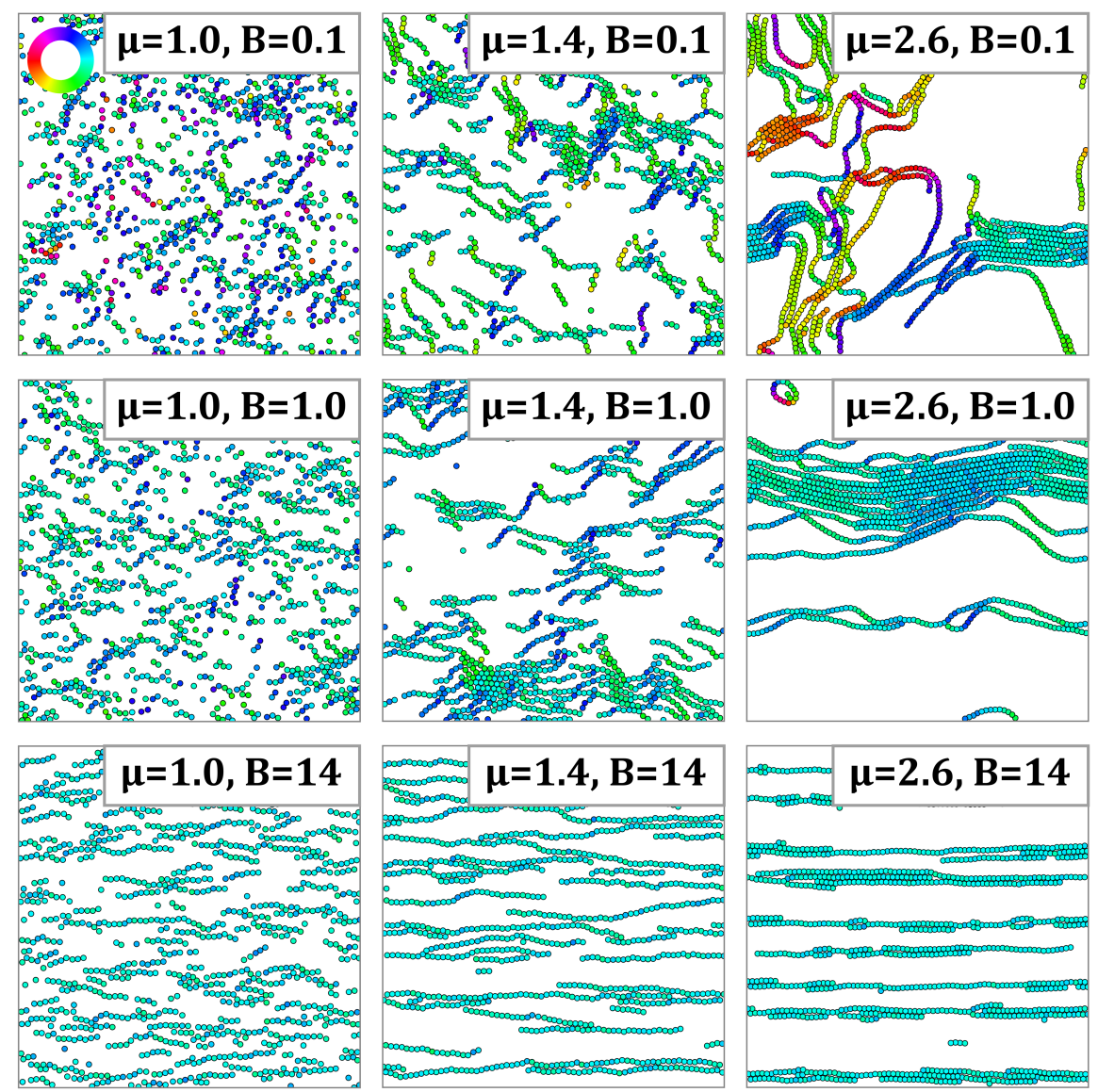

Figure 6.6.: Collection of snapshots of the system at intermediate density $(\Phi=$ $0.23)$ for intermediate self-propulsion speed $v_{0}=23$ for different magnetic field strengths $B$ (rows) and magnetic moments $\mu$ (columns).

affect lane formation in more detail.

\subsubsection{Columnar structures}

The snapshots in Figure 6.6 show that as the magnetic field strength increases, the disordered network of chains first organizes into a broad band. Then, as the field strength increases further, the band separates into columnar structures. These structures are well aligned with the direction of the external field, spanning the 


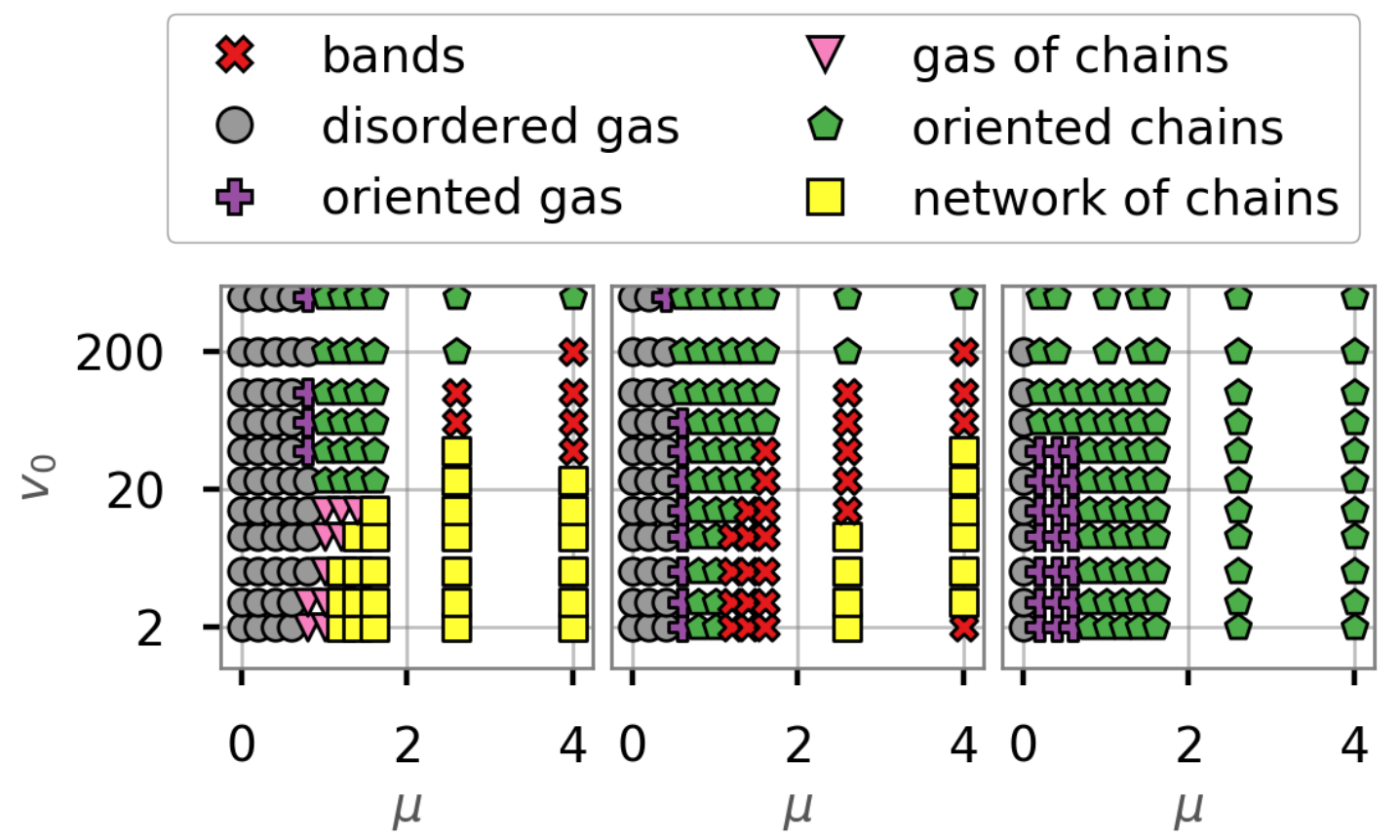

Figure 6.7.: Diagrams of states for three different external magnetic field strengths $B=0.1,1.0$ and 14 (left to right). Classification of states is based on combinations of order parameter criteria (see Table 2.1). System at intermediate density $(\Phi=0.23)$. Cut-off for cluster formation set at $\epsilon=1.21$.

system for strong magnetic interactions. Snapshots of the simulations suggest (compare Figure 6.1, Figure 6.6 and Figure 6.10), that the number of lanes in each columnar structure can vary and is greatly influenced by the density. In addition, these columnar structures seem to be quite regularly spaced in the direction perpendicular to the external magnetic field. These observations qualitatively match magnetic-field-induced structures in ferrofluids $[57,62,63]$.

We characterized these columnar structures with two parameters: the average number of lanes in a columnar cluster $\overline{n_{1}}$ and the columnar spacing $\overline{\Delta r_{y}}$, which is the average distance between neighboring cluster centers perpendicular to the external magnetic field. 

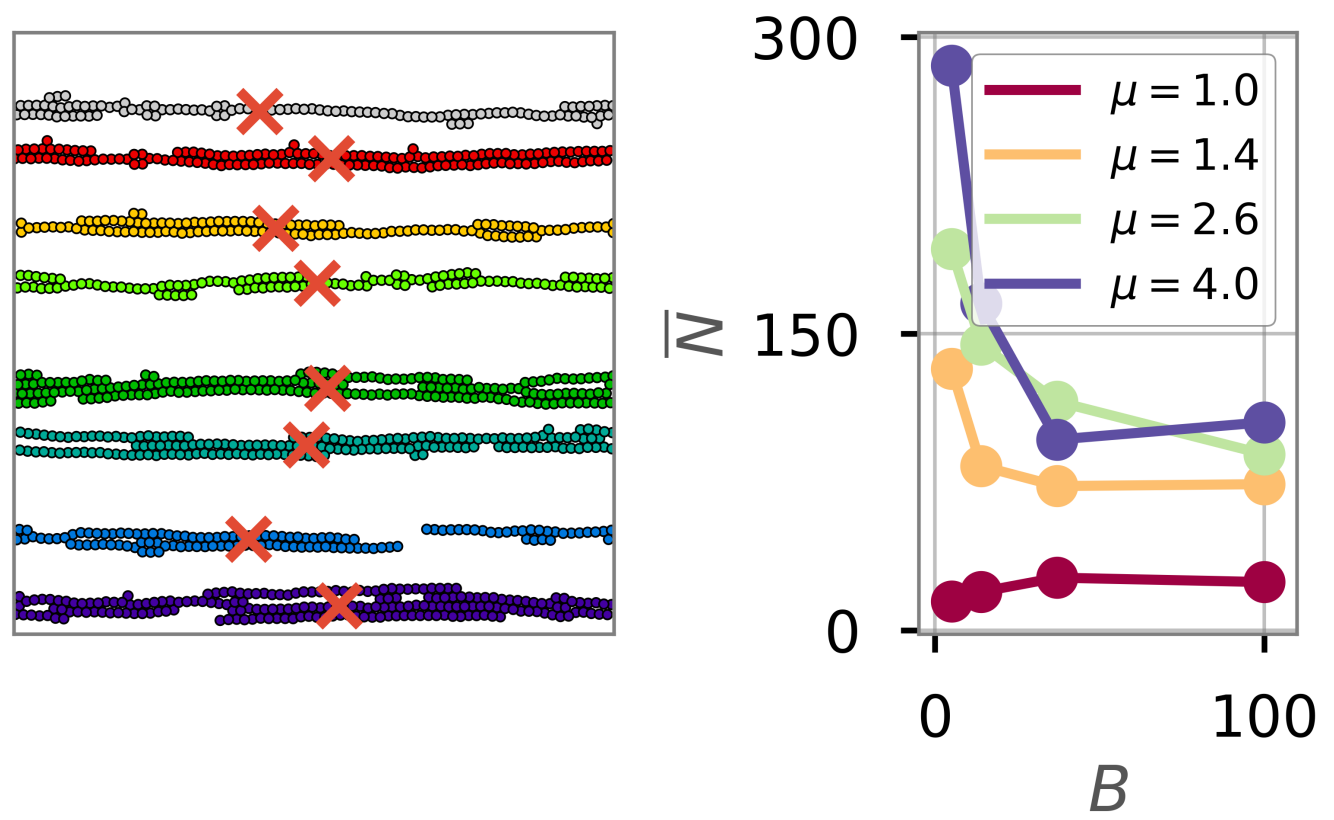

Figure 6.8.: Left: snapshot of system at $B=14, \mu=2.6, v_{0}=14$ showing columnar structures. Structures are color coded, center of geometry is marked by a red cross. Right: average cluster size $\bar{N}$ against external magnetic field strength $B$ for particles with moderate self-propulsion speed $v_{0}=14$. System is at intermediate density $\Phi=0.23$. Colors indicate different magnetic moments of the dipolar particles. Data sampled over trajectories at times $t=18-20$ every $\Delta t=0.1$.

These two parameters were calculated as follows. First, we determined the individual clusters (color coded in Figure 6.8, left), using a density based cluster algorithm (see chapter 2). We then calculated the center of geometry (red cross) and the length of each cluster in direction of the external magnetic field $l_{x}^{i}$. Averaging the distances between neighboring centers of geometry perpendicular to the direction of the external magnetic field gives us the columnar spacing. To approximate the number of lanes in each cluster, we divided the number of particles per cluster $n_{i}$ by the cluster length

$$
n_{1}^{i}=\frac{n_{i}}{l_{x}^{i}}
$$




\section{Influence of constant external magnetic field}

We determined these parameters for systems that fulfill the criteria for polarization $\left(\left\langle\phi_{e}\right\rangle>0.5\right)$ and polymerization $\left(\left\langle\phi_{p}\right\rangle>0.5\right)$, corresponding to systems in oriented chains or bands states (see Table 2.1).

Indeed, the calculation of the average number of particles per cluster $\bar{N}$ (Figure 6.8, right) shows a strong decrease in average cluster size with increasing magnetic field strength, confirming that clusters separate into smaller sized clusters as the magnetic field strength increases in systems with $\mu>1$. Here, the average cluster size seems to converge to a fixed value for very strong external magnetic fields.
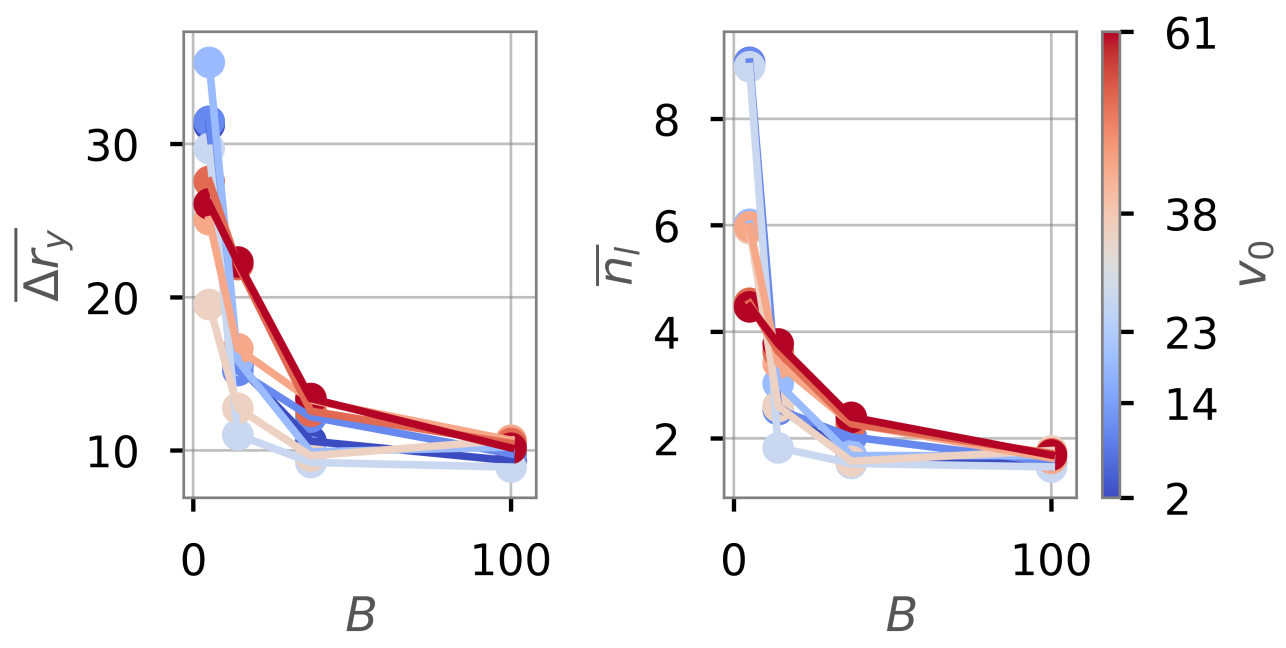

Figure 6.9.: Columnar spacing $\overline{\Delta r_{y}}$ (left) and average number of lanes per cluster $\overline{n_{1}}$ (right) against external magnetic field strength $B$. The magnetic moment was set to $\mu=4$ and density to $\Phi=0.23$. Colors indicate increasing self-propulsion speed of the particles. Data sampled over trajectories at times $t=18-20$ every $\Delta t=0.1$. Data points show systems that fulfill criteria $\left\langle\phi_{e}\right\rangle>0.5$ and $\left\langle\phi_{p}\right\rangle>0.5$.

Fig. 6.9 shows the columnar spacing $\overline{\Delta r_{y}}$ and the average number of lanes per column $\overline{n_{1}}$ for systems with strong magnetic interactions $\mu=4$ at intermediate density $\Phi=0.23$. Both, the columnar spacing and the number of lanes per cluster 
decay rapidly and approach a fixed value as the external magnetic field strength increases. Activity seems to affect this behavior only to little extent.

Columnar spacing has been reported to be independent of the external magnetic field in experiments with ferrofluids $[63]$. We recovered this behavior for strong external magnetic fields in our systems of active particles. However, we observed a strong dependence of the columnar spacing in the regime of weak external magnetic fields. We note that in some cases, a weak external magnetic field seems to promote separation between columnar structures.

As reported in [63], columnar spacing is reduced with increasing density. Although we observed in movies of our simulations that the number of lanes in each column increases with density, we cannot make quantitative statements about the influence of density on columnar structures at this point.

\subsection{High density $\Phi=0.57$}

Finally, we studied the effect of a constant external magnetic field in systems at high density $\Phi=0.57$. Here, we observed that strong external magnetic field strengths of $B=14$ clearly organize the system with high magnetic moments into columnar structures (see Figure $6.10 \mu=2.6, B=14$ ). Because of the increase in density, these columnar structures are made up of multiple lanes in off-register alignment and not of single lanes, as seen in systems at low density.

In the diagram of states, we observed that the increased density causes the previously suppressed band states (red crosses) to reappear. In fact, bands dominate the corresponding diagram of states for strong external magnetic fields and are independent of activity (Figure 6.11, right). Off-register band formation has been 
6. Influence of constant external magnetic field
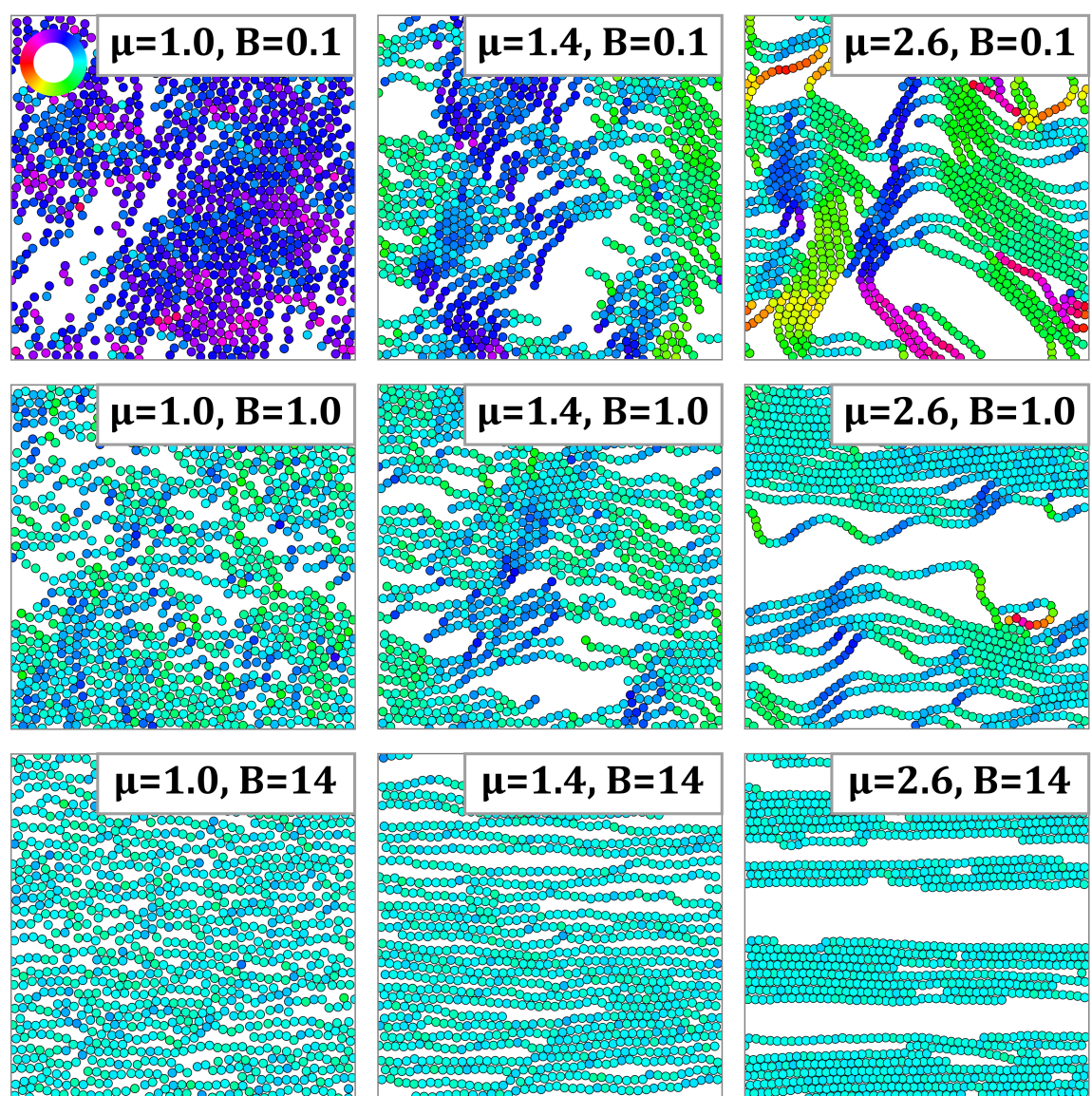

Figure 6.10.: Collection of snapshots of the system at high density $(\Phi=0.57)$ for intermediate self-propulsion speed $v_{0}=23$ for different magnetic field strengths $B$ (rows) and magnetic moments $\mu$ (columns).

previously observed in passive dipolar systems with constant external magnetic fields [39, 129, 130]. In systems at high density, a clear discrimination between bands and columnar states can no longer be made. A limiting factor might be the finite system size, as observed in other systems of active particles 136. 
6.3. High density $\Phi=0.57$

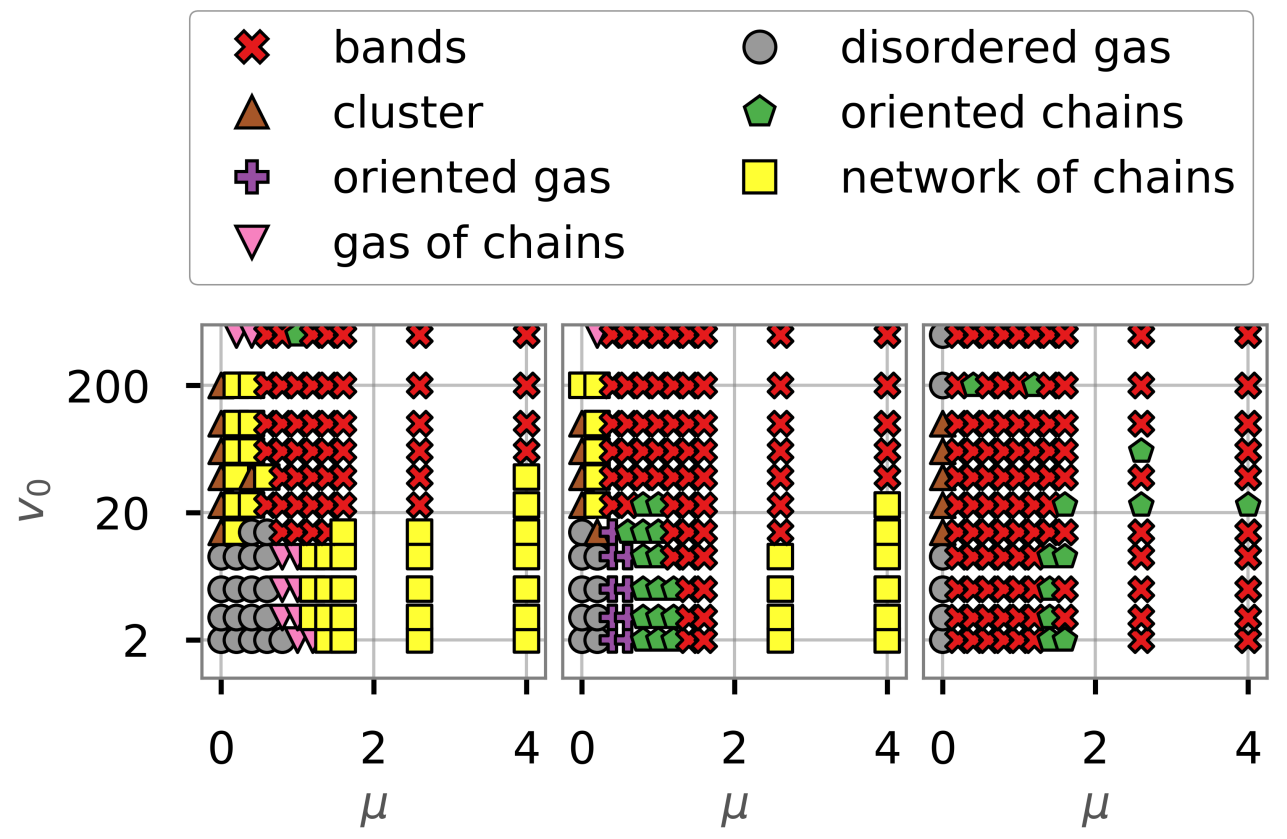

Figure 6.11.: Diagrams of states for three different external magnetic field strengths $B=0.1,1.0$ and 14 (left to right). Classification of states is based on combinations of order parameter criteria (see Table 2.1). System at high density $(\Phi=0.57)$. Cut-off for cluster formation set at $\epsilon=1.05$. 


\section{Collective dynamics in time-dependent external field}

In the previous chapter, we have demonstrated how a constant external magnetic field affects self-assembly of structures of active dipolar particles. In this chapter we investigate the effect of a time dependent external magnetic field. We model the time dependency of the external magnetic field as a triangular wave with amplitude $B$ and ramping rate $\beta$, as introduced in chapter 2 .

In particular, we are interested how the ramping rate, magnetic interaction strength and self-propulsion speed of the particles affect structure formation and previously characterized states. First, we demonstrate how the average orientation of the system changes with the time dependent external magnetic field. Then, motivated by movies of our simulations, we characterize the dynamic structure formation that is introduced with the time dependent external magnetic field.

To that end, we investigate numerical simulations for four different ramping rates $\beta=0.4,0.8,1.2$ and 4.0 while varying the magnetic moments $\mu$ and selfpropulsion speed $v_{0}$ of the particles at three different densities $\Phi=0.13,0.23$ and 0.57 . 


\subsection{Simulation observations}

\subsection{Simulation observations}

Dipolar particles align with the direction of the external magnetic field. When we apply our time-dependent external magnetic field with triangular modulation, we observed that the average orientation of the system $\phi_{e}$ follows a hysteresis curve when plotted as a function of the magnetic field strength $B$, as shown in Figure 7.1 Here, negative values of the magnetic field strength indicate a change in direction (right to left). Positive (negative) values for the average orientation $\phi_{e}$ at positive (negative) magnetic field strength $B$ signify alignment with the direction of the external magnetic field, when pointing from left to right (right to left). Particles are fully aligned when the external magnetic field reaches its maximum amplitude. During the course of one simulation run particles switch their swimming direction twice, which results in a closed hysteresis loop.

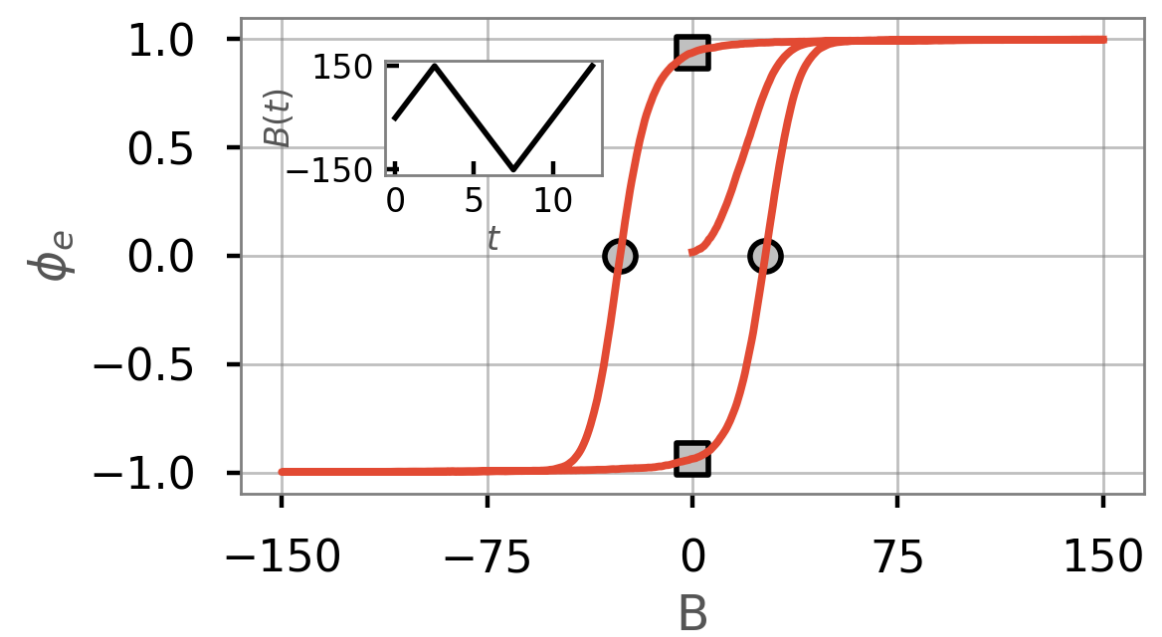

Figure 7.1.: Hysteresis curve for system with parameters $\mu=0.5, v_{0}=100$, $\beta=0.4$ and $\Phi=0.13$. Inset shows time dependency of external magnetic field with amplitude $B=150$. Gray circles mark the coercive force and gray squares mark the normalized remnant magnetization. Points were calculated from intersection via linear interpolation. 


\section{Collective dynamics in time-dependent external field}

In movies of our simulations, we typically observed the following behavior (Figure 7.2. As the external magnetic field strength ramps up, particles combine into chains and align with the direction of the external magnetic field (Figure 7.2 , left). The particles move along the external magnetic field from left to right (cyan). The length of the chains depends on the magnetic moment of the particles. Particles with small magnetic moments $(\mu=0.5)$ do not combine into chains but stay separated. As the external magnetic field strength decreases, particles start to lose their alignment with the external field and eventually reorient (Figure 7.2 , center), as the external magnetic field switches its direction (right to left). During reorientation, chains may break into smaller segments or individual particles. Finally, as the external magnetic field strength deceases further, particles recombine into long chains which are aligned with the new direction of the external magnet field. Now particles move from right to left (red) (Figure 7.2, right).

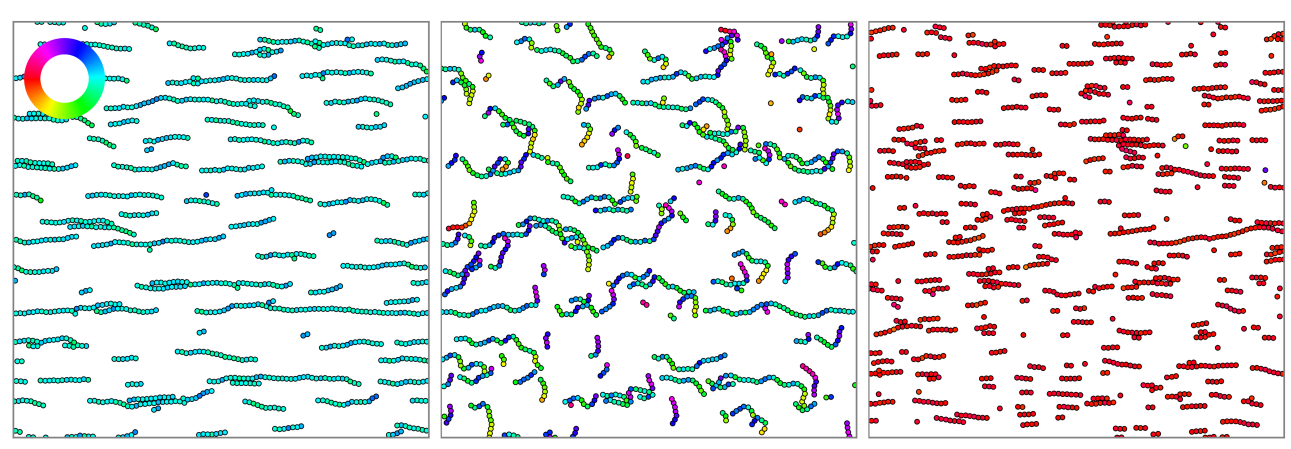

Figure 7.2.: Time series of snapshots for system with $\beta=0.4, \mu=2, v_{0}=40$ and $\Phi=0.13$. Snapshots are taken at times $t=10.53,11.03$ and 11.53 (left to right). Color wheel (top left) indicates orientation of particles. External magnetic field points from left to right (right to left) at time 10.53 (11.53). 
7.2. Coercive force and relative remnant magnetization

\subsection{Coercive force and relative remnant}

\section{magnetization}

As shown in Figure 7.1, particles do not reorient when the external magnetic field strength is zero. Reorientation of the particles requires a certain field strength $B_{c}$. In this section, we want to study the exact point when the particles reorient and how it depends on the ramping rate $\beta$, magnetic moments $\mu$ and activity $v_{0}$.

We used two points to characterize hysteresis curves of our system, as indicated in Figure 7.1. The coercive force (gray circle) and the normalized remnant magnetization (gray square). The coercive force is the magnetic field strength $B_{c}$ required to demagnetize the system $\phi_{e}\left(B_{c}\right)=0$. At this point, particles are starting to reorient. The normalized remnant magnetization $\phi_{e}^{*}$ is the systems' average orientation that remains when the field strength of the external magnetic field is equal to zero $\phi_{e}^{*}=\phi_{e}(B=0)$.

We now ask, how does the coercive force $B_{c}$ and the normalized remnant magnetization $\phi_{e}^{*}$ depend on the systems' parameter? Fig. 7.3 shows the coercive force and the normalized remnant magnetization against the activity for three magnetic moments $\mu$. The semi-transparent curves with dotted lines show data for slow ramping rates $\beta=0.4$ and the solid curves with solid lines show data for higher ramping rates $\beta=1.9$. The relative remnant magnetization is overall high $\left(\phi_{e}^{*}>0.9\right.$, notice small y-range) and the observed changes with self-propulsion speed $v_{0}$ are negligibly small (Fig. 7.3 , bottom). Systems with high ramping rates and low magnetic moments (blue solid curve) require higher values of the external magnetic field strength for particles to reorient. This observation is to be expected since the degree of alignment in an external field of a dipolar particle follows a 
7. Collective dynamics in time-dependent external field

Langevin curve (see chapter 2). Both parameters show little to no dependency on activity and system density (not shown). In systems with constant external magnetic field, we saw that activity and density affected the collective motion of dipolar particles (chapter 6). Typically, magnetization curves of ferromagnetic materials depend on the temperature $68,137,138$. This observation once more shows the limits of interpreting activity as an effective temperature [120].

In systems with a time dependent external magnetic field, the dynamics were not seen to be affected by the activity of the particles. Here, the temporal evolution of the external magnetic field dictates the dynamics of the self-assembly of active dipolar particles.

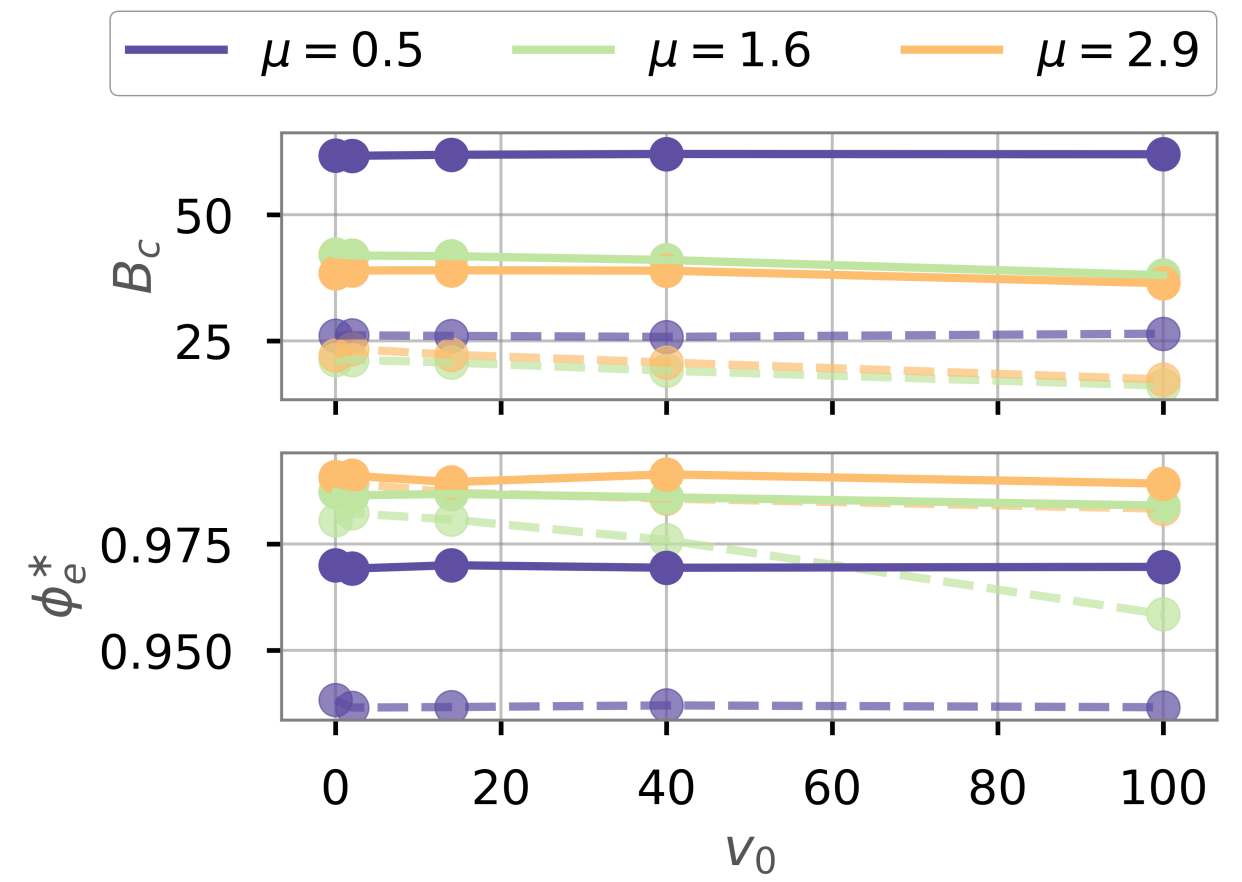

Figure 7.3.: Coercive force (top) and relative remnant magnetization of the system against self-propulsion speed $v_{0}$ for three different magnetic moments $\mu$. Semitransparent dotted curves show systems with low ramping rate $(\beta=0.4)$ while solid curves show systems with high ramping rate $(\beta=1.9)$. System density set to $\Phi=0.13$ 


\subsection{Dynamic structure formation}

\subsection{Dynamic structure formation}

One crucial feature in a time dependent external magnetic field is that particles reorient shortly after the external magnetic field switches its direction (hysteresis). In this section, we will focus on the structures that are adopted during this transition. In general, we observed three distinct scenarios in movies of our simulations. In systems with particles with small magnetic moments, particles do not assemble into chains but remain separated. Individual particles re-align with the external magnetic field by performing a U-turn. In systems with intermediate and high magnetic moments, two possible observations were made. Here, either already formed chains break into smaller segments and individual particles, reorient and recombine into new chains. Or initially formed chains stay intact and reorient as whole in a U-turn manner, temporarily adopting a C-shaped chain before realigning with the new direction of the external magnetic field (see Figure 7.2 , center).

We analyzed such dynamic behavior while varying the parameters $\beta, \mu$ and $v_{0}$. We classified these three scenarios by calculating the degree of polymerization $\phi_{p}$ in our simulations in a time frame around the time point when particles reorient. Particles reorient at time $t^{*}=t\left(\phi_{e}=0\right)$ when the average orientation is zero. The time frame was chosen to include $\Delta t=0.15$ before and after that time point.

Fig. 7.4 shows an example of this calculation for three different parameter combinations $\mu=0.5, v_{0}=40$ (gray), $\mu=1.7, v_{0}=100$ (red) and $\mu=4, v_{0}=40$ (blue). Here, the above mentioned three scenarios can be distinguished. The degree of polymerization is low $\left(\left\langle\phi_{p}\right\rangle<0.5\right)$, therefore particles do not assemble into chains (gray). The degree of polymerization is high $\left(\left\langle\phi_{p}\right\rangle>0.5\right)$, but has a 
7. Collective dynamics in time-dependent external field

distinct minimum, therefore particles assemble into chains but break when reorienting (red). The degree of polymerization is high $\left(\left\langle\phi_{p}\right\rangle>0.5\right)$, therefore particles assemble into chains that do not fragment into monomers when reorienting (blue).

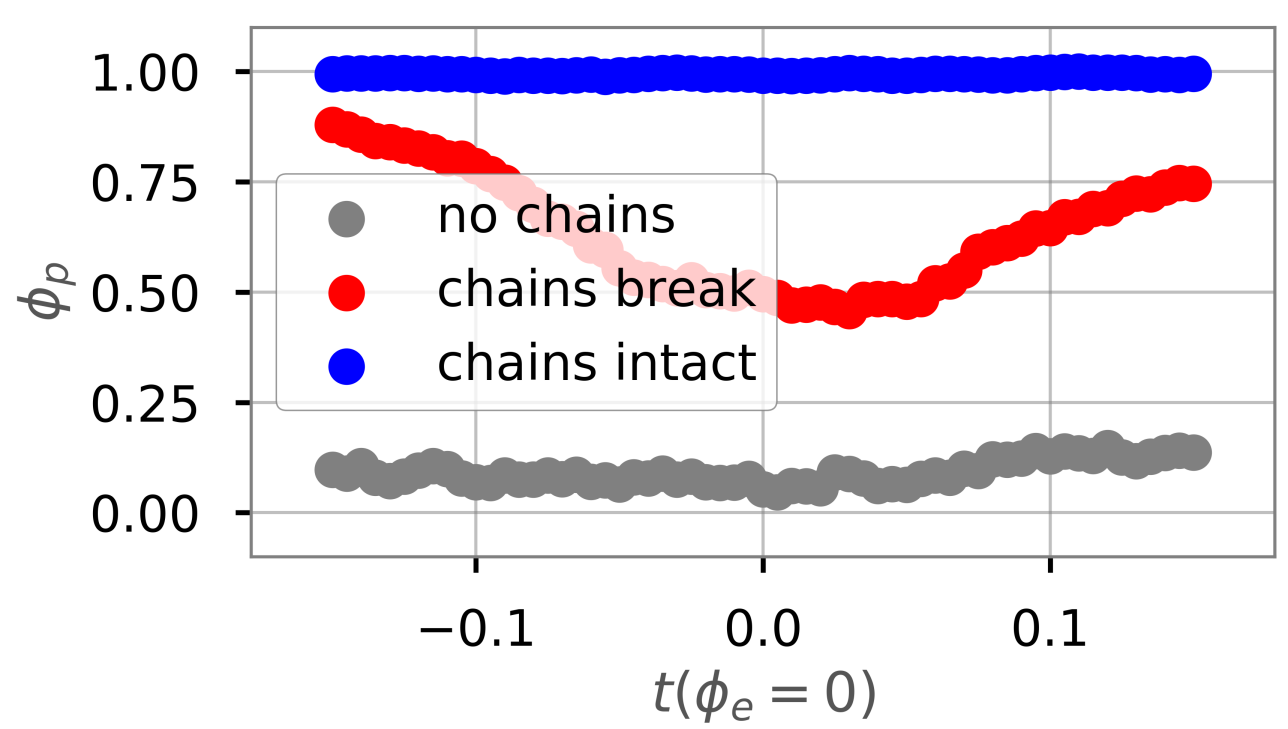

Figure 7.4.: Temporal evolution of order parameter $\phi_{p}$ around reorientation point showing three distinct scenarios for dynamic structure formation $\left(\mu=0.5, v_{0}=40\right.$ : gray, $\mu=1.7, v_{0}=100$ : red and $\mu=4, v_{0}=40$ : blue). System density set to $\Phi=0.13$.

We then classified our system based on these criteria. The results of this classification for particles with self-propulsion speed $v_{0}=40$ are presented in Fig. 7.5. Here we see that higher magnetic moments $\mu$ allow chains to stay intact during reorientation (blue), even for increased ramping rates $\beta$. This observation is independent of the self-propulsion speed $v_{0}$ or the density of the system $\Phi$ (not shown).

Together with the results presented in section 7.2 , we conclude that in systems with time dependent external magnetic fields, temporal dynamics (assembly and 


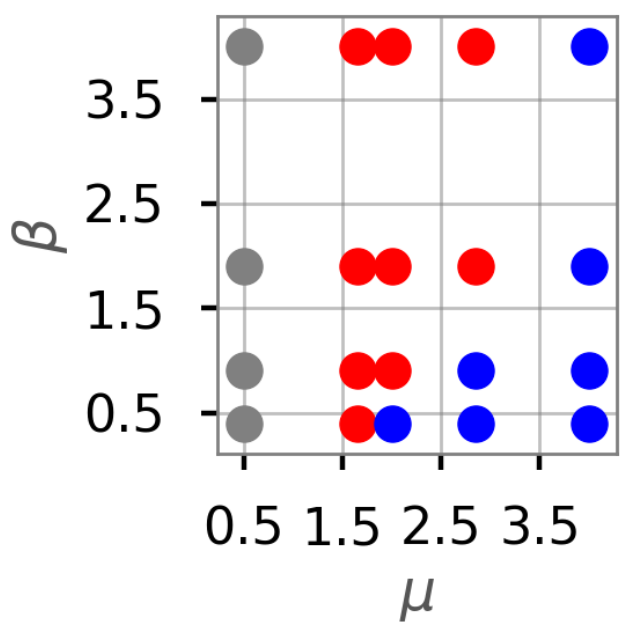

Figure 7.5.: Diagram showing three different scenarios when particle reorient in systems with time-dependent external magnetic field depending on magnetic moment $\mu$ and ramping rate of the external magnetic field $\beta$. Single particles reorient (gray), particles form chains that break when reorienting (red) and particles form chains that stay intact when reorienting (blue).

disassembly) are dictated by the external field but spatial organization (chain formation) is dictated by the strength of the magnetic moment and the resulting particle-particle interactions. Spatial organization of dipolar particles into chains and their elastic properties has been investigated in (rotating) external magnetic fields in other studies $44,66,67$. Here, the work focused on bending rigidity and the shape of bend chains in external magnetic fields, but not on the dynamical fragmentation and re-assembly of chains. With the magnetic field protocol we used, we demonstrated that the level of activity has little effect on the fragmentation dynamics in our systems. This result is in contrast to the results presented for systems with constant external magnetic field (see chapter 6), where both activity and density affect structure formation. 


\section{Conclusions}

In this thesis, we used Brownian dynamics simulations to study collective dynamics and self-assembly of dipolar active particles under various conditions. We addressed our main research questions in four parts.

First, we focused on structure formation in small systems, $N=36$, and studied the effect of confinement by applying different boundary conditions. Here, we mainly observed that particles self-assemble into two types of structures, chains and rings. In a diagram of states we showed what configurations are most likely to occur depending on the activity and magnetic moments of the particles. We observed that in systems without confinement, chains dominate the diagram of states, both for active as well as passive dipolar particles. For passive dipolar particles, analytical calculations 50 -53 predict rings to dominate for the investigated parameters for $N=36$ particles. These predictions seem to contradict our numerical observations at first. We have noticed that passive particles typically form chains of size $N \approx 3$ in our simulations. These chains can be considered as isolated sub-systems that interact on much longer time scales with other subsystems of similar size. These longer time scales are not sufficiently covered in our dilute simulations at $\Phi=0.01$. When we consider that the number of particles in each isolated sub-system is greatly reduced, both numerical simulations 
and analytical predictions result in chains as dominant structures in these smaller sub-systems. When we increased the density to $\Phi=0.03$ by reducing the box size we recovered the predicted ring configurations for passive particles. Other numerical studies have shown that much longer simulation times (up to a factor of 100) are needed to reach agreement between simulations and analytical predictions of structure formation in systems of passive dipolar particles [53]. However, due to their persistent motion, active particles explore their environment faster than passive particles and therefore reach long-term behavior at earlier times [139] which are covered in our simulations.

In systems with confinement, we observed that ring formation is promoted by the confinement. Calculation of a local structure order parameter revealed that rings dominantly form at walls in our simulations. This finding suggests that interactions with walls have an important influence on structure formation. We argue that two mechanisms are responsible for ring formation. One, walls reduce the degrees of freedom of chains of (active) dipolar particles. Therefore, already assembled chains can catch their own tail and form into a ring near a wall. And two, the increase of probability density at walls of the system drives particles to form larger clusters which favor ring configurations. The effect of boundary conditions on probability density fluxes of active particles has recently been investigated and demonstrates a method to manipulate trajectories and thus potentially affect the collective dynamics of active particles 85,87 .

While we observed that the geometry of the confinement affects structure formation to a greater extent, a recent study has demonstrated that reorienting torques at walls in numerical simulations are crucial to faithfully reproduce experimental observations of magnetotactic bacteria in circular traps [115. We note that the 


\section{Conclusions}

parameters chosen in that study are similar to the ones presented in this thesis.

Furthermore, we demonstrated that the probability of cluster formation in our system gives crucial insights to understand structure formation. Based on the probability of cluster formation, we showed that a critical magnetic strength $\mu^{*}$ can be defined. This critical magnetic strength increases linearly with the selfpropulsion speed of the particles and is independent of boundary conditions and density of the system. This finding suggest that activity can be treated as an effective temperature in this case. We used this interpretation to re-scale the magnetic moments of the active particles to an effective magnetic moment as function of self-propulsion speed.

We then rewrote a previously derived analytical expression for the expected ratio of chains to rings in passive systems of dipolar particles [53] as a function of this effective magnetic moment. Based on this analytical expression, we constructed a diagram of states predicting ring and chain formation that is qualitatively in good agreement with our numerical results. A quantitative mismatch indicates the limits of interpreting activity as an effective temperature. Calculation of an effective temperature has been shown to depend on the choice of observable for re-scaling and can be affected by density, collective phenomena and anisotropy 120, 122 125.

Second, we analyzed the collective behavior of active dipolar particles in larger systems. To this extent, we performed numerical simulations with $N=1156$ particles. Here, we observed distinct states depending on the magnetic moments and self-propulsion speed of the particles, but also on the density of the system. We characterized these states by calculating three order parameters and summarized our results in diagrams of states. Qualitatively, or results match well with the 
observations presented in a similar study by Liao et al. [73. In particular, we observed spontaneous global alignment of particles at high levels of activity at low density, similar to the Vicsek model [3], and motility induced phase separation in dense systems of apolar particles at high self-propulsion speed [117. However, our diagrams of states discriminate the observed states in more detail and extend the studied range of magnetic moments.

Third, motivated by the level of agreement with $[73$, we continued to investigate how an external constant magnetic field affects structure formation of active dipolar particles. Here, we summarize our three most important observations.

One, external magnetic fields suppress the formation of bands at low $(\Phi=$ $0.13)$ and intermediate $(\Phi=0.23)$ densities. Instead, oriented gas and chain states dominate. We showed that these two states are practically independent of the level of activity when a strong external magnetic field is present in systems at intermediate density. This observation is dramatically different from chain formation observed in systems without external magnetic fields, where activity plays a crucial role in collective phenomena [73, 77, 99]. In our model, activity is introduced as an active force acting in the direction of the magnetic moment of the particle. The external magnetic field introduces a preferred orientation of the particles on a global level and therefore may change how activity affects collective dynamics in systems of active dipolar particles. These observations suggest that details on how activity is introduced into the system, especially when coupled to orientational degrees of freedom, may be important to understand collective phenomena in systems out of equilibrium.

Two, we observed oscillating chains as a transient state towards band formation in our system. We suspect that these oscillations are caused by a buckling insta- 


\section{Conclusions}

bility. In addition, we propose that fragmentation of long chains due to buckling instabilities allows chains of different lengths to coexist in dilute systems of active dipolar particles with high magnetic moments. This mechanism needs to be investigated in more detail in future studies. In particular, a quantitative analysis that shows the relation between oscillations and flexure number of the system might aid to understand buckling instabilities in active polymers [134].

Three, we observed that active dipolar particles form columnar structures that are qualitatively similar to structures induced by external magnetic fields in ferrofluids $[57,62,63]$. A quantitative analysis showed that the number of lanes per column and the columnar spacing decreases rapidly with increasing magnetic field strength. Columnar spacing in ferrofluids has been reported to not depend on the external magnetic field strength [62, 63]. We recovered this behavior for strong external magnetic fields. However, we observed a strong dependence of the columnar spacing and number of lanes per column for weak external magnetic fields. Experimental details like the application of the external magnetic field, presence of hydrodynamic interactions, polydispersity and boundary conditions might influence this result. In a first step, we have shown here that activity does not affect separation between columns to a great extent. In future studies, the effect of density could be investigated in more detail and compared to 62].

Finally, we introduced a time dependent external magnetic field by modulating the external field with a triangular function. An analysis of the average orientation as a function of the external magnetic field resulted in hysteresis curves. We characterized these hysteresis curves by two points, the coercive force and the relative remnant magnetization and identified that activity affects these parameters only to little extent. In addition, we saw that three possible dynamic scenarios were re- 
alized, when the external magnetic field switches its sign. One, individual particles reorient in a U-turn. Two, previously formed chains break when reorienting and reorganize when aligned with the new direction of the external field. Three, chains stay intact when reorienting and adopt a C-like shape during that process. We classified our system based on these three categories. Classification showed that given the magnetic field protocol we used, activity has little effect on dynamics and structure formation in our systems. Dynamics (assembly and disassembly) are dictated by the external magnetic field. Spatial organization (chain formation) is dictated by the magnetic moment and the resulting particle-particle interactions. As demonstrated in other studies, different magnetic field protocols may lead to different observations $67,140,141$. Understanding the effect time dependent external magnetic fields have on dipolar particles is important for programmed structure formation in and out of equilibrium $33,34,142,144$.

In conclusion, we have presented and characterized the plethora of structural dynamics and self-organization in systems of active dipolar particles over multiple scales in this thesis; starting from individual chains and rings and ending in structure formation and emerging collective behavior. We have shown that dipolar interactions further add to the rich complex collective behavior in systems of active particles. The addition of (time dependent) external magnetic fields shows how the complexity of this system quickly increases. We have demonstrated that Brownian dynamics simulations are an important tool towards better understanding of the complex collective phenomena that arise in such systems. 


\section{A. Simulation details}

The following table gives an overview about the parameters used in our simulations. 


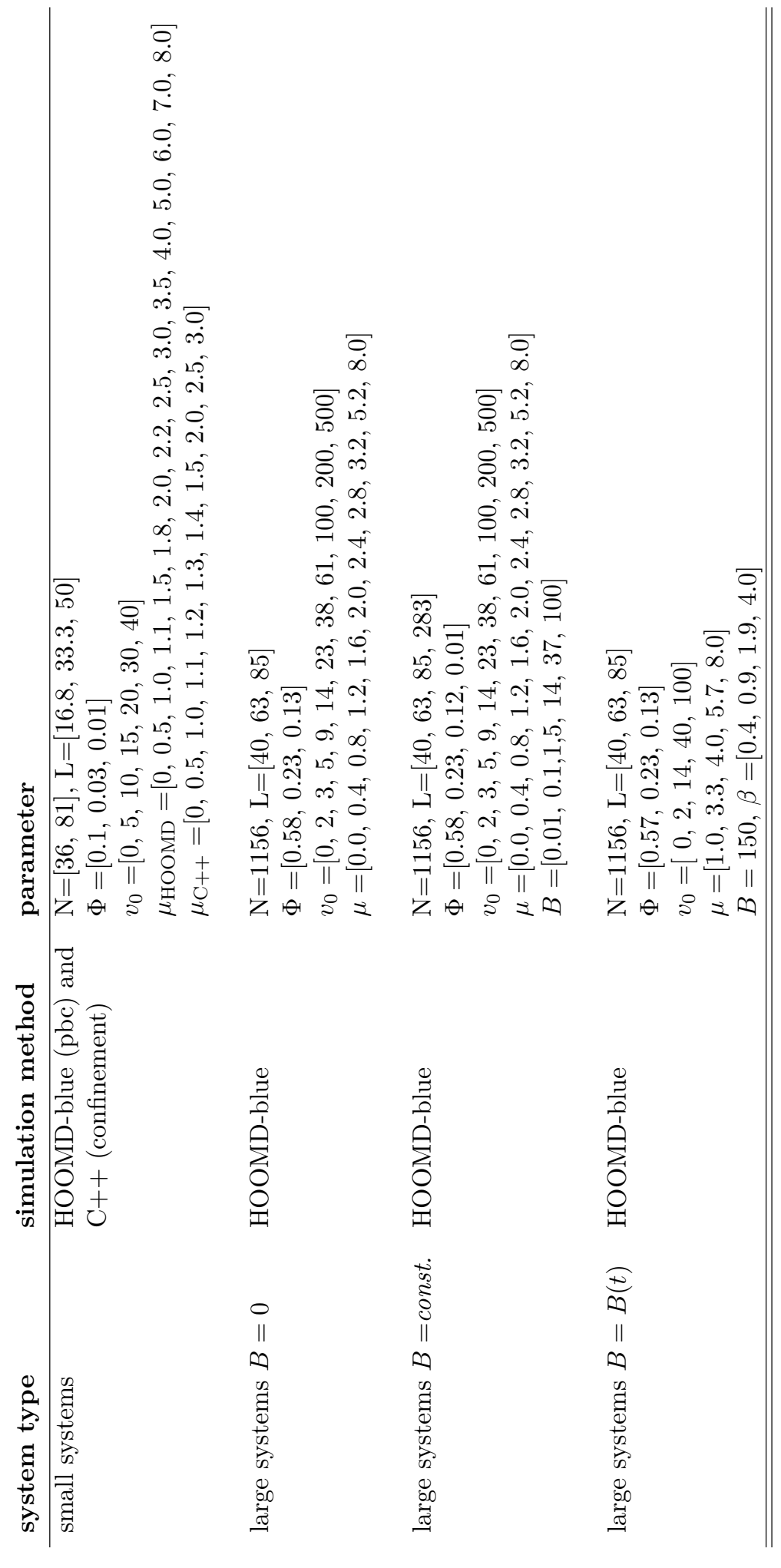





\section{Bibliography}

[1] P. Romanczuk, M. Bär, W. Ebeling, B. Lindner, and L. Schimansky-Geier, "Active Brownian particles: From individual to collective stochastic dynamics: From individual to collective stochastic dynamics", Eur. Phys. J. Spec. Top. 202, 1-162 (2012).

[2] A. Zöttl and H. Stark, "Emergent behavior in active colloids", J. Phys. Condens. Matter 28, 253001 (2016).

[3] T. Vicsek, A. Czirk, E. Ben-Jacob, I. Cohen, and O. Shochet, "Novel type of phase transition in a system of self-driven particles", Phys. Rev. Lett. 75, 1226-1229 (1995).

[4] H. Chaté, F. Ginelli, and R. Montagne, "Simple model for active nematics: Quasi-long-range order and giant fluctuations", Phys. Rev. Lett. 96, 180602 $(2006)$

[5] A. Baskaran and M. C. Marchetti, "Statistical mechanics and hydrodynamics of bacterial suspensions", Proc. Natl. Acad. Sci. U. S. A. 106, 15567$15572(2009)$

[6] J. Krause, G. D. Ruxton, G. Ruxton, I. G. Ruxton, et al., Living in groups (Oxford University Press, 2002). 


\section{Bibliography}

[7] I. D. Couzin, J. Krause, N. R. Franks, and S. A. Levin, "Effective leadership and decision-making in animal groups on the move", Nature 433, 513-516 $(2005)$

[8] D. Sumpter, J. Buhl, D. Biro, and I. Couzin, "Information transfer in moving animal groups", Theory Biosci. 127, 177-186 (2008).

[9] V. Guttal and I. D. Couzin, "Social interactions, information use, and the evolution of collective migration", Proc. Natl. Acad. Sci. U. S. A. 107, $16172-16177$ (2010)

[10] J. Tailleur and M. E. Cates, "Statistical mechanics of interacting run-andtumble bacteria", Phys. Rev. Lett. 100, 218103 (2008).

[11] T. Speck, J. Bialké, A. M. Menzel, and H. Löwen, "Effective cahn-hilliard equation for the phase separation of active brownian particles", Phys. Rev. Lett. 112, 218304 (2014).

[12] S. C. Takatori, W. Yan, and J. F. Brady, "Swim pressure: Stress generation in active matter", Phys. Rev. Lett. 113, 028103 (2014).

[13] T. F. Farage, P. Krinninger, and J. M. Brader, "Effective interactions in active Brownian suspensions", Phys. Rev. E - Stat. Nonlinear, Soft Matter Phys. 91, 042310 (2015).

[14] R. Dreyfus, J. Baudry, M. L. Roper, M. Fermigier, H. A. Stone, and J. Bibette, "Microscopic artificial swimmers", Nature 437, 862-865 (2005).

[15] G. P. Alexander and J. M. Yeomans, "Dumb-bell swimmers", EPL (Europhysics Lett. 83, 34006 (2008) 
[16] E. Lauga and T. R. Powers, "The hydrodynamics of swimming microorganisms", Reports Prog. Phys. 72, 096601 (2009).

[17] M. T. Downton and H. Stark, "Simulation of a model microswimmer", J. Phys. Condens. Matter 21, 204101 (2009).

[18] R. Brown, "XXVII. A brief account of microscopical observations made in the months of June, July and August 1827, on the particles contained in the pollen of plants; and on the general existence of active molecules in organic and inorganic bodies ", Philos. Mag. 4, 161-173 (1828).

[19] A. Einstein, "Über die von der molekularkinetischen Theorie der Wärme geforderte Bewegung von in ruhenden Flüssigkeiten suspendierten Teilchen", Ann. Phys. 322, 549-560 (1905).

[20] E. M. Purcell, "Life at low Reynolds number", Am. J. Phys. 45, 3-11 (1977).

[21] K. E. Peyer, L. Zhang, and B. J. Nelson, "Bio-inspired magnetic swimming microrobots for biomedical applications", Nanoscale 5, 1259-1272 (2013).

[22] K. Bente, A. Codutti, F. Bachmann, and D. Faivre, "Biohybrid and Bioinspired Magnetic Microswimmers", Small 14, 1704374 (2018).

[23] D. A. Bazylinski and R. B. Frankel, "Magnetosome formation in prokaryotes", Nat. Rev. Microbiol. 2, 217-230 (2004).

[24] D. Faivre and D. Schüler, "Magnetotactic bacteria and magnetosomes", Chem. Rev. 108, 4875-4898 (2008).

[25] R. B. Frankel and D. A. Bazylinski, "Magnetosomes and magneto-aerotaxis", in Contrib. microbiol. Vol. 16, edited by M. Collin and R. Schuch (Karger, Basel, 2009), pp. 182-193. 


\section{Bibliography}

[26] C. T. Lefèvre, M. Bennet, L. Landau, P. Vach, D. Pignol, D. A. Bazylinski, R. B. Frankel, S. Klumpp, and D. Faivre, "Diversity of magneto-aerotactic behaviors and oxygen sensing mechanisms in cultured magnetotactic bacteria", Biophys. J. 107, 527-538 (2014).

[27] S. Klumpp and D. Faivre, "Magnetotactic bacteria: Magnetic navigation on the microscale", Eur. Phys. J. Spec. Top. 225, 2173-2188 (2016).

[28] S. Klumpp, C. T. Lefèvre, M. Bennet, and D. Faivre, "Swimming with magnets: From biological organisms to synthetic devices", Phys. Rep. 789, 1-54 $(2019)$

[29] O. Felfoul, M. Mohammadi, S. Taherkhani, D. De Lanauze, Y. Zhong Xu, D. Loghin, S. Essa, S. Jancik, D. Houle, M. Lafleur, L. Gaboury, M. Tabrizian, N. Kaou, M. Atkin, T. Vuong, G. Batist, N. Beauchemin, D. Radzioch, and S. Martel, "Magneto-aerotactic bacteria deliver drug-containing nanoliposomes to tumour hypoxic regions", Nat. Nanotechnol. 11, 941-947 (2016).

[30] M. M. Stanton, B. W. Park, D. Vilela, K. Bente, D. Faivre, M. Sitti, and S. Sánchez, "Magnetotactic Bacteria Powered Biohybrids Target E. coli Biofilms", ACS Nano 11, 9968-9978 (2017).

[31] L. Zhang, J. J. Abbott, L. Dong, B. E. Kratochvil, D. Bell, and B. J. Nelson, "Artificial bacterial flagella: Fabrication and magnetic control", Appl. Phys. Lett. 94, 64107 (2009).

[32] D. Nishiguchi, J. Iwasawa, H. R. Jiang, and M. Sano, "Flagellar dynamics of chains of active Janus particles fueled by an AC electric field", New J. Phys. 20, 015002 (2018). 
[33] J. Yan, S. C. Bae, and S. Granick, "Colloidal superstructures programmed into magnetic janus particles", Adv. Mater. 27, 874-879 (2015).

[34] G. Steinbach, M. Schreiber, D. Nissen, M. Albrecht, E. Novak, P. A. Sánchez, S. S. Kantorovich, S. Gemming, and A. Erbe, "Field-responsive colloidal assemblies defined by magnetic anisotropy", Phys. Rev. E 100, 012608 (2019).

[35] A. Walther and A. H. Müller, "Janus particles", Soft Matter 4, 663-668 $(2008)$

[36] X. Ma and S. Sanchez, "A bio-catalytically driven Janus mesoporous silica cluster motor with magnetic guidance", Chem. Commun. 51, 5467-5470 $(2015)$,

[37] L. Baraban, R. Streubel, D. Makarov, L. Han, D. Karnaushenko, O. G. Schmidt, and G. Cuniberti, "Fuel-free locomotion of janus motors: Magnetically induced thermophoresis", ACS Nano 7, 1360-1367 (2013).

[38] C. Holm and J.-J. J. Weis, "The structure of ferrofluids: A status report", Curr. Opin. Colloid Interface Sci. 10, 133-140 (2005).

[39] J. J. Weis and D. Levesque, "Simple dipolar fluids as generic models for soft matter", Adv. Polym. Sci. 185, 163-225 (2005).

[40] S. H. Klapp, "Collective dynamics of dipolar and multipolar colloids: From passive to active systems", Curr. Opin. Colloid Interface Sci. 21, 76-85 $(2016)$.

[41] P. G. de Gennes and P. A. Pincus, "Pair correlations in a ferromagnetic colloid", Phys. der Kondens. Mater. 11, 189-198 (1970). 


\section{Bibliography}

[42] I. S. Jacobs and C. P. Bean, "An approach to elongated fine-particle magnets", Phys. Rev. 100, 1060-1067 (1955).

[43] R. Messina, L. A. Khalil, and I. Stanković, "Self-assembly of magnetic balls: From chains to tubes", Phys. Rev. E - Stat. Nonlinear, Soft Matter Phys. 89, $011202(2014)$.

[44] B. Kiani, D. Faivre, and S. Klumpp, "Elastic properties of magnetosome chains", New J. Phys. 17, 43007 (2015).

[45] M. E. Van Leeuwen and B. Smit, "What makes a polar liquid a liquid?", Phys. Rev. Lett. 71, 3991-3994 (1993).

[46] G. T. Gao, X. C. Zeng, and W. Wang, "Vapor-liquid coexistence of quasitwo-dimensional Stockmayer fluids", J. Chem. Phys. 106, 3311-3317 (1997).

[47] J. J. Weis, "Preliminary communication orientational structure of quasi-twodimensional dipolar hard spheres", Mol. Phys. 93, 361-364 (1998).

[48] P. I. Teixeira, J. M. Tavares, and M. M. Telo Da Gama, "Effect of dipolar forces on the structure and thermodynamics of classical fluids", J. Phys. Condens. Matter 12, R411-R434 (2000).

[49] K. Butter, P. H. Bomans, P. M. Frederik, G. J. Vroege, and A. P. Philipse, "Direct observation of dipolar chains in ferrofluids in zero field using cryogenic electron microscopy", J. Phys. Condens. Matter 15, S1451 (2003).

[50] J. J. Weis and D. Levesque, "Chain formation in low density dipolar hard spheres: A Monte Carlo study", Phys. Rev. Lett. 71, 2729-2732 (1993). 
[51] T. A. Prokopieva, V. A. Danilov, S. S. Kantorovich, and C. Holm, "Ground state structures in ferrofluid monolayers", Phys. Rev. E - Stat. Nonlinear, Soft Matter Phys. 80, 031404 (2009).

[52] S. S. Kantorovich, A. O. Ivanov, L. Rovigatti, J. M. Tavares, and F. Sciortino, "Temperature-induced structural transitions in self-assembling magnetic nanocolloids", Phys. Chem. Chem. Phys. 17, 16601-16608 (2015).

[53] S. Kantorovich, A. O. Ivanov, L. Rovigatti, J. M. Tavares, and F. Sciortino, "Nonmonotonic magnetic susceptibility of dipolar hard-spheres at low temperature and density", Phys. Rev. Lett. 110, 148306 (2013).

[54] R. E. Goldstein, D. P. Jackson, and S. A. Langer, "Dynamics of pattern formation in magnetic fluids", Colloids Surfaces A Physicochem. Eng. Asp. 80, 29-37 (1993).

[55] A. J. Dickstein, S. Erramilli, R. E. Goldstein, D. P. Jackson, and S. A. Langer, "Labyrinthine pattern formation in magnetic fluids", Science 261, 1012-1015 (1993).

[56] F. Elias, C. Flament, J. C. Bacri, and S. Neveu, "Macro-organized patterns in ferrofluid layer: experimental studies", J. Phys. II 7, 711-728 (1997).

[57] G. A. Flores, J. Liu, M. Mohebi, and N. Jamasbi, "Magnetic-field-induced nonequilibrium structures in a ferrofluid emulsion", Phys. Rev. E - Stat. Physics, Plasmas, Fluids, Relat. Interdiscip. Top. 59, 751-762 (1999).

[58] D. J. Klingenberg, "Magnetorheology: Applications and challenges", AIChE J. 47, 246-249 (2001). 


\section{Bibliography}

[59] M. J. Stevens and G. S. Grest, "Coexistence in dipolar fluids in a field", Phys. Rev. Lett. 72, 3686-3689 (1994).

[60] M. J. Stevens and G. S. Grest, "Structure of soft-sphere dipolar fluids", Phys. Rev. E 51, 5962-5975 (1995).

[61] Z. Wang, C. Holm, and H. W. Müller, "Molecular dynamics study on the equilibrium magnetization properties and structure of ferrofluids", Phys. Rev. E - Stat. Physics, Plasmas, Fluids, Relat. Interdiscip. Top. 66, 021405 $(2002)$,

[62] J. Liu, E. M. Lawrence, A. Wu, M. L. Ivey, G. A. Flores, K. Javier, J. Bibette, and J. Richard, "Field-induced structures in ferrofluid emulsions", Phys. Rev. Lett. 74, 2828 (1995).

[63] M. Ivey, J. Liu, Y. Zhu, and S. Cutillas, "Magnetic-field-induced structural transitions in a ferrofluid emulsion", Phys. Rev. E - Stat. Nonlinear, Soft Matter Phys. 63, 011403 (2001).

[64] E. Climent, M. R. Maxey, and G. E. Karniadakis, "Dynamics of Self-Assembled Chaining in Magnetorheological Fluids", Langmuir 20, 507-513 (2004).

[65] J. De Vicente, D. J. Klingenberg, and R. Hidalgo-Alvarez, "Magnetorheological fluids: A review", Soft Matter 7, 3701-3710 (2011).

[66] S. Melle and J. E. Martin, "Chain model of a magnetorheological suspension in a rotating field", J. Chem. Phys. 118, 9875-9881 (2003).

[67] R. Patel and B. Chudasama, "Hydrodynamics of chains in ferrofluid-based magnetorheological fluids under rotating magnetic field", Phys. Rev. E Stat. Nonlinear, Soft Matter Phys. 80, 012401 (2009). 
[68] D. C. Jiles and D. L. Atherton, "Theory of ferromagnetic hysteresis", J. Magn. Magn. Mater. 61, 48-60 (1986).

[69] M. Kroell, M. Pridoehl, G. Zimmermann, L. Pop, S. Odenbach, and A. Hartwig, "Magnetic and rheological characterization of novel ferrofluids", J. Magn. Magn. Mater. 289, 21-24 (2005).

[70] A. Kaiser, K. Popowa, and H. Löwen, "Active dipole clusters: From helical motion to fission", Phys. Rev. E - Stat. Nonlinear, Soft Matter Phys. 92, $012301(2015)$.

[71] F. Guzmán-Lastra, A. Kaiser, and H. Löwen, "Fission and fusion scenarios for magnetic microswimmer clusters", Nat. Commun. 7, 13519 (2016).

[72] A. Martín-Gómez, D. Levis, A. Díaz-Guilera, and I. Pagonabarraga, "Collective motion of active Brownian particles with polar alignment", Soft Matter 14, 2610-2618 (2018).

[73] G. J. Liao, C. K. Hall, and S. H. Klapp, "Dynamical self-assembly of dipolar active Brownian particles in two dimensions", Soft Matter 16, 2208-2223 $(2020)$

[74] I. R. Bruss and S. C. Glotzer, "Phase separation of self-propelled ballistic particles", Phys. Rev. E 97, 042609 (2018).

[75] I. R. Bruss and S. C. Glotzer, "Curvature-induced microswarming", Soft Matter 13, 5117-5121 (2017).

[76] M. Sanoria, R. Chelakkot, and A. Nandi, "Influence of interaction softness on phase separation of active particles", Phys. Rev. E 103, 052605 (2021). 


\section{Bibliography}

[77] R. C. Maloney, G. J. Liao, S. H. Klapp, and C. K. Hall, "Clustering and phase separation in mixtures of dipolar and active particles", Soft Matter 16, 3779-3791 (2020).

[78] R. C. Maloney and C. K. Hall, "Clustering and Phase Separation in Mixtures of Dipolar and Active Particles in an External Field", Langmuir 36, 6378$6387(2020)$.

[79] J. Toner, Y. Tu, and S. Ramaswamy, "Hydrodynamics and phases of flocks", Ann. Phys. (N. Y). 318, 170-244 (2005).

[80] A. P. Berke, L. Turner, H. C. Berg, and E. Lauga, "Hydrodynamic attraction of swimming microorganisms by surfaces", Phys. Rev. Lett. 101, 038102 $(2008)$

[81] M. B. Wan, C. J. Olson Reichhardt, Z. Nussinov, and C. Reichhardt, "Rectification of swimming bacteria and self-driven particle systems by arrays of asymmetric barriers", Phys. Rev. Lett. 101, 18102 (2008).

[82] G. Li and J. X. Tang, "Accumulation of microswimmers near a surface mediated by collision and rotational Brownian motion", Phys. Rev. Lett. 103, 078101 (2009).

[83] M. C. Marchetti, J. F. Joanny, S. Ramaswamy, T. B. Liverpool, J. Prost, M. Rao, and R. A. Simha, "Hydrodynamics of soft active matter", Rev. Mod. Phys. 85, 1143-1189 (2013).

[84] K. Drescher, "2.2 Bacterial Flow Field", in Microswimmers - from single particle motion to collective behaviour, edited by G. Gompper, C. Bechinger, S. Herminghaus, R. Isele-Holder, U. B. Kaupp, H. Löwen, H. Stark, and 
R. G. Winkler (Forschungszentrum Jülich GmbH Zentralbibliothek, Jülich, 2015) Chap. Biological Microswimmers, B2.5-B2.6.

[85] J. Cammann, F. J. Schwarzendahl, T. Ostapenko, D. Lavrentovich, O. Bäumchen, and M. G. Mazza, "Emergent probability fluxes in confined microbial navigation", Proc. Natl. Acad. Sci. 118, e2024752118 (2021).

[86] J. P. Hernandez-Ortiz, P. T. Underhill, and M. D. Graham, "Dynamics of confined suspensions of swimming particles", J. Phys. Condens. Matter 21, 204107 (2009).

[87] T. Ostapenko, F. J. Schwarzendahl, T. J. Böddeker, C. T. Kreis, J. Cammann, M. G. Mazza, and O. Bäumchen, "Curvature-guided motility of microalgae in geometric confinement", Phys. Rev. Lett. 120, 68002 (2018).

[88] P. Sartori, E. Chiarello, G. Jayaswal, M. Pierno, G. Mistura, P. Brun, A. Tiribocchi, and E. Orlandini, "Wall accumulation of bacteria with different motility patterns", Phys. Rev. E 97, 022610 (2018).

[89] D. Chandler, J. D. Weeks, and H. C. Andersen, "Van der waals picture of liquids, solids, and phase transformations", Science (80-. ). 220, 787-794 (1983)

[90] K. W. Yung, P. B. Landecker, and D. D. Villani, "An Analytic Solution for the Force Between Two Magnetic Dipoles", Magn. Electr. Sep. 9, 39-52 (1998).

[91] K. W. Yung, P. B. Landecker, and D. D. Villani, "An Analytic Solution for the Torque Between Two Magnetic Dipoles", Magn. Electr. Sep. 10, 29-33 (1999) 


\section{Bibliography}

[92] G. Volpe, I. Buttinoni, D. Vogt, H. J. Kümmerer, and C. Bechinger, "Microswimmers in patterned environments", Soft Matter 7, 8810-8815 (2011).

[93] V. Kantsler, J. Dunkel, M. Polin, and R. E. Goldstein, "Ciliary contact interactions dominate surface scattering of swimming eukaryotes", Proc. Natl. Acad. Sci. U. S. A. 110, 1187-1192 (2013).

[94] C. Bechinger, R. Di Leonardo, H. Löwen, C. Reichhardt, G. Volpe, and G. Volpe, "Active particles in complex and crowded environments", Rev. Mod. Phys. 88, 45006 (2016).

[95] D. K. Barupal and O. Fiehn, "Generating the blood exposome database using a comprehensive text mining and database fusion approach", Environ. Health Perspect. 127, 2825-2830 (2019).

[96] S. S. Das, A. P. Andrews, and S. C. Greer, "Living poly ( $\alpha$-methylstyrene) near the polymerization line. IV. Extent of polymerization as a function of temperature", J. Chem. Phys. 102, 2951-2959 (1995).

[97] J. Stambaugh, K. Van Workum, J. F. Douglas, and W. Losert, "Polymerization transitions in two-dimensional systems of dipolar spheres", Phys. Rev. E - Stat. Nonlinear, Soft Matter Phys. 72, 031301 (2005).

[98] H. Schmidle, C. K. Hall, O. D. Velev, and S. H. Klapp, "Phase diagram of two-dimensional systems of dipole-like colloids", Soft Matter 8, 1521-1531 $(2012)$

[99] V. Telezki and S. Klumpp, "Simulations of structure formation by confined dipolar active particles", Soft Matter 16, 10537-10547 (2020).

[100] GUROBI OPTIMIZATION INC., Gurobi optimizer reference manual, 2021. 
[101] J. P. Bouchaud and A. Georges, "Anomalous diffusion in disordered media: Statistical mechanisms, models and physical applications", Phys. Rep. 195, $127-293$ (1990).

[102] R. Metzler and J. Klafter, "The random walk's guide to anomalous diffusion: A fractional dynamics approach", Phys. Rep. 339, 1-77 (2000).

[103] P. Virtanen et al., "SciPy 1.0: fundamental algorithms for scientific computing in Python", Nat. Methods 17, 261-272 (2020).

[104] V. Calandrini, E. Pellegrini, P. Calligari, K. Hinsen, and G. Kneller, "nMoldyn - Interfacing spectroscopic experiments, molecular dynamics simulations and models for time correlation functions", École thématique la Société Française la Neutron. 12, 201-232 (2011).

[105] S. H. L. Klapp, "Dipolar fluids under external perturbations", J. Phys. Condens. Matter 17, R525-R550 (2005).

[106] C. H. Rycroft, "VORO++: A three-dimensional Voronoi cell library in C++", Chaos 19, 41111 (2009).

[107] V. Ramasubramani, B. D. Dice, E. S. Harper, M. P. Spellings, J. A. Anderson, and S. C. Glotzer, "freud: A software suite for high throughput analysis of particle simulation data", Comput. Phys. Commun. 254, 107275 (2020).

[108] R. L. Honeycutt, "Stochastic Runge-Kutta algorithms. I. White noise", Phys. Rev. A 45, 600-603 (1992).

[109] P. E. Kloeden and E. Platen, "Higher-order implicit strong numerical schemes for stochastic differential equations", J. Stat. Phys. 66, 283-314 (1992). 


\section{Bibliography}

[110] M. Matsumoto and T. Nishimura, "Mersenne Twister: A 623-Dimensionally Equidistributed Uniform Pseudo-Random Number Generator", ACM Trans. Model. Comput. Simul. 8, 3-30 (1998).

[111] J. A. Anderson, C. D. Lorenz, and A. Travesset, "General purpose molecular dynamics simulations fully implemented on graphics processing units", J. Comput. Phys. 227, 5342-5359 (2008).

[112] J. A. Anderson, J. Glaser, and S. C. Glotzer, "HOOMD-blue: A Python package for high-performance molecular dynamics and hard particle Monte Carlo simulations", Comput. Mater. Sci. 173, 109363 (2020).

[113] C. S. Adorf, P. M. Dodd, V. Ramasubramani, and S. C. Glotzer, "Simple data and workflow management with the signac framework", Comput. Mater. Sci. 146, 220-229 (2018).

[114] V. Ramasubramani, C. Adorf, P. Dodd, B. Dice, and S. Glotzer, "signac: A Python framework for data and workflow management", in Proc. 17th python sci. conf. Edited by F. Akici, D. Lippa, D. Niederhut, and M. Pacer (2018), pp. 152-159.

[115] A. Codutti, M. A. Charsooghi, E. Cerdá-Doñate, H. M. Taïeb, T. Robinson, D. Faivre, and S. Klumpp, "Single-cell motion of magnetotactic bacteria in microfluidic confinement: interplay between surface interaction and magnetic torque", bioRxiv, 2021.03.27.437322 (2021)

[116] I. Buttinoni, J. Bialké, F. Kümmel, H. Löwen, C. Bechinger, and T. Speck, "Dynamical clustering and phase separation in suspensions of self-propelled colloidal particles", Phys. Rev. Lett. 110, 238301 (2013). 
[117] M. E. Cates and J. Tailleur, "Motility-induced phase separation", Annu. Rev. Condens. Matter Phys. 6, 219-244 (2015).

[118] P. Digregorio, D. Levis, A. Suma, L. F. Cugliandolo, G. Gonnella, and I. Pagonabarraga, "Full Phase Diagram of Active Brownian Disks: From Melting to Motility-Induced Phase Separation", Phys. Rev. Lett. 121, 098003 $(2018)$,

[119] D. Loi, S. Mossa, and L. F. Cugliandolo, "Effective temperature of active matter", Phys. Rev. E - Stat. Nonlinear, Soft Matter Phys. 77, 051111 $(2008)$.

[120] S. Smyk, V. Telezki, J. Riepl, J. Hayes, and S. Klumpp, "Orientation fluctuations in magnetotactic swimming", Eur. Phys. J. Spec. Top. 230, 1099$1103(2021)$

[121] P. G. de Gennes, "Conformations of Polymers Attached to an Interface", Macromolecules 13, 1069-1075 (1980).

[122] A. Suma, G. Gonnella, G. Laghezza, A. Lamura, A. Mossa, and L. F. Cugliandolo, "Dynamics of a homogeneous active dumbbell system", Phys. Rev. E - Stat. Nonlinear, Soft Matter Phys. 90, 052130 (2014).

[123] D. Levis and L. Berthier, "From single-particle to collective effective temperatures in an active fluid of self-propelled particles", Epl 111, 60006 (2015).

[124] Z. Preisler and M. Dijkstra, "Configurational entropy and effective temperature in systems of active Brownian particles", Soft Matter 12, 6043-6048 (2016). 


\section{Bibliography}

[125] L. F. Cugliandolo, G. Gonnella, and I. Petrelli, "Effective Temperature in Active Brownian Particles", Fluct. Noise Lett. 18, 1940008 (2019).

[126] J. M. Tavares, J. J. Weis, and M. M. Telo da Gama, "Quasi-two-dimensional dipolar fluid at low densities: Monte Carlo simulations and theory", Phys. Rev. E - Stat. Physics, Plasmas, Fluids, Relat. Interdiscip. Top. 65, 061201 $(2002)$.

[127] V. Schaller, C. Weber, C. Semmrich, E. Frey, and A. R. Bausch, "Polar patterns of driven filaments", Nature 467, 73-77 (2010).

[128] A. Jayaram, A. Fischer, and T. Speck, "From scalar to polar active matter: Connecting simulations with mean-field theory", Phys. Rev. E 101, 022602 $(2020)$.

[129] M. Mohebi, N. Jamasbi, and J. Liu, "Simulation of the formation of nonequilibrium structures in magnetorheological fluids subject to an external magnetic field", Phys. Rev. E - Stat. Physics, Plasmas, Fluids, Relat. Interdiscip. Top. 54, 5407-5413 (1996).

[130] J. J. Weis, "Simulation of quasi-two-dimensional dipolar systems", J. Phys. Condens. Matter 15, S1471 (2003)

[131] J. Blaschke, M. Maurer, K. Menon, A. Zöttl, and H. Stark, "Phase separation and coexistence of hydrodynamically interacting microswimmers", Soft Matter 12, 9821-9831 (2016).

[132] E. Sesé-Sansa, I. Pagonabarraga, and D. Levis, "Velocity alignment promotes motility-induced phase separation", Epl 124, 30004 (2018). 
[133] P. Langevin, "Sur la théorie du magnétisme", J. Phys. Theor. Appl. 4, 678$693(1905)$.

[134] K. R. Prathyusha, S. Henkes, and R. Sknepnek, "Dynamically generated patterns in dense suspensions of active filaments", Phys. Rev. E 97, 022606 (2018),

[135] S. Melle, O. G. Calderón, M. A. Rubio, and G. G. Fuller, "Microstructure evolution in magnetorheological suspensions governed by Mason number", Phys. Rev. E - Stat. Physics, Plasmas, Fluids, Relat. Interdiscip. Top. 68, 041503 (2003).

[136] H. Chaté, F. Ginelli, G. Grégoire, and F. Raynaud, "Collective motion of self-propelled particles interacting without cohesion", Phys. Rev. E - Stat. Nonlinear, Soft Matter Phys. 77, 046113 (2008).

[137] A. Raghunathan, Y. Melikhov, J. E. Snyder, and D. C. Jiles, "Modeling the temperature dependence of hysteresis based on jiles-atherton theory", IEEE Trans. Magn. 45, 3954-3957 (2009).

[138] A. Raghunathan, Y. Melikhov, J. E. Snyder, and D. C. Jiles, "Theoretical model of temperature dependence of hysteresis based on mean field theory", IEEE Trans. Magn. 46, 1507-1510 (2010).

[139] M. Zeitz, K. Wolff, and H. Stark, "Active Brownian particles moving in a random Lorentz gas", Eur. Phys. J. E 40, 10.1140/epje/i2017-11510-0 $(2017)$.

[140] S. Jäger and S. H. L. Klapp, "Pattern formation of dipolar colloids in rotating fields: layering and synchronization", Soft Matter 7, 6606 (2011). 


\section{Bibliography}

[141] A. Cēbers and I. Javaitis, "Dynamics of a flexible magnetic chain in a rotating magnetic field", Phys. Rev. E 69, 21404 (2004).

[142] J. Yan, M. Bloom, S. C. Bae, E. Luijten, and S. Granick, "Linking synchronization to self-assembly using magnetic Janus colloids", Nature 491, $578-581(2012)$

[143] S. Jäger, H. Stark, and S. H. Klapp, "Dynamics of cluster formation in driven magnetic colloids dispersed on a monolayer", J. Phys. Condens. Matter 25, 195104 (2013).

[144] J. E. Martin and A. Snezhko, "Driving self-assembly and emergent dynamics in colloidal suspensions by time-dependent magnetic fields", Reports Prog. Phys. 76, 126601 (2013). 


\section{Acknowledgments}

I want to start by thanking my supervisor Stefan Klumpp, not only for the opportunity to pursue a doctorate in his group, but also for the countless discussions we had during my thesis period. I value your feedback and your guidance and sincerely appreciate the fact that you always managed to take time out of your busy schedule. Please accept my deepest thanks. I would also like to thank all current and former group members of the Theoretical Biophysics group. Special thanks go to the following people. Leila Abbaspour, who started her Ph.D. around the same time as I did. Together we maneuvered not only through research challenges but also helped each other when encountered with other difficulties. Sarah Mohammadinejad, Omar Munoz and Konrad Marx who worked on similar topics and thus made technical discussions about shared challenges possible. Regina Wunderlich and Dana Sachs for their administrative work. Thank you Damien Faivre, Agnese Codutti and Mohammad Charsooghi for helpful and engaging discussions about magnetotactic bacteria. In addition, I want to thank Julius Pätzold for his help with the implementation of the shortest path algorithm. Last, but not least, I want to thank the members of my thesis committee Karen Alim and Marcus Müller for their incredibly helpful comments and discussions during our meetings.

This work was supported by the Deutsche Forschungsgemeinschaft DFG (grant number KL818/2-2) within the priority program on Microswimmers (SPP 1726). 